\title{
HTGR Steam Generator Modeling
}

T. W. Kerlin

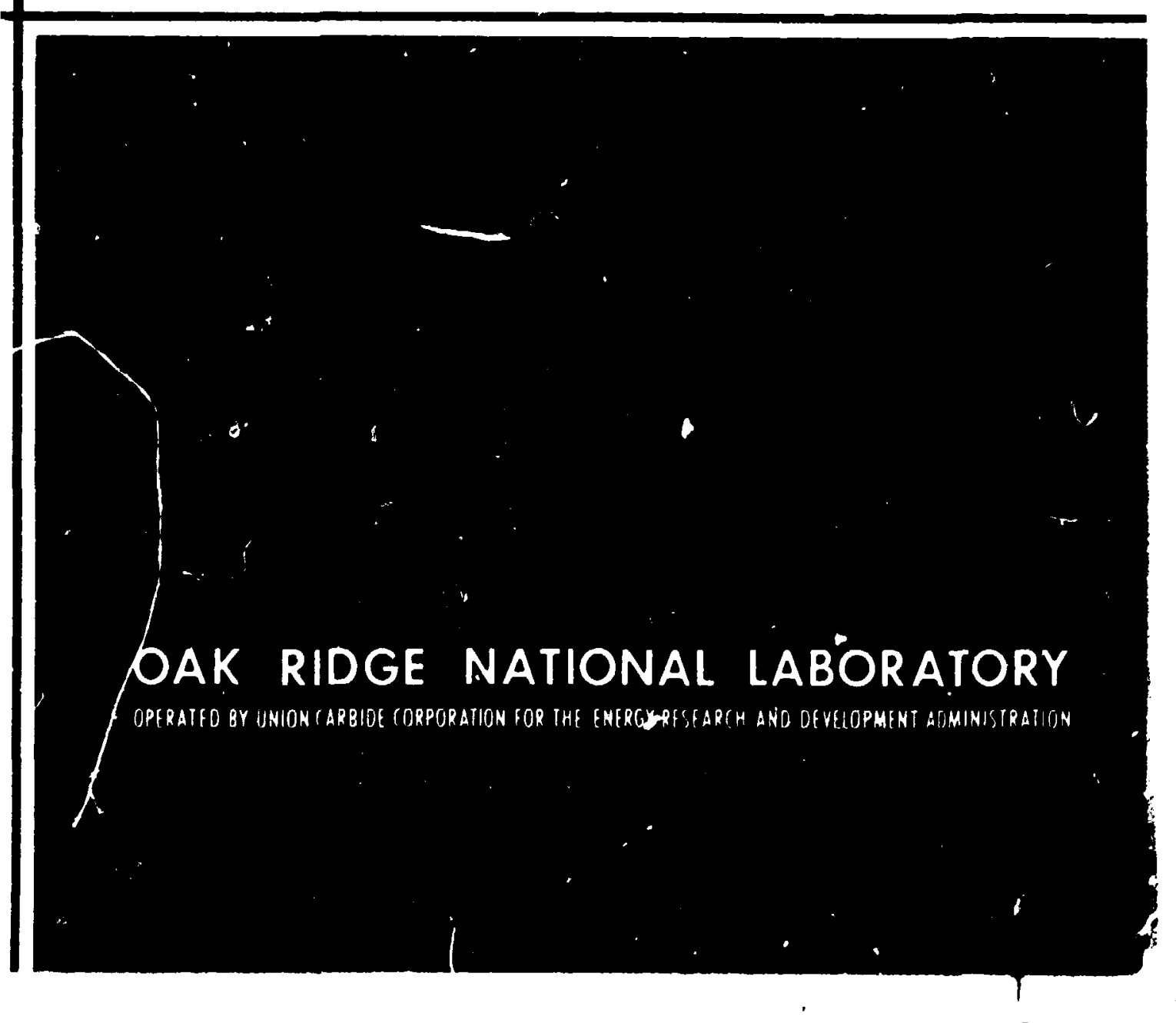




\begin{tabular}{|c|}
\hline 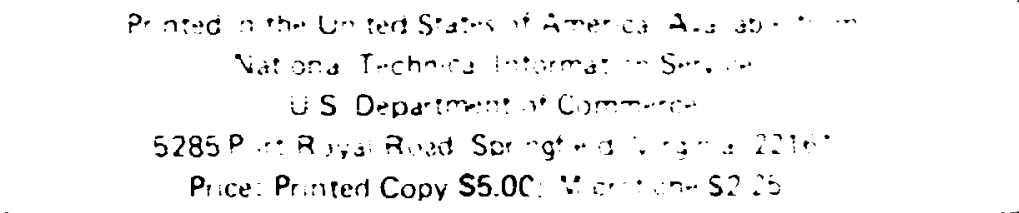 \\
\hline 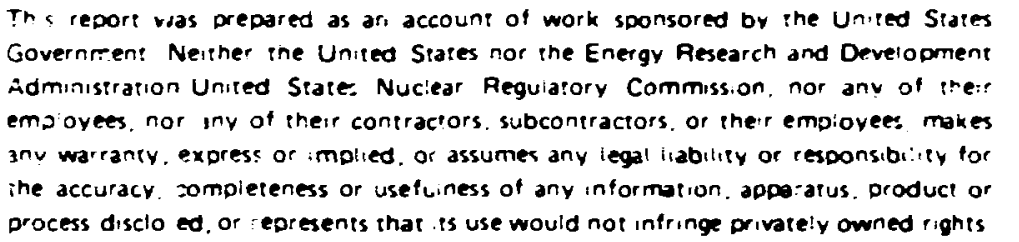 \\
\hline
\end{tabular}


Contract ino. H-7405-eng-26

HTGR STEAM GENERATOR :MDELING

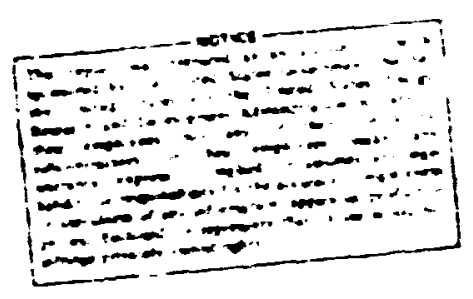

T. Kerlin, Director

\author{
This work was performed under subcontract 4122 by the \\ IJNIVERSITY OF TENMESSEE \\ Nuclear Eng; rieering Department \\ Knoxville, Tennessee 37916
Manuscript Completed - 6-2-i6
De:e Piblished - Jily. 1976
Prepared for the U.S. Nuclear Regulatory Commission Office :i Nuclear Reactor Regulation Under Interagency Agreement EROA 40-545

MOICE: This document contains information of a preliminary nature and was prepared primarily for internal use at the Oak Ridge National Laboratory. It is subject to revision or correction and therefore does not represent a final report.
OAK RIDGE NATIONAL LABORATORY
Oak Ridge, Tennessee 37830
operated by
UNION CARBIDE CORPORATION
for the

ENERGY RESEARCH AND OEVELOPME:IT ADMINISTRATION 
CORTENTS

Page

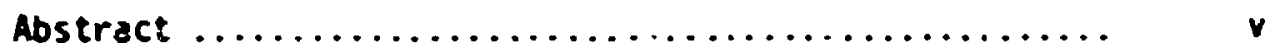

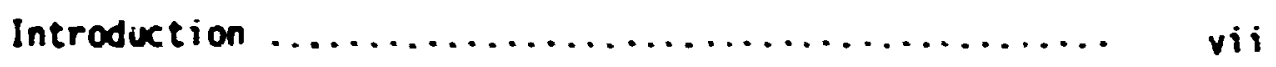

1. Fort St. Yrain Steam Generator Modeling by 5.1 . Chang $\ldots \ldots \ldots \ldots \ldots \ldots \ldots \ldots \ldots \ldots ., 1$

1.I A Descridiotion of the System .............. ,

1.2 Lumped Paraneter Analys is $\ldots \ldots \ldots \ldots \ldots \ldots \ldots .6$

1.3 Model Formulation $\ldots \ldots \ldots \ldots \ldots \ldots \ldots \ldots \ldots ., 7$

1.4 Mathematical Model $\ldots \ldots \ldots \ldots \ldots \ldots \ldots \ldots \ldots .8$

1.5 Linearization of Equations ............. 18

1.6 Results and Discussion ............... 32

References $\ldots \ldots \ldots \ldots \ldots \ldots \ldots \ldots \ldots \ldots \ldots \ldots . \ldots \ldots$

11. Nonlinear Modeling of a HTG Stean Generator
by Ming Huei Lee ...................... 51

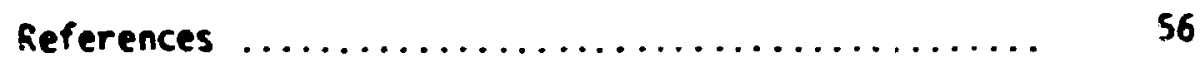

1II. Gas-Cooled Stean Generz cor Linear Model

by Oavid 6 . Renfro .................. 58

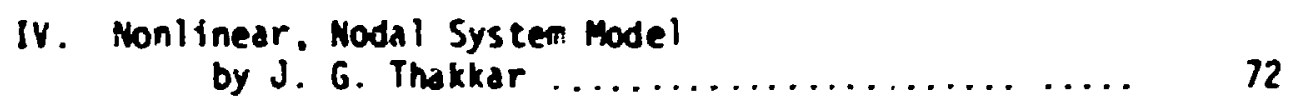

IV.l Steam Properties ................. 72

IV.2 Reactor $\ldots \ldots \ldots \ldots \ldots \ldots \ldots \ldots \ldots \ldots \ldots \ldots \ldots \ldots \ldots \ldots$

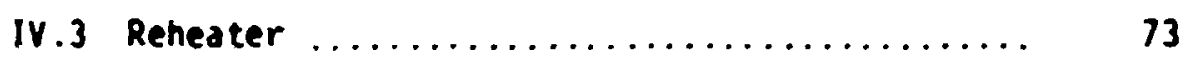

IV.4 Vielium Circulator .................. 74

IV.s Future dork $\ldots \ldots \ldots \ldots \ldots \ldots \ldots \ldots \ldots \ldots \ldots$

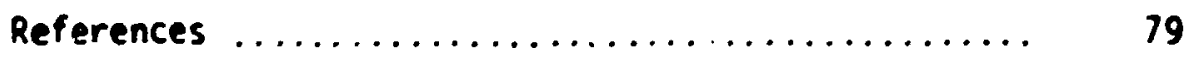




\section{ABSTRACT}

Research activities at The University of Tennessee on gas cooled reactor dynamics are described. The man activity is on steam generator modeling, using approaches ranging from relatively simple linear representation to a detailed nonlinear representation. Model comparisons will involve simulations of the fort St. Vrain reactor stean generator, with emphasis on the evaluation of accuracy vs computation costs. A suller effort is also in progress for modeling the reactor core, the main turbine and the blamer turbines. Preparations are described for using test data from Fort St. Vrain for validating the dynamic models and identifying important design parameters in the plant. 


\section{INTRODUCTION}

The research activities at The University of Tennessee on gas couled eactor dynamics during 1975 are discussed in this progress report.

The main purposes of this work are:

1. component modeling. The emphas is is $r_{i 1}$ steam generator modeling. Several different modeling approaches - anging from a relatively simple few node, linear representation to a detailed multinode, nonlinear representation are being developed and compared. The comparison will involve simularion of the fort St. Vrain steam: generator with each different model and an evaluation of accuracy (relative to the most detailed model) vs. computation costs will be determined. A smaller fffort involving modelinn of other piant components is also in progress. This includes the reactor core, the main turbine and the blower turbines.

2. model validation. Preparations are being made for using test data from fort St. Vrain (to be obtained in a separate cooperative program between reneral Atomic Company and The University of Tennessee) for checking the dynamic models and identifying important design parameters in the plant. This involves methods development for performing the identification and preparation of system rodels for use in the mode?-reference identification. 
CHAPTER I

FORT ST. VRAIN STEAM GEHERATOR MODELING

S. I. Chang

\section{I.I A Doscription of the Systeri}

The fort St. Vrain plant has two identical once-through type steam generator loops arranged in parallel, each of which consists of six individual steam generator modules, located within the cavity cf the prestressed concrete reactor vessel (PCRV) and beneath the reactor core (see Figure 1.1). Each stean-generator module contains a heat-transfer section with a main steam production region and an integral reheater, as $:-11$ as a PCRV penetration section with suitable inlet and outlet connections and helium-pressure barriers.

The steam generator is used to transfer the heat frcm the heliur coolant to the water/steam. The helium flow that cools the reactor core enters the steam generator at high temperature and gives up it.s heat, first to the reheat stean section, then to the once-through main steam/ water section. The sections produce main steam and reheat steam as demanded by the plant load and main turbine requirements.

The main design parameters of the system are shown in Table 1.1. The moitules (Figure 1.2) are divided into three bundles (heating surfaci! sectio:is) arranged one above the other, made up of helically would tuoes supported by perforated plates attached to the central support structure. 


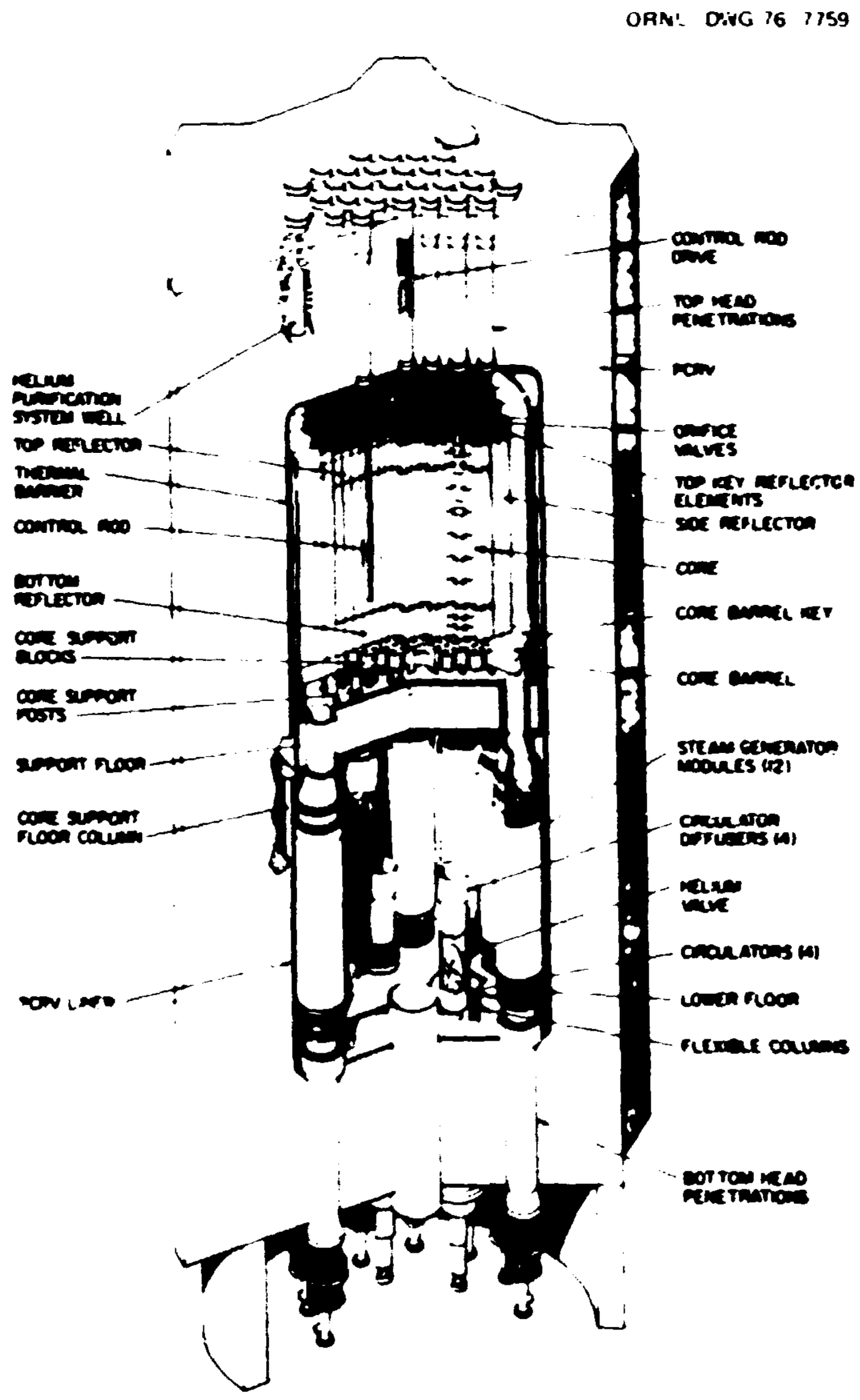

rig. I.1. rort St, Vrain 330-sW(e) HTGR. 
TABLE I. 1

FDRT ST. VPAIN NUCLEAR POWER PLANT

STEAY GENERATOR OESIGN COHDITIONS

100\% Stean Flow

25: Steain Flow

Load

Generator output (M)

342.0

81.2

Station Output (ra)

330.2

67.4

Heliua

Flow (lb/hr)

Outlet Pressure (psia)

Inlet Temperature (F)

Outlet Temperature (F)

Pressure Drop (psi)

$3,452,400$
686
1263
632
3.47

$2,305,326$

2512

403

1005

590

$2,245,366$

600

6?3

1002

42
574,776

2419

299

1000

45

556,928

151

570

1000

Outlet Temperature (F)

Pressure Drop (psi) 


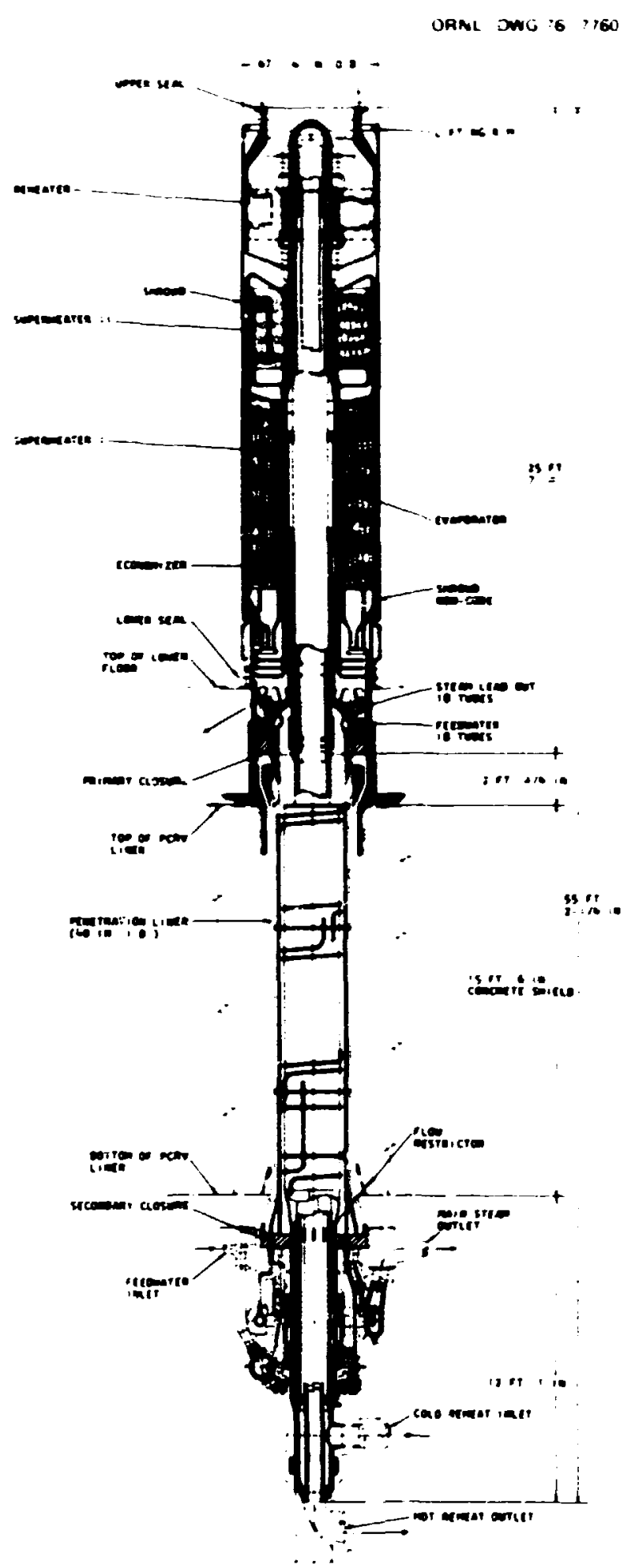

1ig. 1.2. ․t St. Vrain Nuzlear Power Plant Steam Generator Module. 


\section{I.I.I Heliun Circuitry}

Helium leaves the core and traveis dowridard to the steam generator.

The gas then passes over the reheater, finishing superheater, pro-superhecter, evaporator, and economizer surfaces consecutively. Cold helium exits at the botton of the steam generator modules and fiows into the four suction chambers of the helium circu!?t:sre.

\subsubsection{Min Steam Circuitry}

Feedwater is distributed to the 12 modules through flow-control valves and enters each module through a ringheader. The heat-transfer surfaces of the econonizer-evaporator-presuperheater (EES) and finishing superheater (SHil) bundles are composed of helically wound tubes originating at the feedwater subheaders. In the boti:.n EES bundle, the water is preheated, evaporated, and presuperheated. The stean flow is counter current to the helium flow passing over these tubes. Then the crossover tubes lead the presuperheated stean to the finishing superheater (SHII) which is arranged for co-current flow above the EES bundle. This arrangement is required to minimize tube metal temperatures and enhances stability in this section. Superheated steam leaves the SHII bundle via tubes and is collected in the main steam ringheader, which is connected to the main stean piping.

\subsubsection{Integral Reheater $\because$ - cuitry}

cold reheat steam enters each steam-generator nodule through an annulus. The steam is discharged from the annulus to the reheater (RH) bundle. The RH bundic, also composed of helically wound tubes, is 
the uppermost heating surface in the steam generstor, and is arranged for couriter current flow. Hot reheat steam is carried via the central reheat pipe through the primary and secondary closures anj returns to the internedicte pressure $(i D)$ turbine.

\section{I.2 Lumped Parameter A-alysis}

The dynamic analysis of a suosystem sisch as a once through steam generator (OTSG) is a distributed parameter problem. However, a lumped parameter approach is commonly used for its simplicity and ease of solution by either analog or digital techniques. The lumped parameter modeling technique generally results in the physical system being represented in the foliowing form:

$$
\begin{aligned}
& G(x, \dot{x}, t)=F_{1}(x, t)+F_{2}(u, t) \\
& Y(t)=F_{3}(x, u, t) .
\end{aligned}
$$

For dynamic analysis, it is often desiratse to have a linear set of coupled differential equations. Equation (1.2.?) can be linearized by expanding each term in a Taylor Series about in operating point. If the terms above first order are neglected, the result is valid as long as the variables stay ciose to the operating point.

The form of the linearized set of equations is:

$$
\begin{aligned}
A \delta \dot{x} & =B \delta x+C \delta u \\
\delta y & =D \delta x+E \delta u
\end{aligned}
$$

A set of equations of this form was ottained by the System Analysis 
Branch of the Reactor Engineering Department of Genera) Atomic in the mathematical model LAP (Linear Analysis Program).

\section{I.3 Model Fonmulation}

The linear model of LAP includes the primary coolant system, secondary coolant system. and reactor system. Each of these subsysten models was derived from first principles (conservation of mass momentum, encigy, and neutrons) except for an appr;:ination used for the main steam section in the OTSG in early studies. An empirical or transfer function approach was adopted for the main steam section. This empirical model was obtained by tuning arbitrary censtants in LAP's (1) transfer function tyl-model to produce agreement between LAP and TAP. (z)!

The development and implementation of a linear steam generator model derived from first principles was undertaken at GA (work performed by $W$. D. Leech $(3)$ ) because it was believed that an OTSG linear model based on first principles would be superior to the original model for two reasons. First, a model based on first principles yields a better physical unde:-standing. Second, the tuning of the arbitrary constants which appear in the transfer function involved a large degree of qualitative judgement as to when satisfactory agreement was reached hetween TAP and LAP.

In order to account for movement of the beginning and end of the boiling zone, the main steam section was divided into three regions with moving boundaries corresponding to the economizer, evaporator, and superheater. The first node upper boundary was defined as t'ie 
length (from the inlet) required for the water to be heated to saturation. The second node upper boundary, was defined by the additional length required to heat the water from saturated liqiad to saturated vapor. The third node was defined as the remaining length by the steam generator. This formulation allows movement oi the points where boiling begins ( $0:$ quality) and ends (100: quality) and yields a low order representation of the physical systern that retains all of the physical processes usually considered importänt.

\section{I.4. Mathematical Model}

\subsubsection{Assumptions}

In this modeling, the assumptions can be grouped into two sets: those relating to equation developments for each system comporent and those made in obtaini.ng a simplified conceptual system from the real system. More details of system assumptions or afproximations are nade in the development following this section.

Assumptions listed in the follnwing five points refer specificaily to equation development for the $m$-in stean section bundle.

1. Fluid properties a'e uniform at any gi $/ \in n$ cross section.

2. Fluid properties for each node are weighted by the properties at boundaries.

3. No heat conduction occurs in the flow direction.

4. Dynamic effects of gas-pressure changes are negligible, so the gas flow rate is assumed to be time dependent only.

5. Balanced flow and uniform heat flux exist. at any node in the main steam sections. 


\subsubsection{Basic Equations}

Figure I. 3 shows a counter flow heat exchanger. The locations $x_{2}$ and $x_{3}$ are free to move but always remain at fixed quality. For the steam generator model, $x_{2}$ is tre location of saturated liquid (0.3 quality) and $x_{3}$ is the location of saturated vapor (100\% quality).

a. Conservation of Mass.

The basic relationship is:

Flow in - Flow out $=$ rate of change of mass stored.

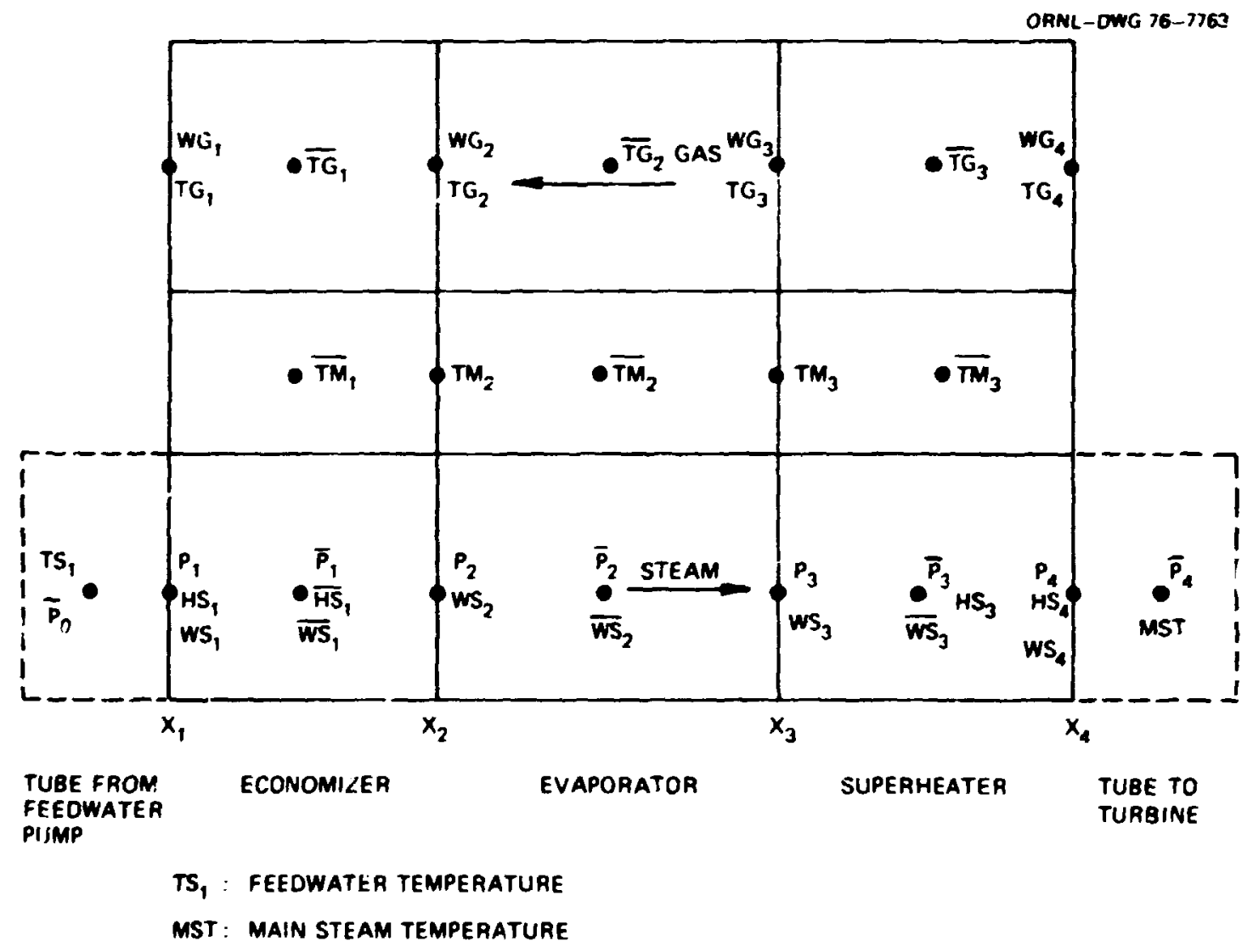

F1g. 1.3. Schematic Representation of a Moving Boundary Model for a Counter Flow Heat Exchanger. 
Gas side

$$
N G_{i}=k G_{\text {in }} \quad(i=1,2,3)
$$

where $H G$ = gas flow rate $(1 \mathrm{~b} / \mathrm{sec})$

$W G_{: n}=$ inlet gas flow rate.

Here we assume the gas flow rate is a function of time only and the same in every section.

\section{Water/steam side}

$$
\begin{gathered}
\left(W S_{i}-\frac{1}{V_{i}} \cdot A \cdot \dot{x}_{i}\right)-\left(W S_{i+1}-\frac{1}{V_{i+1}} \cdot A \cdot \dot{x}_{i+1}\right)= \\
A \cdot \frac{d}{d t}\left[\frac{1}{\bar{v}_{i}}\left(x_{i+1}-x_{i}\right)\right]
\end{gathered}
$$

where

- = irdicated derivative with respect to time

- = indicates average quantity in the node

$W S=$ water $/$ steam flow rate $(1 \mathrm{~b} / \mathrm{sec})$

$V=$ specific volume $\left(\mathrm{ft}^{3} / \mathrm{lb}\right)$

$\vec{v}=$ average specific volume in the node $\left(\mathrm{ft}^{3} / 1 \mathrm{~b}\right)$

$A=$ water/steam flow cross section $\left(\mathrm{ft}^{2}\right)$

$X=$ location along the flow path $(f t)$

$i=1,2,3$. Note that the $x_{1}$ and $x_{4}$ are fixed, so that $\dot{x}_{1}=\dot{x}_{4}=0$ Rewriting Equation (1.4.2)

$$
\begin{aligned}
W S_{i}-W S_{i+1}=A & \cdot \frac{d}{d t}\left[\frac{1}{\bar{v}_{i}} \cdot\left(x_{i+1}-x_{i}\right)\right] \\
& +\frac{1}{v_{i}} \cdot A \cdot \dot{x}_{i}-\frac{1}{v_{i+1}} \cdot A \cdot \dot{x}_{i+1}
\end{aligned}
$$




\section{Conservation of Energy}

The basic relationship $i$.:

Energy flow in - Energy flow out - Rate of energy transferred out $=$ rate of nnergy stored.

a. Gas

$$
\begin{aligned}
& \left(H G_{i+1} \cdot H G_{i+1}+A_{g} \cdot D_{i+1} \cdot H: G_{i+1} \cdot \dot{x}_{i+1}\right)-\left(H G_{i} \cdot H G_{i}+\right. \\
& \left.A_{g} \cdot P_{i} \cdot H G_{i} \cdot \dot{x}_{i}\right)-Q_{g m}=A_{g} \cdot C_{v} \cdot \frac{d}{d t}\left[\bar{o}_{i} \cdot \overline{T G}\left(\bar{x}_{i+1}-x_{i}\right)\right] 1.4 .4
\end{aligned}
$$

where

$$
\begin{aligned}
H G & =\text { enthalpy of gas }(B t u / l b) \\
D_{i} & =\text { gas density }\left(1 b ; \mathrm{ft}^{3}\right) \\
Q_{g m} & =\text { heat transfer rate from gas to metal (Btu/sec) } \\
C_{v} & \left.=\text { constant volume specific heat for the gas (Btu/lb }{ }^{\circ} \mathrm{F}\right) \\
T G & =\text { gas tenperature }\left({ }^{\circ} \mathrm{F}\right)
\end{aligned}
$$

He assume that the helium is an ideal gas under the conditions that exișt in the steam generator. Then

$$
H G=C_{p}(T G+459.6)
$$

where

$$
C_{p}=\text { constant pressure specific heat for the gas }\left(B t u / 1 b^{\circ} \mathrm{F}\right) \text {. }
$$

The gas density can also be related to the gas pressure and gas semperature.

$$
P_{i}=P G_{i} \cdot 0.373343 /\left(T G_{i}+459.6\right)(i=1,2,3,4) . \quad 1.4 .6
$$


Equation (1.4.4) can be rearranged to yield

$$
\begin{aligned}
& W G_{i+1} \cdot H G_{i+1}-H G_{i} \cdot H G_{i}-Q_{g m}=A_{g} \cdot C_{v} \cdot \frac{d}{d t}\left[D_{i} \cdot \overline{T G}_{i} \cdot\right. \\
& \left.\quad\left(x_{i+1}-x_{i}\right)\right] \\
& -A_{g} \cdot p_{i+1} \cdot H G_{i+1} \cdot \dot{x}_{i+1}+A_{g} \cdot o_{i} \cdot H G_{i} \cdot \dot{x}_{i}
\end{aligned}
$$

b. Metal

The basic relationship is:

Heat flow in - Heat flow out = rate of heat storea in the metal.

$$
\begin{gathered}
Q_{g m}-T M_{i} \cdot M \cdot C_{P m} \cdot \dot{x}_{i}-Q_{m s}+T M_{i+1} \cdot M \cdot C_{P m} \cdot \dot{x}_{i+1}= \\
M \cdot C_{P m} \cdot \frac{d}{d t}\left[\overline{T M}_{i} \cdot\left(x_{i+1}-x_{i}\right)\right]
\end{gathered}
$$

where

$$
\begin{aligned}
\overline{T M} & \left.=\text { average metal temperature for the node } i^{\circ} \mathrm{F}\right) \\
C_{P_{m}} & =\text { metai heat capacity }\left(B t u / 1 b-{ }^{\circ} \mathrm{F}\right) \\
Q_{m s} & =\text { heat transfer rate from metal to steam }(B t u / 1 b) \\
M & =\text { metal mass per unit length }(1 \mathrm{~b} / \mathrm{ft}) .
\end{aligned}
$$

We ass'ime the metal boundary temperature to be given by the arithmetic average of adjacent node temperatures

$$
T M_{i}=\frac{1}{2} \cdot\left(\overline{T M}_{i=1}+\overline{T M}_{i}\right) \text { for } i=2,3
$$

Substitute Equation (1.4.9) into (1.4.8) and rearrange to yield

$$
\begin{gathered}
Q_{g m}-Q_{m s}=M \cdot C_{P m} \cdot\left[\left(x_{i+1}-x_{i}\right) \cdot \dot{T M}_{i}+\frac{1}{2}\left(\overline{T M}_{i}-\overline{T M_{i+1}}\right) \cdot\right. \\
\left.\dot{x}_{i+1}-\frac{1}{2} \cdot\left(\overline{T M}_{i}-\overline{T M}_{i-1}\right) \cdot \dot{x}_{i}\right] .
\end{gathered}
$$


13

The heat transfer rates are evaluated as follows:

$$
\begin{aligned}
Q_{g m}= & {\left[\text { Heat transfer area } \cdot\left(\overline{T G}_{i}-\overline{T M}_{i}\right)\right] /\left[\mathrm{KG}_{i} \cdot\left(\overline{W G}_{i}\right)^{-C_{g i}}+1.4 .11\right.} \\
& \left.R_{i} \cdot 0.5\right] \\
Q_{m s}= & {\left[\text { Heat transfer area } \cdot\left(\overline{T M}_{i}-\overline{T S}_{i}\right)\right] /\left[K_{s i} \cdot\left(\overline{W S}_{i}\right)^{-C_{s i}}+\right.} \\
& \left.R_{i} \cdot 0.5\right]
\end{aligned}
$$

where

$$
\begin{aligned}
& K_{g}=\text { constant for gas to metal heat transfer } \\
& C_{g}=\text { flow exponent for gas to metal heat transfer } \\
& K_{S}=\text { constant for metal to water heat transfer } \\
& C_{S}=\text { flow exponent for metal to water heat transfer } \\
& R=\text { thermal resistivity cf the metal times the tube thickness } \\
& T S=\text { water/steam temperature. }
\end{aligned}
$$

C. Water/Steam

$$
\begin{gathered}
\left(W S_{i} \cdot H S_{i}-\frac{1}{V_{i}} \cdot H S_{i} \cdot A \cdot \dot{x}_{i}\right)-\left(H S_{i+1} \cdot H S_{i+1}-\frac{1}{V_{i+1}} \cdot H S_{i+1}\right. \\
\left.A \cdot \dot{x}_{i+1}\right)+Q_{m S}=A \cdot \frac{d}{d t}\left[\frac{1}{\bar{v}_{i}} \cdot \dot{u}_{i} \cdot\left(x_{i+1}-x_{i}\right)\right]
\end{gathered}
$$

where

$$
\begin{aligned}
H S & =\text { enthalpy of water/steam }(B t u / l b) \\
u & =\text { internal energy of water/steam (Btu/lb). }
\end{aligned}
$$

Rearranging Equation (1.4.13) yields:

$$
\begin{aligned}
& W S_{i} \cdot H S_{i}-W S_{i+1} \cdot H S_{i+1}+Q_{m s}=A \cdot \frac{d}{d t}\left[\frac{1}{\bar{v}_{i}} \cdot \bar{u}_{i} \cdot\left(x_{i+1}-x_{i}\right)\right] \\
& +A \cdot\left[-\frac{1}{v_{i+1}} \cdot H S_{i+1} \cdot x_{i+1}+\frac{1}{V_{i}} \cdot H S_{i} \cdot \dot{x}_{i}\right] \cdot
\end{aligned}
$$




\section{Conservation of Momentum}

Since it is assumed the flow on the gas side of the stean generator is time dependent only, there is no need for a momentum equation in that region.

In the water/steam, the basic principle is:

$\sum$ forces $=$ Rate of change of momentum.

$$
\begin{aligned}
P_{i} & -P_{i+1}-K_{f} \overline{W S}_{i}^{2}-\left(\frac{1}{144 \cdot \bar{v}_{i}}\right) \cdot \operatorname{PITCH} \cdot\left(x_{i+1}-x_{i}\right) \\
& =\left(\frac{1}{144 \cdot g \cdot A}\right) \frac{d}{d t}\left[\overline{W S}_{i} \cdot\left(x_{i+1}-x_{i}\right)\right]
\end{aligned}
$$

where

$$
\begin{aligned}
\mathbf{K}_{f} & =\text { friction drop coefficient }\left(\operatorname{PSIA} /(1 \mathrm{bm} / \mathrm{sec})^{2}\right) \\
g & =\text { gravitational constant }\left(\mathrm{ft} / \mathrm{sec}^{2}\right) \\
\text { PITCH } & =\text { height increase per unit length. }
\end{aligned}
$$

In Equation (1.4.15) the first two terms are the pressure fcrce. The third term is tile friction loss. The fourth term is pressure change due to changes in elevation. Due to the coiled nature of the steam generator the PITCH $=0.05$, so we can cancel the fourth term. The coefficient $\left(\frac{1}{144 \cdot g \cdot A}\right)$ of the differential term in the momentum equation is quite small. Furthermore, the sign of $\dot{x}_{i}$ and $\dot{x}_{j+1}$ are the same all the time. So, it can be cancelled without introducing significant error in the model. After cancelling these terms in Equation (1.4.15), we have the general form of the flow pressure drop relation:

$$
\overline{W S}_{i}=\sqrt{\left(P_{i}-P_{i+1}\right) / K_{i}}
$$


where $k_{j}$ is a haad loss coefficient. These algebraic relationships are

$$
\begin{aligned}
& W S_{1}=\sqrt{\left(\bar{P}_{0}-\bar{P}_{1}\right) / K_{1}} \\
& W S_{2}=\sqrt{\left(\overline{P_{1}}-\bar{P}_{2}\right) / K_{2}} \\
& W S_{3}=\sqrt{\left(\overline{P_{2}}-\bar{P}_{3}\right) / K_{3}} \\
& W S_{4}=\sqrt{\left(\overline{\left.P_{3}-\bar{P}_{4}\right) / K_{4}}\right.}
\end{aligned}
$$

d. The weighting factors

The equations which were derived in the above include both the average quantities and end point quantities for the nedes. Additional relationships will be necessary in order to have as many equations as unknowns.

For each node, a weighting factor is defined and the average value for any quantity, $\bar{Y}$, is calculated as follows:

$$
\bar{Y}_{i}=Y_{i} \cdot F_{i}+Y_{i+1} \cdot\left(1-F_{i}\right) \quad F_{i} \leq 1 .
$$

Note that if $F$ equals $0.5, \bar{Y}$ is the linear average. This averaging method is used to calculate $\overline{T G}, \overline{T H}, \overline{W S}, \bar{P}$, and $\overline{H S}$. The average quantities $\bar{U}, \bar{V}$, and $\overline{T S}$ are then calculated from steam tables using $\bar{P}$ and $\overline{H S}$ as the independent variables. The values of $F_{1}=0.75, F_{2}=0.67$ and $F_{3}=0.30$ were used in all the calculations for Section I of this report.

This concludes the derivation of the Iumped nodes equations for a once-through steam generator. Before further development, the assumptions behind the derivation are summarized as follows:

1. Gas flow rate is a function only of time.

2. The enthalpy at $x_{2}$ and $x_{3}$ are functions of pressure with fixed quality. 
3. No heat conduction in the flow direction.

4. Balanced ilow and uniform heat flux exists at any node.

5. In the economizer, the flow in equals flow out.

6. The average water/steam flow rate used in the equation is assumed to be a linear average of the flow in and out of each node.

7. The water/steam flow rate is proportional to the square root of pressure difference.

\subsubsection{Summary of Equations}

Now for the purpose of review, the following equations are a summary of those that must be solved for each node of a moving boundary heat exchanger.

The twelve differential equations are:

$$
\begin{aligned}
W S_{i}-W S_{i+1} & =A\left\{\frac { d } { d t } \left[\frac{l}{\bar{v}_{i}} \cdot\left(x_{i+1}-x_{i}\right)-\frac{1}{v_{i+1}} \cdot \dot{x}_{i+1}\right.\right. \\
& \left.+\frac{i}{v_{i}} \cdot \dot{x}_{i}\right\}(i=1,2,3)
\end{aligned}
$$

Note that $\dot{x}_{1}=\dot{x}_{4}=0$

$$
\begin{aligned}
& W G_{i+1} \cdot H G_{i+1}-W G_{i} \cdot H G_{i}-Q_{g m}=A_{g} \cdot C_{v} \cdot \frac{d}{d t} \cdot \\
& {\left[\bar{o}_{i} \cdot \overline{T G}_{i} \cdot\left(x_{i+i}-x_{i}\right)\right]-A_{g} \cdot \rho_{i+1} \cdot H G_{i+1} \cdot x_{i+1}} \\
& +A_{g} \cdot \rho_{i} \cdot H G_{i} \cdot \dot{x}_{i}(i=1,2,3)
\end{aligned}
$$




$$
\begin{aligned}
& Q_{g m}-Q_{m s}=M \cdot C_{P m} \cdot\left[\left(x_{i+1}-x_{i}\right) \cdot \frac{\dot{T H}}{i}\right. \\
& \left.+\frac{1}{2}\left(\overline{T M}_{i}-\overline{T H}_{i+1}\right) \cdot \dot{x}_{i+1}-\frac{1}{2} \cdot\left(\overline{T H}_{i}-\overline{T M}_{i-1}\right) \cdot \dot{x}_{i}\right] \\
& (i=1,2,3)
\end{aligned}
$$

$$
\begin{aligned}
& W S_{i} \cdot H S_{i}-W S_{i+1} \cdot H S_{i+1}+Q_{m S}=A \cdot \frac{d}{d t}\left[\frac{1}{v_{i}} \cdot \bar{u}_{i} \cdot\right. \\
& \left.\left(x_{i+1}-x_{i}\right)\right]+A \cdot\left[\frac{1}{v_{i}} \cdot H S_{i} \cdot x_{i}-\frac{1}{V_{i+1}} \cdot H S_{i+1} \cdot \dot{x}_{i+1}\right.
\end{aligned}
$$

$$
(i=1,2,3) \text {. }
$$

where $\mathrm{HS}_{2}$ and $\mathrm{HS}_{3}$ are functions of pressure with fixed quality only.

The six algebraic equations are

$$
\begin{aligned}
& W G_{i}=W G_{i n} \quad(i=1,2,3) \\
& W S_{i}=\sqrt{\left(P_{i-1}-\overline{\left.P_{i}\right) / K_{i}}\right.} \quad(i=2,3,4) .
\end{aligned}
$$

The eighteen unknowns for the model are:

1. Three average gas temperature $\left(\overline{F G}_{1}, \overline{T G}_{2}, \overline{T G}_{3}\right)$ described by differenital Equation (1.4.21).

2. Tiree metal temperature $\left(\overline{T H}_{1}, T_{2}, T_{3}\right)$ described by differential Equation (1.4.22).

3. Thiee water/stean pressures $\left(\bar{P}_{1}, \bar{P}_{2}, \bar{P}_{3}\right)$ described by differential Equation (1.4.20).

4. Two locations $\left(x_{2}, x_{3}\right)$ and one enthalpy $\left(H S_{4}\right)$ described by differential Equation (1.4.23). 
5. Three gas flows $\left(W G_{1}, W G_{2}, W G_{3}\right)$ described by algebraic Equation (1.4.24).

6. Three water/steam flow $\left(W_{2}, W S_{3}, W S_{4}\right)$ described by algebraic Equation (1.4.25).

The assumed knowns, or inputs to the problem are:

1. Inlet water pressure $\left(P_{1}\right)$

2. Inlet water flow and enthalpy $\left(W S_{1}, H S_{1}\right)$.

3. Fixed locations $\left(x_{1}, x_{4}\right)$

4. Inlet gas temperature and flow $\left(T G_{4}, H G_{i n}\right)$.

\section{I.5 Linearization of Equations}

The previously derived equations are linearized in the following sections.

\subsubsection{Equation $(1.4 .20)$ Linearization}

Equation (1.4.20) is repe; ted as follows:

$W S_{i}-w S_{i+1}=A\left(\frac{d}{d t}\left[\frac{l}{v_{i}} \cdot\left(x_{i+1}-x_{i}\right)-\frac{l}{v_{i+1}} \cdot \dot{x}_{i+1}+\frac{l}{v_{i}} \cdot \dot{x}_{i}\right\} \quad 1.5 .1\right.$

We assume $\bar{V}_{i}$ is a function of $\overline{H S}_{i}$ and $\bar{p}_{i}$. The average quantities $\overline{\mathrm{HS}}_{i}$ and $\overline{\mathrm{P}}_{i}$ are obtained $b_{j}$ maighting the end point values for each node as follows:

$$
\begin{aligned}
\overline{H S}_{j} & =H_{i} \cdot H S_{i}+\left(1-H_{j}\right) \cdot H S_{i+1} \\
\bar{P}_{i} & =W_{i} \cdot P_{i}+\left(1-W_{j}\right) \cdot P_{i+1} \cdot
\end{aligned}
$$


19

The linearized form of Equation $(1.4 .29)$ is

$$
\begin{gathered}
\delta W S_{i}-\delta W S_{i+1}=A_{1, i} \delta \dot{x}_{i+1}+A_{2, i} \delta \dot{H} S_{i}+A_{3, i} \delta \dot{H} S_{j+1} \\
+\left(A_{4, i}+A_{5, i}\right) \delta \dot{\bar{P}}_{i}+A_{6, i} \delta \dot{x}_{i}
\end{gathered}
$$

where

$$
A_{1, i}=A \cdot\left(\frac{1}{\nabla_{i}}-\frac{1}{V_{i+1}}\right)
$$

(Note: All the values for each variable in the coff icients represent the steady state in the system.)

$$
\begin{array}{rlrl}
L_{i} & =x_{i+1}-x_{i} \\
A_{2, i} & =-A \cdot L_{i} \cdot\left(\frac{\partial \bar{v}_{i}}{\partial H S_{i}}\right)_{P_{i}} \hat{v}_{i}^{2} & i=1 \\
& =0 & i=2,3
\end{array}
$$

$(\bar{V}$ is only a function of pressure when $i=2,3$ ).

$$
\begin{array}{rlr}
A_{3, i} & =-A \cdot L_{i} \cdot\left(\frac{\partial \bar{V}_{i}}{\partial H S_{i+1}}\right)_{P_{i+1}} / \hat{V}_{i}^{2} & i=3 \\
& =0 & i=1,2 \\
A_{4, i} & =-A \cdot L_{i} \cdot\left(\frac{\partial \bar{V}_{i}}{\partial P_{i}}\right)_{H S_{i}} / \hat{v}_{i}^{2} & \\
A_{5, i} & =-A \cdot L_{i} \cdot\left(\frac{\partial \bar{V}_{i}}{\partial P_{i+1}}\right)_{H S_{i+1}} / \bar{v}_{i}^{2} & \\
A_{6, i} & =-A \cdot\left(\frac{i}{V_{i}}-\frac{1}{V_{i}}\right)
\end{array}
$$

$\delta H S_{i}$ can be related to the temperature $\mathrm{TS}_{i}$ as follows:

$$
\delta H S_{i}=\left(\frac{\partial H S_{i}}{\partial P_{i}}\right)_{T S_{i}} \delta P_{i}+\left(\frac{\partial H S_{i}}{\partial T S_{i}}\right)_{P_{i}} \delta T S_{i} \cdot
$$


Since enthalpy is almost independent of pressure for subcooled water, i.e.,

$$
\left(\frac{\partial H S_{1}}{\delta P_{1}}\right)_{T S_{1}}=0 .
$$

For $i=1$, we have

$$
\delta H S_{1}=\left(\frac{\partial i S_{1}}{\partial T S_{1} P_{1}}\right)_{1} \delta S_{1}
$$

The final form of the linearized Equation (I.4.20) is:

ro. $i=1$.

$$
\begin{aligned}
\partial W S_{1} & -\delta W S_{2}=A_{1,1} \delta \dot{x}_{2}+A_{2}, 1 \cdot\left(\frac{\partial H S_{1}}{\partial T S_{1}}\right)_{1}-\delta \dot{T} S_{1} \\
& +\left(A_{4,1}+A_{5,1}\right) \delta \dot{P}_{1}
\end{aligned}
$$

$i=2$

$$
\delta W S_{2}-\delta W S_{3}=A_{6,2} \delta \dot{x}_{2}+\left(A_{4,2}+A_{5,2} j \delta \dot{p}_{2}+A_{1,2} \delta \dot{x}_{3} \cdot \quad 1.5 .5\right.
$$

$\mathbf{i}=3$.

The term $\mathrm{HS}_{4}$ can be related to the main steam temperature $\left(\mathrm{TS}_{4}\right)$ and pressure $\left(\overrightarrow{\mathrm{P}}_{3}\right)$ as follows:

$$
\begin{aligned}
\delta H S_{4} & =\left(\frac{\partial H S_{4}}{\delta \bar{P}_{3}}\right)_{S_{4}} \delta P_{3}+\left(\frac{\partial H S_{4}}{\partial T S_{4}}\right)_{3} \delta S_{4} \\
& =\left(?-W_{3} !\left(\frac{\partial H S_{4}}{\partial P_{4}}\right)_{T S_{4}} \delta \bar{P}_{3}+\left(\frac{\partial H S_{4}}{\partial T S_{4} P_{4}} \delta T S_{4} .\right.\right.
\end{aligned}
$$


After $\delta \mathrm{HS}_{4}$ is replaced by $\& \bar{P}_{3}$ and $\delta T S_{4}$, we have

$$
\begin{aligned}
\delta W S_{3}-\delta W S_{4} & =A_{3,3}\left(\frac{\partial H S_{4}}{\partial T S_{4}}\right)_{4} \delta \dot{T S} S_{4}+G_{1} \delta \dot{\vec{P}}_{3} \\
& +A_{6,3} \delta \dot{x}_{3}
\end{aligned}
$$

where

$$
G_{1}=\left(A_{4,3}+A_{5,3}\right)+A_{3,3} \cdot\left(1-H_{3}\right) \cdot\left(\frac{\partial \mathrm{HS}_{4}}{\partial \mathrm{P}_{4}}\right)_{T_{4}} \cdot
$$

\subsubsection{Equation (1.4.21) Linearization}

tquation (I.4.21) is repeated as follows:

$$
\begin{aligned}
W G_{i+1} & =H G_{i+1}-W G_{i} \cdot H G_{i}-Q_{g m}=A_{g} \cdot C_{v} \cdot \frac{d}{d t}\left[\bar{\rho}_{i} \cdot \overline{T G}_{i} \cdot\left(X_{j+1}-X_{j}\right)\right] \\
& -A_{g} \cdot \rho_{i+1} \cdot H G_{i+1} \cdot \dot{X}_{j+1}+A_{g} \cdot \rho_{i} \cdot H G_{i} \cdot \dot{X}_{i}(i=1,2,3) .
\end{aligned}
$$

We assume $H G$ is a function of TG only and $A$ is constant during a transient. Let

$$
\begin{aligned}
& H G_{i}=C_{p g} \cdot\left(T G_{i}+459.6\right) \\
& \overline{T G}_{i}=T G_{j} \cdot F_{i}+T G_{i+1} \cdot\left(1-F_{i}\right) \\
& \overline{W G}=W G_{i} \cdot F_{i}+W G_{i+1} \cdot\left(1-F_{i}\right) .
\end{aligned}
$$


22

The linearized form of Equation (I.4.21) is as follows

$$
\begin{aligned}
& B_{1, i} \delta l i G_{i+1}+B_{2, i} \delta W G_{i}+B_{3, i} \delta T G_{i+1}+C_{4, i} \delta \overline{T G}_{i} \\
& +B_{4, i} \delta T G_{i}+B_{5, i} \delta x_{i+1}+B_{6, i} \delta x_{i}+B_{7, i} \delta \overline{T M}_{i}= \\
& A_{7, i} \delta \dot{X}_{i+1}+A_{8, i} \delta \dot{X}_{i}+A_{9, i} \delta \dot{\overline{T G}}_{i}
\end{aligned}
$$

where

$$
\begin{aligned}
& E_{1, i}=\pi \cdot 00 \cdot L_{i} \cdot\left(\overline{T G}_{i}-\overline{T M}_{i}\right) \cdot K_{g i} \cdot C_{g i} \cdot W G^{-\left(C_{g i}+1\right)} \\
& \left(K_{g i} \cdot W G^{-C} g i+0.5 R_{i}\right)^{2} \\
& E_{2, i}=\pi \cdot 00 \cdot\left(K_{g i} \cdot W G^{-C_{f i}}+0.5 R_{i}\right)^{-1} \\
& B_{1, i}=H G_{i+1}-E_{1, i} \cdot\left(1-F_{i}\right) \\
& B_{2, i}=-4 G_{i}-E_{1, i} \cdot F_{i} \\
& B_{3, i}=: W G_{i+1} \cdot C_{p g} \\
& C_{4, i}=-E_{2, i} \cdot L_{i} \\
& B_{4, i}=-W G_{i} \cdot C_{P G} \\
& B_{5, i}=-E_{2, i} \cdot\left(\bar{T}_{i}-\overline{T M}_{i}\right) \\
& B_{6, i}=-B_{5, i} \\
& B_{7, i}=E_{2, i} \cdot L_{i} \\
& \text { (cont.) }
\end{aligned}
$$




$$
\begin{aligned}
& A_{7, i}=A_{g} \cdot\left(C_{v g} \cdot \bar{\rho}_{i} \cdot \overline{T G}-\rho_{i+1} \cdot H G_{i+1}\right) \\
& A_{8, i}=-A_{g} \cdot\left(C_{v g} \cdot \bar{\rho}_{i} \cdot \overline{T G}_{i}-\rho_{i}=H G_{i}\right) \\
& A_{9, i}=A_{g} \cdot C C_{v g} \cdot L_{i} \cdot \bar{\rho}_{j}
\end{aligned}
$$

For $i=1$

$$
\begin{aligned}
& B_{1,1} \delta W G_{2}+B_{2,1} \delta H G_{1}+B_{3,1} \delta T_{2}+C_{4,1} \delta \overline{T G}_{1}+B_{4,1} \delta T G_{1} \\
& +B_{5,1} \delta X_{2}+B_{7,1} \delta \overline{T H}_{1}= \\
& A_{7,1} \delta \dot{X}_{2}+A_{9,1} \dot{\delta \overline{T G}} \\
& 1=2 \\
& B_{1,2} \delta W G_{3}+B_{2,2} \delta W G_{2}+B_{3,2} \delta T G_{3}+C_{4,2} \delta \overline{T G}_{2}+B_{4,2} \delta T G_{2} \\
& +B_{5,2} \delta X_{3}+B_{6,2} \delta X_{2}+B_{T, 2} \delta \overline{T M}_{2}= \\
& A_{7,2} \delta \dot{x}_{3}+A_{8,2} \delta \dot{x}_{2}+A_{9,2} i \dot{\bar{T}}_{2} \\
& i=3 \\
& B_{1,3} \delta W G_{4}+B_{2,3} \delta H G_{3}+B_{3,3} \delta T G_{4}+C_{4,3} \delta \overline{T G}_{3}+B_{4,3} \delta T G_{3} \\
& +B_{6,3} \delta X_{3}+B_{7,3} \delta \overline{T M}_{3}= \\
& A_{8,3} \delta \dot{X}_{3}+A_{9,3} \delta \overline{\mathrm{TG}}_{3}
\end{aligned}
$$


Hote that either the variable ST'́ or the variable STG can be eliminated by using Equation (I.5.8). In order to make changes of the weight factor $\left(F_{i}\right)$ in the system dynamic response study convenient, we keep both variables in the equation.

\section{I.5.3 Equation (1.4.22) Linearization}

Equation (1.4.22) is repeated as follows:

$$
\begin{aligned}
& Q_{g m}-Q_{m s}=M \cdot C_{p m} \cdot\left[\left(x_{i+1}-x_{i}\right) \cdot \dot{\overline{T M}}_{i}+\frac{1}{2} \cdot\left(\overline{T M}_{i}-\overline{T M}_{i+1}\right) \cdot\right. \\
& \left.\dot{x}_{i+1}-\frac{1}{2} \cdot\left(\overline{T M}_{i}-\overline{T M}_{i-1}\right) \cdot \dot{x}_{i}\right] \quad(i=1,2,3) .
\end{aligned}
$$

He assume the term $\overline{T S}_{i}$ in $Q_{m s}$ is a function of $\bar{P}_{i}$ and $\overline{H S}_{i}$.

And, let

$$
\begin{aligned}
& \left.\bar{P}_{i}=W_{i} \cdot P_{i}+! ?-W_{j}\right) \cdot P_{i+1} \\
& \overline{H S}_{i}=w_{i} \cdot H S_{i}+\left(1-W_{j}\right) \cdot H S_{i+1} \cdot
\end{aligned}
$$

The linearized form of Equation (1.4.22) as follows:

$$
\begin{aligned}
& B_{8, i} \delta X_{i+1}-B_{8, i} \delta X_{i}+B_{9, i} \delta \overline{T M}_{i}+B_{10, i} \delta \overline{T G}_{i} \\
& +B_{i 2, i} \delta H G_{i+1}+B_{13, i} \delta H G_{i}+\left(B_{14, i}+B_{15, i}\right)_{i} \delta \bar{P}_{i} \\
& +B_{16, i} \delta H J,+B_{17, i} \delta H S_{i+1}+B_{18, i} \delta N S_{i}+B_{19, i} \delta^{\prime} / S_{i+1} \\
& =A_{11, i} \delta \overline{T M}_{i}+A_{12, i} \delta \dot{X}_{i+1}+A_{13, i} \delta \dot{X}_{i}
\end{aligned}
$$


25

where

$$
\begin{aligned}
& E_{4, i}=.00 \cdot\left(\mathrm{KS}_{i} \cdot \mathrm{WS^{-CS }}+0.5 R_{i}\right)^{-1} \\
& E_{5, i}=0.00 \cdot L_{i} \cdot k S_{i} \cdot C S_{i} \cdot W s^{-\left(C S_{i}+1\right)} \cdot\left(T H_{i}-T S_{i}\right) \\
& \cdot\left(K S_{i} \cdot i S^{-C S}+0.5 R_{i}\right)^{-2} \\
& s_{8,1}=E_{2,1} \cdot\left(T_{C_{1}}-M_{1}\right)-E_{4,1} \cdot\left(T_{i}-T_{1}\right)=0
\end{aligned}
$$

(For at the steady state, the heat transferred in equals the heat transferred out.)

$$
\begin{aligned}
& B_{9, i}=-E_{i, i} \cdot L_{i}-E_{4, i} \cdot L_{i} \\
& B_{10, i}=E_{2, i} \cdot L_{i} \\
& B_{12, i}=E_{1, i} \cdot\left(1-F_{i}\right) \\
& B_{13, i}=E_{1, i} \cdot F_{i} \\
& B_{14, i}=E_{4, i} \cdot\left(\frac{\partial S_{i}}{\partial P_{i}}\right)_{S_{i}} \cdot L_{i} \\
& B_{15, i}=E_{4, i} \cdot\left(\frac{\partial T S_{i}}{\partial P_{i+1}}\right)_{i S_{i+1}} \cdot L_{i} \\
& B_{16, i}=E_{4, i} \cdot\left(\frac{\partial S_{i}}{\partial H S_{i}} P_{i} \cdot L_{i} \quad i=1\right. \\
& =0
\end{aligned}
$$

(For $1=2,3, T S_{i}$ is function of $p_{i}$ only with fixed quality.) 


$$
\begin{array}{rlrl}
B_{17, i} & =E_{4, i} \cdot\left(\frac{\partial \overline{T S}_{i}}{\partial H S_{i+1}}\right)_{P_{i+1}} \cdot L_{i} & i=3 \\
& =0 & i=1,2
\end{array}
$$

$$
\begin{aligned}
& B_{18, i}=-E_{5, i} \cdot H_{i} \\
& B_{19, i}=-E_{5, i} \cdot\left(1-H_{i}\right)
\end{aligned}
$$$$
A_{11, i}=H \cdot C_{p m} \cdot L_{i}
$$$$
A_{12, i}=\frac{1}{2} M \cdot C_{p m} \cdot\left(\overline{T M}_{i}-\overline{T M}_{j+1}\right)
$$$$
A_{13, i}=-\frac{1}{2} M \cdot c_{p m} \cdot\left(\overline{T M}_{i}-\overline{T M}_{i-1}\right) .
$$

The final linearized equations for each node are:

$$
\begin{aligned}
& \text { For } \mathbf{i}=1 \\
& B_{9,1}+\delta \overline{T H}_{1}+B_{1 C, 1} \delta \overline{T G}_{1}+B_{12,1} \delta H G_{2}+B_{13,1} \delta H G_{1} \\
& +\left(B_{14,1}+B_{15,1}\right) \delta \bar{p}_{1}+B_{16,1} \cdot\left(\frac{\partial H S_{1}}{\partial T S_{1}}\right)_{p_{1}} \cdot \delta T S_{1}+B_{18,1} \delta W S_{1} \\
& +B_{19,1} \delta W S_{2}=A_{11,1} \delta \dot{\overline{T M}}+A_{12, i} 5 x_{2}
\end{aligned}
$$


27

$$
\begin{aligned}
& i=2 \\
& B_{9,2} \delta T \bar{M}_{2}+B_{10,2} \delta T G_{2}+B_{12,2} \delta H G_{3}+B_{13,2} \delta H G_{2} \\
& +\left(B_{14,2}+B_{15,2}\right) \delta \bar{P}_{2}+B_{18,2} \delta W S_{2}+B_{19,2} \delta W S_{3} \\
& =A_{11,2} \delta T \bar{H}_{2}+A_{12,2} \delta \dot{x}_{3}+A_{13,2} \dot{\delta} \dot{x}_{2}
\end{aligned}
$$

$$
\begin{aligned}
i=3 & B_{9,3} \delta \overline{T H}_{3}+B_{10,3} \delta \overline{T G}_{3}+B_{12,3} \delta N G_{4}+B_{13,3} \delta H G_{3} \\
& +G_{2} \delta \bar{P}_{3}+B_{17,3} \cdot\left(\frac{\partial H S_{4}}{\partial T S_{4}}\right)_{4} \cdot \delta T_{4}+B_{18,3} \delta H S_{3}+B_{19,3} \delta \cdot N S_{4} \\
& =A_{11,3} \delta \dot{\overline{T H}}_{3}+A_{13,3} \delta \dot{x}_{3}
\end{aligned}
$$

where

$$
G_{2}=\left[B_{14,3} \pm B_{15,3}+B_{17,3} \cdot\left(1-W_{3}\right) \cdot\left(\frac{\partial H S_{4}}{\partial P_{4}}\right)_{T S_{4}}\right] .
$$

I.5.4 Equation (1.4.23) Linearization

Equation (1.4.23) is repeated as follows:

$$
\begin{gathered}
W S_{i} \cdot H S_{i}-W S_{i+1} \cdot H S_{i+1}+Q_{m S}=A \cdot \frac{d}{d t}\left[\frac{1}{\tilde{V}_{i}} \cdot \tilde{U}_{i} \cdot\right. \\
\left.\left(x_{i+1}-x_{i}\right)\right]+A \cdot\left[\frac{1}{V_{i}} \cdot H S_{i} \cdot \dot{x}_{i}-\frac{1}{V_{i+1}} \cdot H S_{i+1} \cdot \dot{x}_{i+1}\right] \\
(i=1,2,3) .
\end{gathered}
$$

.1 .5 .15

Assume the $\bar{U}_{i}$ and $\bar{V}_{i}$ are functions of $\bar{P}_{i}$ and $\overline{H S}_{i}$.

Let

$$
\delta H S_{i}=\left(\frac{\partial H S_{j}}{\partial P}\right)_{\text {quality }} \cdot \delta P_{i} \quad(i=2,3) .
$$


28

Note: It is $0:$ quality at boundary 2 and $100 \%$ quality at boundary 3. The linearized form of Equation (1.4.23) is as follows:

$$
\begin{aligned}
& B_{20, i} \delta H S_{i}+B_{21, i} \delta 4 S_{i}+B_{22, i} \delta H S_{i+1}+B_{23, i} \delta i S_{i+1} \\
& -B_{24, i} \delta X_{i+1}+B_{24, i} \delta X_{i}-B_{25, i} \delta \overline{M M}_{i}+B_{26, i} \delta P_{i} \\
& +B_{27, i} s{ }_{i+1}+B_{23, i} \delta \bar{P}_{i-1}=A_{14, i}+s H S_{i}+A_{16, i} i \dot{H} S_{i+1} \\
& +\left(A_{15, i}+A_{17, i}\right) \dot{s} \dot{\bar{P}}_{i}+A_{18, i} \delta \dot{X}_{i+1}+A_{19, i} \delta \dot{X}_{i}
\end{aligned}
$$

where

$$
\begin{array}{rlrl}
B_{20, i} & =H S_{i}-E_{4, i} \cdot\left(\frac{\partial \overline{T S}_{i}}{\partial H S_{i}}\right) \cdot L_{i} & i=1 \\
& =0 & i=2,3 \\
B_{21, i} & \left.=H S_{i}+H_{i} \cdot E_{5, i} \frac{\partial \overline{T S}_{i}}{\partial H S_{i+1}}\right) \cdot L_{i} \quad i=3 \\
B_{22, i} & =-H S_{i+1}-E_{4, j}\left(\frac{i=1,2}{}\right. & =0 &
\end{array}
$$

$$
\begin{gathered}
B_{23, i}=-H S_{i+1}+\left(1-W_{i}\right) \cdot E_{5, i} \\
B_{24, i}=-E_{4, i} \cdot\left(\overline{T M}_{i}-\overline{T S}_{i}\right) \\
B_{25, i}=-E_{4, i} \cdot L_{i} \\
D_{i}=-E_{4, i} \cdot L_{i} \cdot\left[\left(\frac{\left.\partial \overline{T S}_{i}\right)_{i} H S_{i}}{\partial \overline{P P}_{i+1} H S_{i+1}}\right]\right. \\
\text { In } B_{26, i}, B_{27, i} \text {, and } B_{28, i} \text {, we let } \\
\delta P_{i}=\frac{1}{2}\left(\delta \bar{P}_{i-i}+\delta \bar{P}_{i}\right)
\end{gathered}
$$


29

$$
\begin{aligned}
& \text { for } i=1 \\
& B_{26,1}=D_{1}-\frac{1}{2} \cdot W S \cdot\left(\frac{\partial H S_{2}}{\partial P_{2}}\right)_{\text {quality }} \\
& B_{27,1}=-\frac{1}{2} \cdot W S \cdot\left(\frac{\partial H S_{2}}{\partial P_{2}}\right)_{\text {quality }} \\
& B_{28,1}=0 \\
& i=1 \\
& B_{26,2}=D_{2}-\frac{1}{2} \cdot W S \cdot\left[\left(\frac{\partial H S_{3}}{\partial P_{3}}\right)_{\text {quality }}-\left(\frac{\partial H S_{2}}{\partial P_{2}}\right)_{\text {equal ito }}\right] \\
& B_{27,2}=-\frac{1}{2} \cdot H S \cdot\left(\frac{\partial H_{3}}{\partial P_{3}}\right)_{\text {quality }} \\
& B_{28,2}=\frac{1}{2} \cdot W S \cdot\left(\frac{\partial H S_{2}}{\partial P_{2}}\right)_{\text {quality }} \\
& i=3 \\
& B_{26,3}=D_{3}+\frac{1}{2} \cdot W S \cdot\left(\frac{\partial H S_{3}}{\partial P_{3}}\right)_{\text {quad itiy }} \\
& B_{27,3}=0 \\
& B_{28,3}=\frac{1}{2} \cdot W S \cdot\left(\frac{\mathrm{OHS}_{3}}{\partial \mathrm{P}_{3}}\right)_{\text {quality }} \\
& A_{14, i}=A \cdot L_{i} \cdot \nabla_{i}^{-2} \cdot\left[\bar{V}_{i} \cdot\left(\frac{\partial \bar{W}_{i}}{\partial H S_{i}}\right)_{P_{i}}-\bar{U}_{i} \cdot\left(\frac{\partial \bar{V}_{i}}{\partial H S_{i}}\right)_{P_{i}}\right] i=1 \\
& =0 \\
& A_{15, i}=A \cdot L_{i} \cdot V_{i}^{-2} \cdot\left[\bar{v}_{i}\left(\frac{\partial \bar{U}_{i}}{\partial P_{i}}\right)_{S_{i}}-O_{i}\left(\frac{\partial \nabla_{i}}{\partial P_{i}}\right)_{H S_{i}}\right] \\
& A 17, i=A \cdot L_{i} \cdot \nabla_{i}^{2} \cdot\left[\nabla_{i}\left(\frac{\partial \bar{D}_{i}}{\partial P_{i+1}}\right)_{H S_{i+1}}-O_{i}\left(\frac{\partial \bar{V}_{i}}{\partial P_{i+1}}\right)_{H S_{j+1}}\right]
\end{aligned}
$$


30

$$
\begin{aligned}
A_{16, i} & =A \cdot L_{i} \cdot \nabla_{i}^{2} \cdot\left[\nabla_{i} \cdot\left(\frac{\partial \bar{U}_{i}}{\partial H S_{i+1}}\right)_{P_{i+1}}-O_{i} \cdot\left(\frac{\partial \bar{V}_{i}}{\partial H S_{i+1}}\right)_{P_{i+1}}\right] i=3 \\
& =0 \quad i=1,2 \\
A_{18, i} & =A\left[\frac{\bar{U}_{i}}{\bar{V}_{i}}-\frac{H S_{i+1}}{V_{i+1}}\right] \\
A_{19, i} & =A\left[\left[\frac{\bar{U}_{i}}{\bar{v}_{i}}-\frac{H S_{i}}{V_{i}}\right] .\right.
\end{aligned}
$$

The linearized equations for each node are:

$$
\text { for } \begin{aligned}
i & =1 \\
& B_{20,1} \cdot\left(\frac{\partial H S_{1}}{\partial T S_{1}}\right)_{P_{1}} \cdot S T S_{1}+B_{21,1} \delta H S_{1}+B_{23,1} \delta H S_{2} \\
& -B_{24,1} \delta X_{2}-B_{25,1} \delta T \bar{H}_{1}+B_{26,1} \delta P_{1}+B_{27,1} \delta P_{2} \\
& =A_{14,1} \cdot\left(\frac{\partial H S_{1}}{\partial T S_{1}}\right)_{P_{1}} j T S_{1}+\left(A_{15,1}+A_{17,1}\right)_{S \dot{P}_{1}}+A_{12,1} \delta \dot{X}_{2}
\end{aligned}
$$

$$
\mathbf{i}=\mathbf{2}
$$

$$
\begin{aligned}
& B_{21,2} \delta H S_{2}+B_{23,2} \delta H S_{3}-B_{24,2} \delta X_{3}+B_{24,2} \delta X_{2} \\
& -B_{25,2} \delta T \overline{T H}_{2}+B_{26,2} \delta P_{2}+B_{27,2} \delta \dot{F}_{3}+B_{28,2} \delta P_{1} \\
& =\left(A_{15,2}+A_{17,2}\right) \delta \dot{P}_{2}+A_{18,2} \delta \dot{x}_{3}+A_{19,2} \delta \dot{x}_{2}
\end{aligned}
$$

1.5 .16

$i=3$

$$
\begin{aligned}
& B_{21,3} \delta H S_{3}+B_{22,3} \cdot\left(\frac{\partial H S_{4}}{\partial T S_{4}}\right)_{P_{4}} \delta T S_{4}+B_{23,3} \delta H S_{4}+B_{24,3} \delta X_{3} \\
& -B_{25,3} \delta T H_{2}+G_{3} \delta P_{3}+B_{28,3} \delta P_{2} \\
& =G_{4} \delta \dot{P}_{3}+A_{16,3} \cdot\left(\frac{\partial H S_{4}}{\partial T S_{4}}\right)_{P_{4}} \cdot \delta T S_{4}+A_{19,3} \delta \dot{X}_{3}
\end{aligned}
$$


where

$$
\begin{aligned}
& G_{3}=B_{26,3}+\left(1-H_{3}\right) \cdot B_{22,3} \cdot\left(\frac{\partial H S_{4}}{\partial P_{4}}\right)_{T_{4}} \\
& G_{4}=A_{15,3}+A_{17,3}+\left(1-H_{3}\right) \cdot A_{16,3} \cdot\left(\frac{\partial H S_{4}}{\partial P_{4}}\right)_{T_{4}} \cdot
\end{aligned}
$$

The nine linearized algebraic equations are

$$
\begin{array}{lll}
\delta H G_{i}=\delta H G_{i n} \quad(i=1,2,3) & I .5 .18 \\
\delta \overline{T G}_{i}=F_{i} \cdot \delta T G_{i}+\left(1-F_{i}\right) \cdot \delta T G_{i+1} \quad(i=1,2,3) & I .5 .19 \\
\delta H S_{2}=K K_{2} \cdot\left(\delta \bar{P}_{1}-\delta F_{2}\right) & I .5 .20
\end{array}
$$

where

$$
\begin{aligned}
& K K_{2}=\frac{H S}{2\left(\bar{P}_{1}-\bar{P}_{2}\right)} \\
& \delta W S_{3}=K K_{3} \cdot\left(\delta \tilde{P}_{2}-\delta \tilde{P}_{3}\right)
\end{aligned}
$$

where

$$
\begin{aligned}
& K K_{3}=\frac{W S}{2\left(P_{2}-P_{3} j\right.} \\
& \delta H S_{4}=K K_{4} \cdot\left(\delta \bar{P}_{3}-\delta \bar{P}_{4}\right)
\end{aligned}
$$

where

$$
K K_{4}=\frac{W S}{2\left(P_{3}-P_{4}\right)} .
$$

The linearized equations from (1.5.4) to (I.5.22) can then be arranged in a matrix form. 


\section{I.6 Results and Discussion}

The linearized equations presented in the above section were used to calculate the frequency response and time response of the main steam generator (no reheater) for selected inputs. In obtaining these results, two different sets of equations were used. In the first set, the feedwater pressure, $P_{0}$, (see figure I.3) is held constant; hence the description "ronstant pressure model" (CPSG). In the second set, the variable WS, (fesdwater flow) is held constant; hence, the description "constint. flow mouel" (CFSG). The frequency response and time responses for the constant pressure and constant flow models were calculated for e. in input selected.

The code SFRino (5) was used to calculate the frequency res onse. The input to SFRMOD is of the form:

$$
A_{1} \frac{d \bar{x}}{d t}+A_{2} \bar{x}=\bar{f}
$$

where

$$
\begin{aligned}
& \begin{aligned}
& A_{1}= \text { matrix of constant coefficients related to differential } \\
& \text { te 'ms } \\
& A_{2}= \text { matrix of constant coefficients related to algebraic } \\
& \text { terms } \\
& \bar{x}= \text { vectors of systen variables } \\
& 7= \text { forcing vector for the selectrd input. } \\
& \text { Before calculating the time response, } i t \text { was necessary to }
\end{aligned} \\
& \text { transfer the system of algebraic and differential equations (I.6.1) }
\end{aligned}
$$


describing the steam generator to the following form

$$
\frac{d \bar{X}}{d t}=A \bar{X}_{d}+F_{d}
$$

where

$$
\begin{aligned}
\bar{x}_{d} & =\text { vector of state variables } \\
A & =\text { matrix of constant coefficients } \\
\overline{\mathbf{f}}_{d} & =\text { forcing terms for the system input. }
\end{aligned}
$$

This was performed using the computer code PUREDIFF. (5) The input to PUREDIFF consists of the $A_{1}$ and $A_{2}$ matrices and $\bar{f}$ vector as in Equation (I.6.1). The $A_{1}, A_{2}$, and $A$ ma irices appear in Table I.2

\begin{tabular}{|c|c|}
\hline Number & Veriables \\
\hline $1,2,3$ & $\delta \bar{P}_{1}, \delta \bar{P}_{2}, \delta \bar{P}_{3}$ \\
\hline $4,5,6,7$ & $\delta T G_{1}, \delta T G_{2}, \delta T G_{3}, \delta T G_{4}$ \\
\hline $8,9,10$ & $\delta \overline{T M}_{1}, \delta \overline{T M}_{2}, \delta \overline{T M}_{3}$ \\
\hline 11,12 & $\delta x_{2}, \delta x_{3}$ \\
\hline 13 & SMST \\
\hline $14,15,16$ & $\delta \overline{T G}_{i}, \delta \overline{T G}_{2}, \delta \overline{T G}_{3}$ \\
\hline $17,18,19,20$ & $\delta W S_{1}, \delta W S_{2}, \delta W S_{3}, \delta W S_{4}$ \\
\hline
\end{tabular}
and Table I.3.

TABLE I.2

THE VARIABLES ASSOCIATED WITH CPSG FOR $A_{1}$ AND $A_{2}$ MATRIX 
TABLE I.3

THE STATE VARIABLES ASSOCIATED HITH CPSG FOR REDUCED A MTRIX

\begin{tabular}{ll}
\hline Nimber & State Variables \\
\hline $1,2,3,4$ & $\delta \mathrm{P}_{1}, \delta \bar{P}_{2}, \delta \mathrm{P}_{3}, \delta \mathrm{TG}_{4}$ \\
$5,6,7$ & $\delta \overline{\mathrm{TH}}_{1}, \delta \overline{\mathrm{TM}}_{2}, \delta \overline{T T H}_{3}$ \\
$8, \mathrm{~s}$ & $\delta \mathrm{X}_{2}, \delta \mathrm{X}_{3}$ \\
10 & $\delta \mathrm{HST}$ \\
$11,12,13$ & $\delta \overline{T G}_{1}, \delta \mathrm{TG}_{2}, \delta \overline{T G}_{3}$
\end{tabular}

\begin{tabular}{|c|c|c|c|c|c|}
\hline \multicolumn{6}{|c|}{$A I(I, J)$} \\
\hline 1 & $12.61053 E-01$ & 1 & $113.38096 \mathrm{E} 01$ & 2 & $21.01479 \mathrm{E} \mathrm{OV}$ \\
\hline 2 & $112.28625 E \quad 01$ & 2 & $121.47444 \mathrm{E} 03$ & 3 & $33.13884 E-01$ \\
\hline 3 & $12 \quad 1.05238 E \quad 01$ & 3 & $13-5.05814 E-01$ & 7 & 71.00000 E 00 \\
\hline 8 & $87.41049 E 03$ & y & $11-7.91655 \mathrm{E} 03$ & 9 & $94.71117 E 03$ \\
\hline 9 & $11-7.91655 E_{03}$ & 9 & $12-1.09647 \mathrm{E} 04$ & 10 & $107.51123 E 03$ \\
\hline 10 & $12 \cdot 1.09647 \mathrm{E} \quad 04$ & 11 & I $4.14207 \mathrm{E} 02$ & 11 & $11-6.98903 E 02$ \\
\hline 12 & $29.45497 E^{2} .2$ & 12 & $111.57921 E 04$ & 12 & $128.68772 \mathrm{E} 03$ \\
\hline 13 & $33.67355 E 02$ & 13 & $129.52171 \mathrm{E} 03$ & 13 & $13-4.25746 E 02$ \\
\hline 14 & $11-3.11099 E 02$ & 14 & $143.67501 E$ o1 & 15 & 113.11099 E 02 \\
\hline 15 & 12-3.11099E 02 & 15 & $15 \quad 1.97891 \mathrm{E} 01$ & i6 & $123.11099 E 02$ \\
\hline 16 & $16 \quad 2.59237 E 01$ & & & & \\
\hline
\end{tabular}

\begin{tabular}{|c|c|c|c|c|c|}
\hline \multicolumn{6}{|c|}{$A 2(I, J)$} \\
\hline 1 & $17-1.00000$ E 00 & I & $181.00000 E 00$ & 2 & $18-1.00000 E 00$ \\
\hline 2 & 19 1. COGOOE 00 & 3 & $19-1.00000 \mathrm{E} 00$ & 3 & 201.00000 E0 \\
\hline 4 & $6-7.50000 E-01$ & 6 & $5-2.50000 E-01$ & 4 & $141.00000 E 00$ \\
\hline 5 & $5-6.70000 E-01$ & 5 & $6-3.30000 E-0 i$ & 5 & $151.00000 E 00$ \\
\hline ó & $6-3.00000 E-\cap 1$ & 6 & $7-7.00000 \mathrm{E}-01$ & 6 & $161.00000 \mathrm{E} 00$ \\
\hline 7 & ;2.00000E 00 & 8 & $1-8.77428 \mathrm{E} 01$ & 8 & $83.54908 \mathrm{E} 03$ \\
\hline 8 & $16-9.37232 E \quad 02$ & 8 & $178.58445 E 01$ & 8 & $182.86148 \mathrm{E} \quad 01$ \\
\hline 9 & $2-9.14281 E 01$ & 9 & $92.35927 E, 03$ & 9 & $15-5.95840 \mathrm{E} 02$ \\
\hline 9 & $184.74777 E 01$ & 9 & $192.33845 \mathrm{E} 01$ & i0 & $31.42560 \mathrm{E} 02$ \\
\hline 10 & $10 \quad 3.89902 \mathrm{E} \quad 03$ & 10 & $13-1.83209 \mathrm{E} \quad 03$ & 10 & 16-9.4997 उE 02 \\
\hline 10 & $193.52938 \mathrm{E} 01$ & 10 & $208 . .3522 E 01$ & 11 & $11.13677 E 02$ \\
\hline 11 & $22.59346 E 01$ & 11 & $8-2.61185 E 03$ & 11 & $11-1.39502 \mathrm{E} 03$ \\
\hline 11 & $17-4.71644 \mathrm{E} 02$ & 11 & $187.48713 E 02$ & 12 & $1-2.59346 \mathrm{E} 01$ \\
\hline 12 & $23.78754 E 01$ & 12 & $3-2.76181 E 01$ & 12 & $9-1.763435,03$ \\
\hline 12 & $11 \quad 1.54129 \mathrm{E} \quad 03$ & 12 & $12-1.54129 \mathrm{E} \quad 3$ & 12 & $18-8.24805 E 02$ \\
\hline 12 & $19 \quad 1.02896 \mathrm{E} \quad 03$ & 13 & $22.76181 \mathrm{E} 01$ & 13 & $3-1.25829 \mathrm{E} \quad 02$ \\
\hline 13 & $10-2.94904 E 03$ & 13 & $121.8: 334 E 03$ & 13 & 132.04697 E 03 \\
\hline 13 & 19-1.08763E 03 & 13 & $201.48584 \mathrm{E} 03$ & 14 & $45.95539 \mathrm{E} 02$ \\
\hline 14 & S-5.95539E 02 & 14 & $8-9.37232 E 02$ & 14 & $111.45888 \mathrm{E} 03$ \\
\hline 14 & $149.37232 E \quad 02$ & 15 & S $5.95539 E 02$ & 15 & $6-5.95539 \mathrm{E} 02$ \\
\hline 15 & $9-5.95840 E 02$ & is & $11-1.61186 i 01$ & 15 & $121.61186 \mathrm{E} 03$ \\
\hline $1 \%$ & $15 \quad 5.95840 E 02$ & 16 & $65.95539 \mathrm{E} 02$ & 15 & $1-5.95539 E 02$ \\
\hline 16 & 10-\$.49973E 02 & 16 & $12-1.89635 E 03$ & 16 & $160.49973 E \quad 02$ \\
\hline 17 & $16.18079 E 00$ & 17 & $171.00000 \mathrm{E} 00$ & 10 & $1-7.24500 E D 0$ \\
\hline 18 & $27.24500 F .00$ & 18 & $181.00000 E 00$ & 19 & $\ddot{2}-2.85951 \mathrm{E} 00$ \\
\hline 19 & $3: .85951 F 00$ & 19 & $191.00000 \mathrm{E} 00$ & 20 & $3-1.37705 E 00$ \\
\hline 20 & $201.00000 E O O$ & & & & \\
\hline
\end{tabular}


TABLE $\cdot 3$ (Cont inued)

\begin{tabular}{|c|c|c|c|c|c|c|c|}
\hline & REDLCED Al-FATR DX & IS & & & & & \\
\hline 1 & $1-2.0806900 \mathrm{E} 01$ & 1 & $21.3222581 \mathrm{~F} 01$ & 1 & $56.2250166 E 00$ & 1 & $83.3246164 \mathrm{E} 00$ \\
\hline 2 & $16.5625935 E 00$ & 2 & $2-8.12296 ? 5 \mathrm{E} 00$ & 2 & 32.4341621500 & 2 & $53.2062525 \mathrm{E}-01$ \\
\hline 2 & $65.0771427 \mathrm{E} 00$ & 2 & $8-4.2665825 E 00$ & 2 & $94.4378195 \mathrm{E} 00$ & 3 & $1-2.629428$ SE 00 \\
\hline 3 & $25.6758375 E 00$ & 3 & $3-1.1549730 \mathrm{E} 01$ & 3 & $5-3.3963531 E-01$ & 3 & $62.2627077 \mathrm{E} 00$ \\
\hline 3 & $72.8572784 \mathrm{E} \mathrm{Ol}$ & 3 & $8-2.1591740 E 00$ & 3 & $9-1.5588333 \mathrm{E}$ 01 & 3 & $10-1.9833328 \mathrm{E} 01$ \\
\hline 4 & $4-2.0000000 E 00$ & 5 & $1-1.9 ; 12412 \mathrm{E}-01$ & 5 & $21.4782137 \mathrm{E}-01$ & 5 & $5-5.3030968 E-01$ \\
\hline 5 & $8-2.7431257 \mathrm{E}-02$ & 5 & $111.2647772 \mathrm{E}-01$ & 6 & $14.7485876 \mathrm{E}-01$ & 6 & $2-2.6879144 E-01$ \\
\hline 6 & $3-8.7153971 E-02$ & 6 & $54.1298445 E-02$ & 6 & $6-1.3141069 E 00$ & 6 & $87.3300046 \mathrm{E}-01$ \\
\hline 6 & $9-7.1094406 \mathrm{E}-01$ & 6 & $121.2646991 \mathrm{E}-01$ & 7 & $15.9283441 \mathrm{E}-01$ & 7 & $2-3.4774709 E-01$ \\
\hline 7 & J-0.: $: 97608 E-02$ & 7 & $57.6574564 \mathrm{E}-02$ & 7 & G-5.1015300E-01 & 7 & $7-5.1910526 \mathrm{E}-01$ \\
\hline 7 & $84.8681015 E-01$ & $?$ & $9-4.4591367 \mathrm{E}-01$ & $i$ & $102.4392888 \mathrm{E}-01$ & 7 & $131.2646112 \mathrm{E}-01$ \\
\hline 8 & $1-2.364: 872 E-01$ & 8 & $21.1217344 \mathrm{E}-01$ & 8 & $5-4.8073113 E-02$ & 8 & $8-2.5674574 E-02$ \\
\hline 9 & $14.0627545 E-01$ & 3 & $2-2.3232561 E-01$ & 9 & $3-4.1429687 \mathrm{E}-02$ & 9 & $5 \quad 5.2477356 \mathrm{E}-02$ \\
\hline 9 & $6-3.4961307 E-01$ & 9 & $83.3361596 \mathrm{E}-01$ & 9 & $9-3.0558920 \mathrm{E}-01$ & 10 & $16.8181896 \mathrm{E} 00$ \\
\hline 10 & $2-4.9869909 E \mathrm{E}$ & 10 & $3-1.6296976 \mathrm{E} \quad 00$ & 10 & $58.8068461 E-01$ & 10 & $6-5.8672705 E 00$ \\
\hline 10 & $71.773230 \mathrm{GE} 01$ & 10 & $\begin{array}{ll}85.5988035 E \quad 00\end{array}$ & 10 & $9-1.6029999 E$ O1 & 10 & $10-1.2308591 \mathrm{E} 01$ \\
\hline 11 & $1-2.0013561 E 00$ & 11 & $29.4958210 \mathrm{E}-01$ & 11 & $42.4830109 \mathrm{E} 01$ & 11 & $52.5095078 \mathrm{E} 01$ \\
\hline 11 & $8-3.9318015 E 01$ & 11 & $11-4.7107468 \mathrm{E} 01$ & 11 & $123.2246902 \mathrm{E} 01$ & $1:$ & $13-3.5471603 \mathrm{E} 01$ \\
\hline 12 & $11.0103191 E$ ol & 12 & $2-5.4155455 E \quad 00$ & 12 & $3-6.5127712 E-01$ & 12 & $4-1.0479436 E C ?$ \\
\hline 12 & $51.5806589 E 00$ & 12 & $62.4610168=01$ & 12 & $88.7103363 \mathrm{E} 01$ & 12 & $9-8.6259186 \mathrm{E} 01$ \\
\hline 12 & $12-7.5017975 E$ O1 & 12 & $131.4970625 \mathrm{E} 02$ & 13 & $1-4.8762445 E 00$ & 13 & $22.7884445 E 00$ \\
\hline 13 & $34.9725193 \mathrm{E}-01$ & 13 & $47.6581772 \mathrm{E} 01$ & 13 & $5-6.2984949 E-01$ & 13 & $64.1961641 E 00$ \\
\hline 13 & $73.6651230 \mathrm{E}$ O1 & 13 & $8-4.0041618 \mathrm{E} 00$ & 13 & $97.6815918 \mathrm{E} 01$ & 13 & $13-1.1323302 \mathrm{E} \quad 02$ \\
\hline
\end{tabular}

The time response calculations were performed using the computer code MATEXP. (6) The input to MATEXP consists of the A matri $x$ and $\bar{f}_{d}$ vectors, as defined in Equation (1.6.2).

Results of the frequency response and time response calculation at 100 percent load point are shown in Figures 1.4 to $\mathrm{I} .23$ for the following inputs: helium flow, helium inlet temperature, and feedwater flow. The five runs were labeled in Table 1.4

The selected outputs to be plotted for each run were: (a) main steam outlet temperature (TS4), (b) Helium outlet node average temperature (TGIB), and (c) main stean, outlet average pressure at node $3(\mathrm{~PB})$. 
TABLE I.4

DESCRIPTION OF EACH ANALYSIS RUN

\begin{tabular}{ccc} 
Run No. & $\begin{array}{c}\text { Description of Perturbed } \\
\text { Input Variable }\end{array}$ & $\begin{array}{c}\text { Constant Flow Model or } \\
\text { Constant Pressure Model }\end{array}$ \\
\hline 1 & Helium flow rate $(1 \mathrm{lb} / \mathrm{sec})$ & CFSG \\
2 & Helium flow rate $(1 \mathrm{lb} / \mathrm{sec})$ & CPSG \\
3 & Helium inlet cemperature $\left(i^{\circ} \mathrm{F}\right)$ & CFSG \\
4 & Helium inlet temperature $\left(1^{\circ} \mathrm{F}\right)$ & CPSG \\
5 & Feedwater fion rate $(1 \mathrm{lb} / \mathrm{sec})$ & CFSG \\
\hline
\end{tabular}

1.6.) Discussion of Results for Run No. 1 (Figure 1.4 to I.7).

Run No. I shows the CFSG model time response and frequency response for a $1 \mathrm{lb} / \mathrm{sec}$ increase in helium flow rate. Increased helium flow rate increases the heat transfer rate from the helium to the secondary coolant through the tube wall. For constant outlet steam header pressure and feedwater flow rate, the steam pressure initially rises and then approaches the original value. This is because there is no increase in feedwater flow to sustain the rise. The main steam temperature (TS4) increases significantly $\left(1.44^{\circ} \mathrm{F}\right)$, whereas the temperature rise for the helium outlet node (average) is relatively low $\left(0.63^{\circ} \mathrm{F}\right)$. The steady values of each output in time response after $300 \mathrm{sec}$ are close to the magnitude of the frequency response at low frequency $(0.004 \mathrm{rad} / \mathrm{sec})$ just as they should be. 

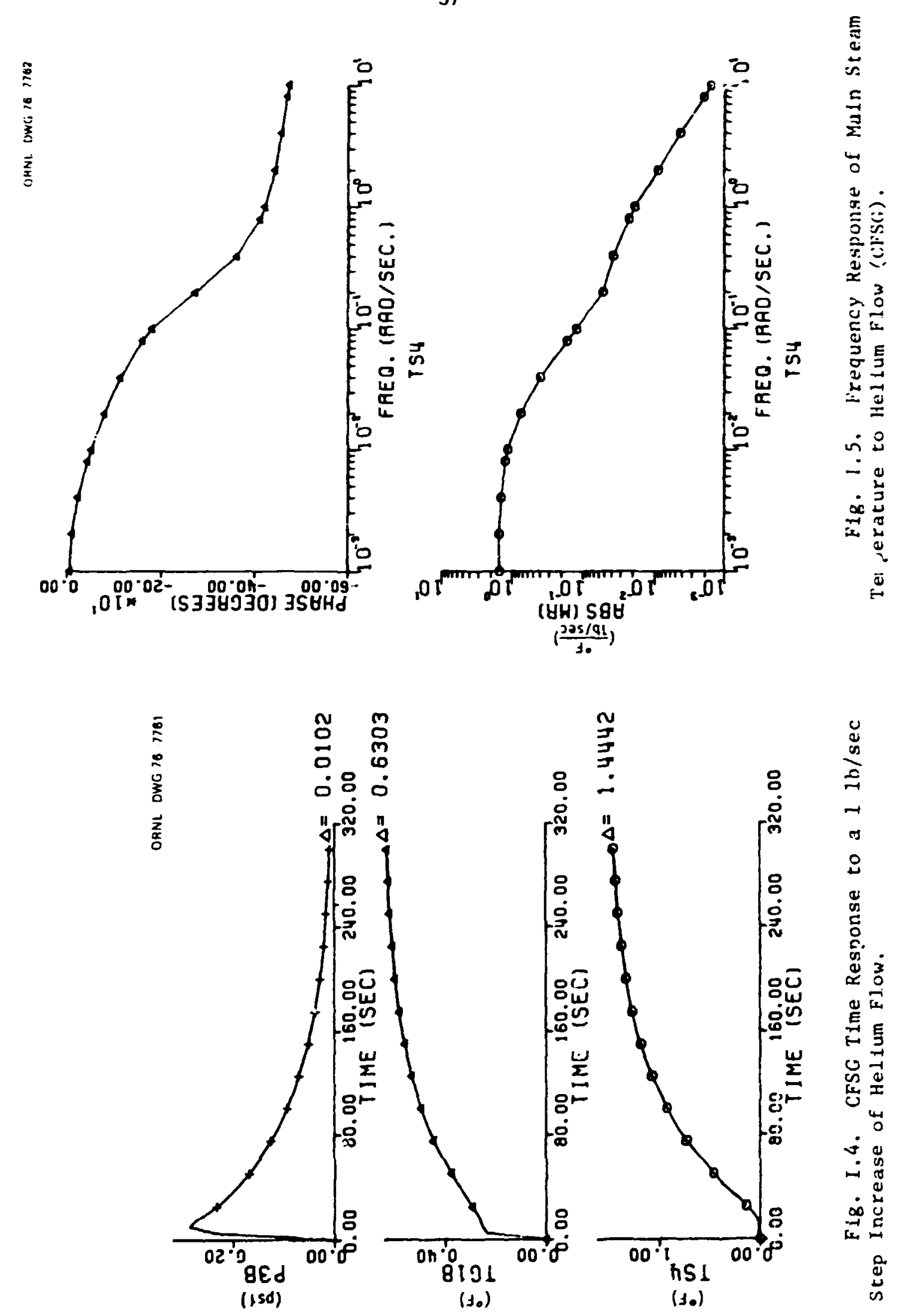
OANL OWG 701764
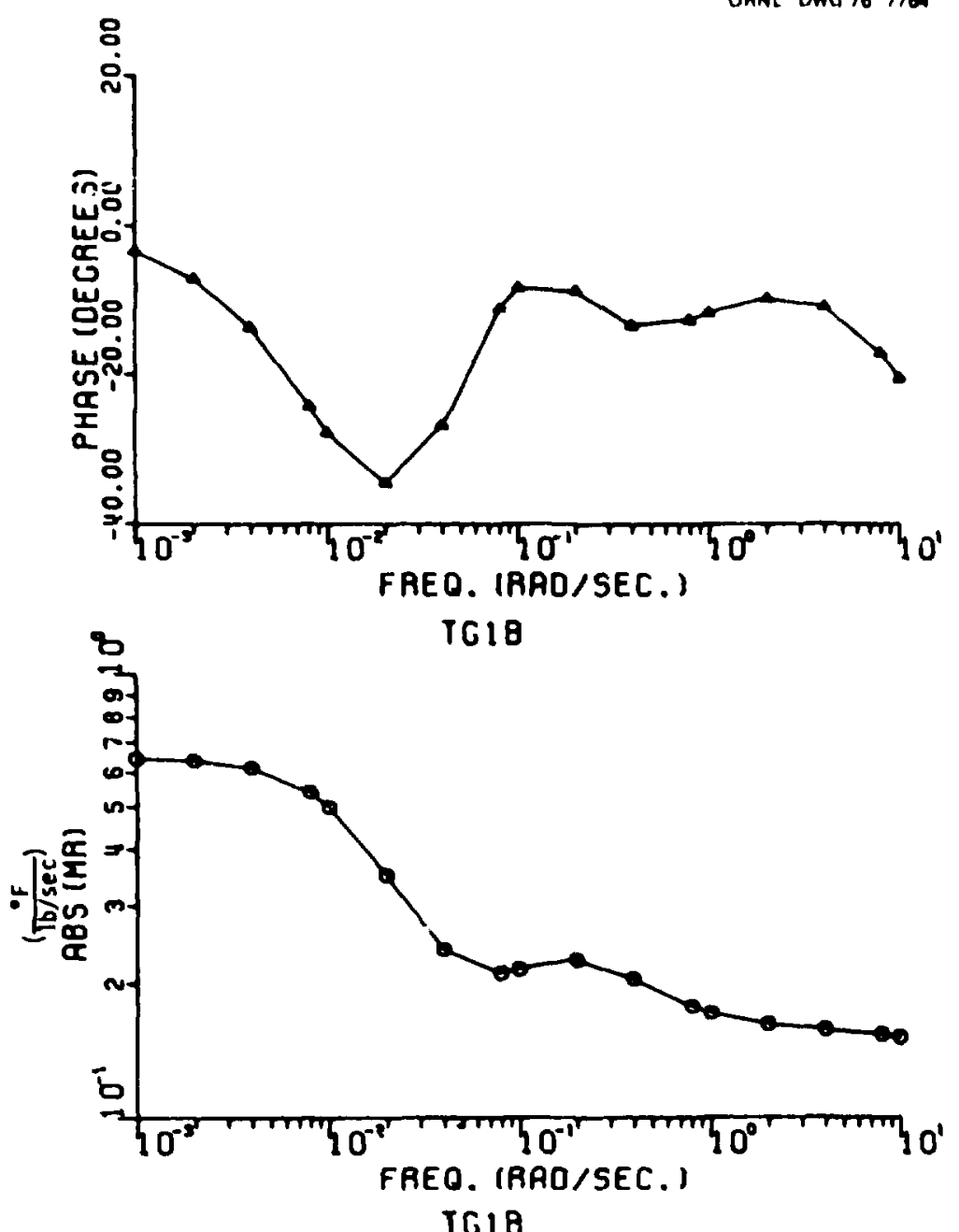

Fig. I.6. Frequency Response of Hellum Outlet Temperature to Hellum Flow (CFSG).
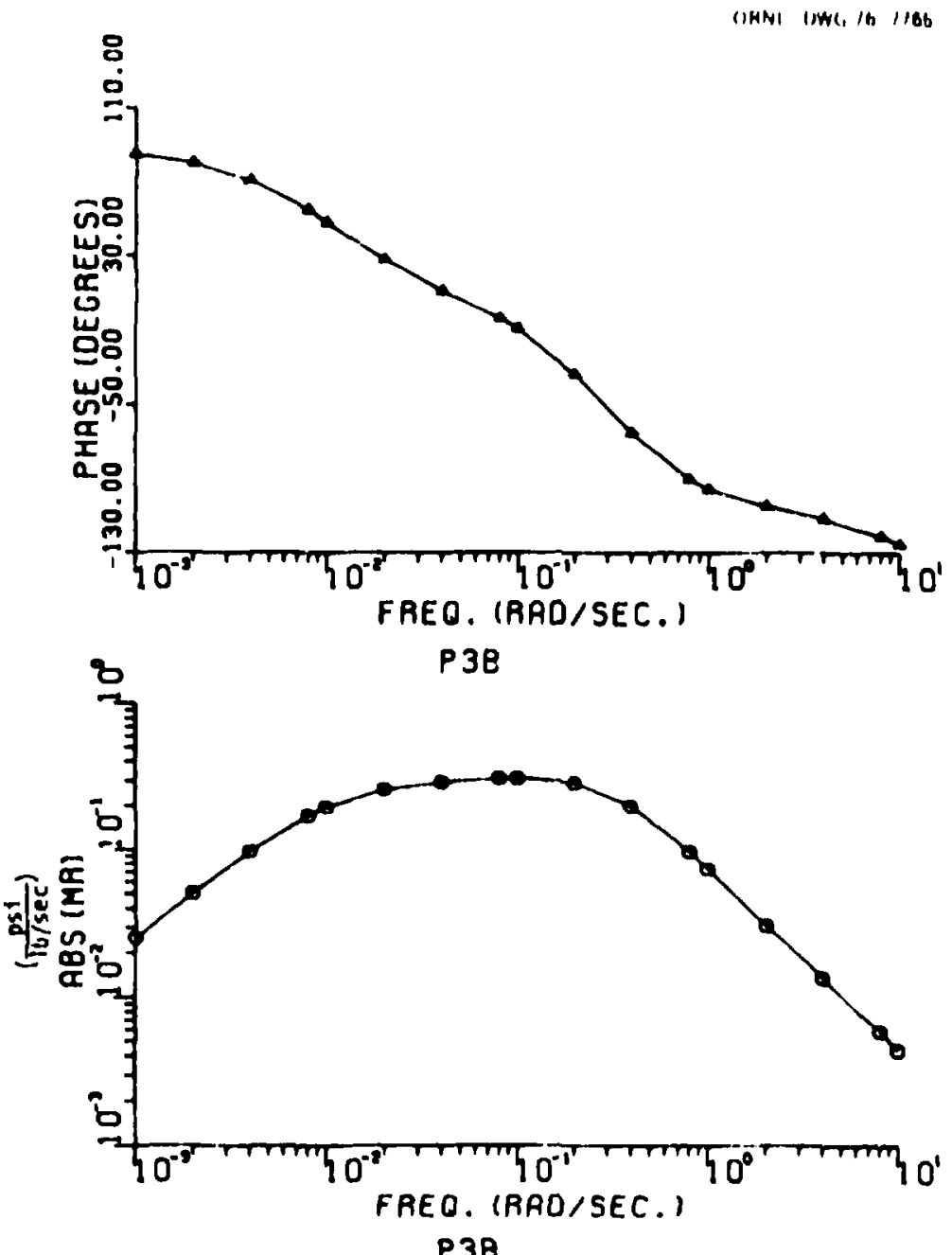

F18. 1.7. Frequency Response of Main Steam Pressure to Hellum Flow (CFSG). 
The response curves of the sutputs are almost monotonic and settle down within 165 seconds $(0.006 \mathrm{rad} / \mathrm{sec}$ in frequency domain). The time lag is primarily due to the themal capacitance of the metal, water/steam mass and the thermal resistance from the primary to the secondary coolant.

I.6.2 Discussion of Results for Run Ho. 2 (Figure I.8 to I.11)

Run lis. 2 shows the CPSG model time response and frequency response for a $1 \mathrm{lb} / \mathrm{sec}$ increase in helium flow rate. The systen
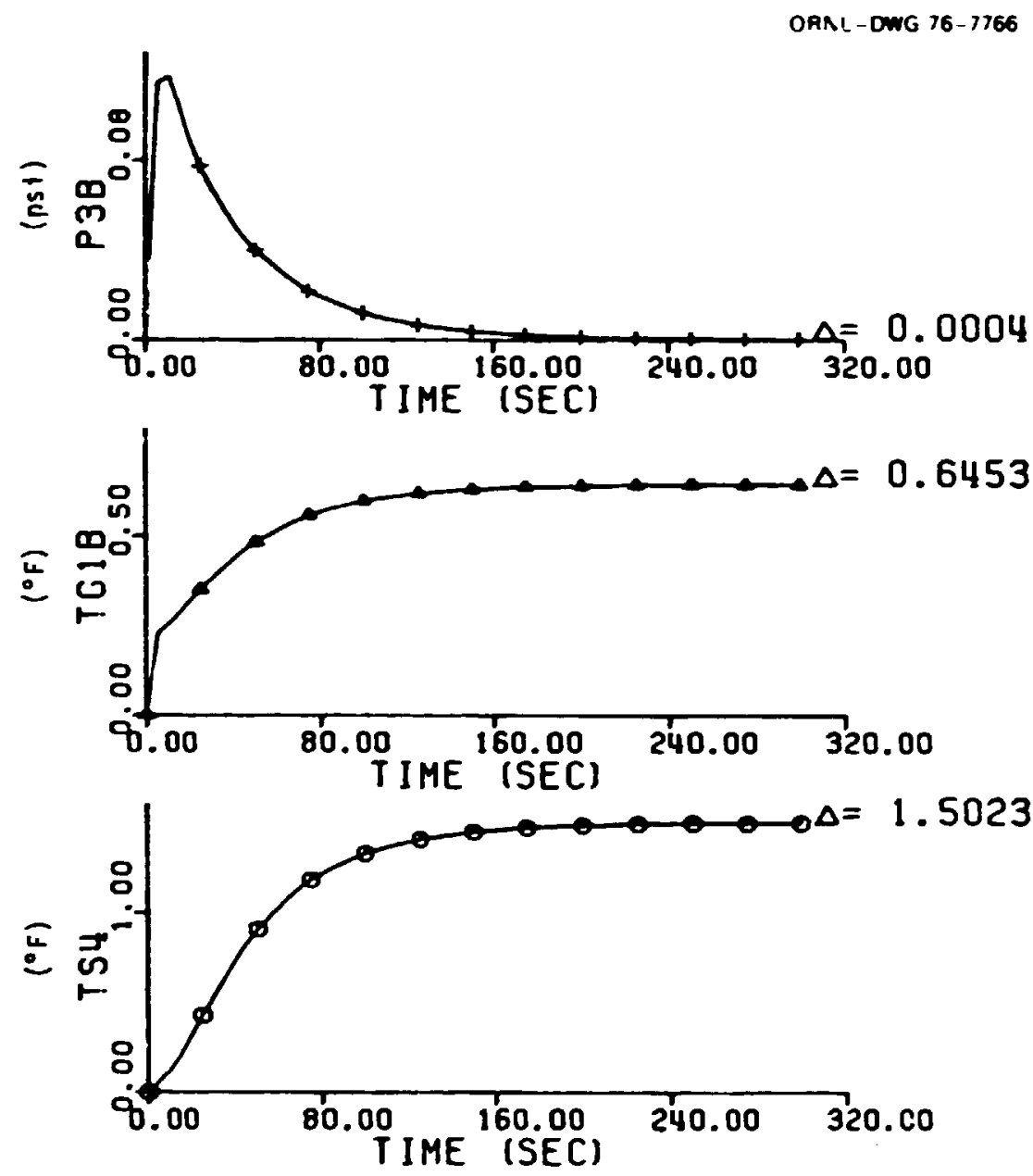

Fig. I.8. CPSG Time Response to a 1 1b/sec Step Increase of Helium Flow. 

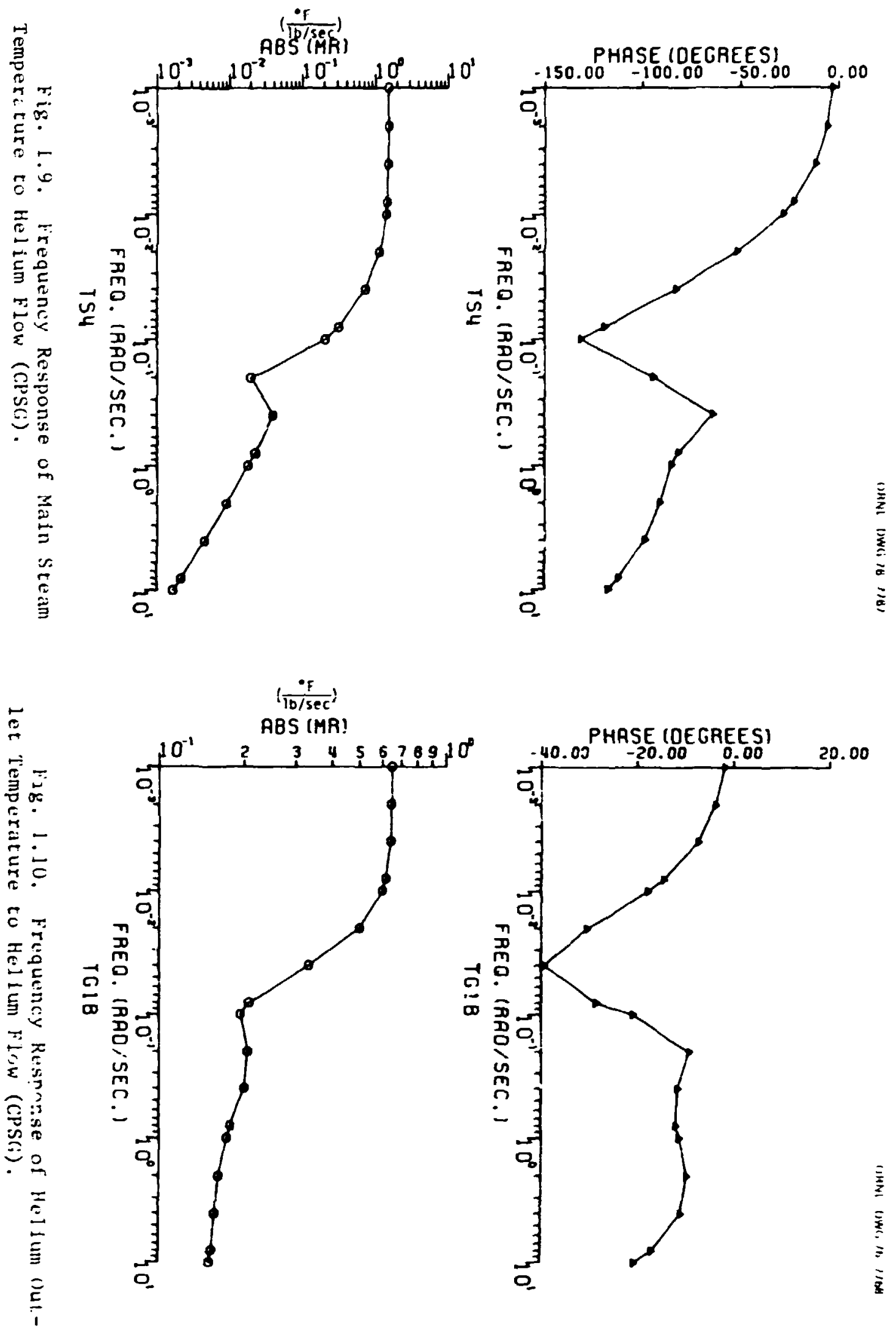

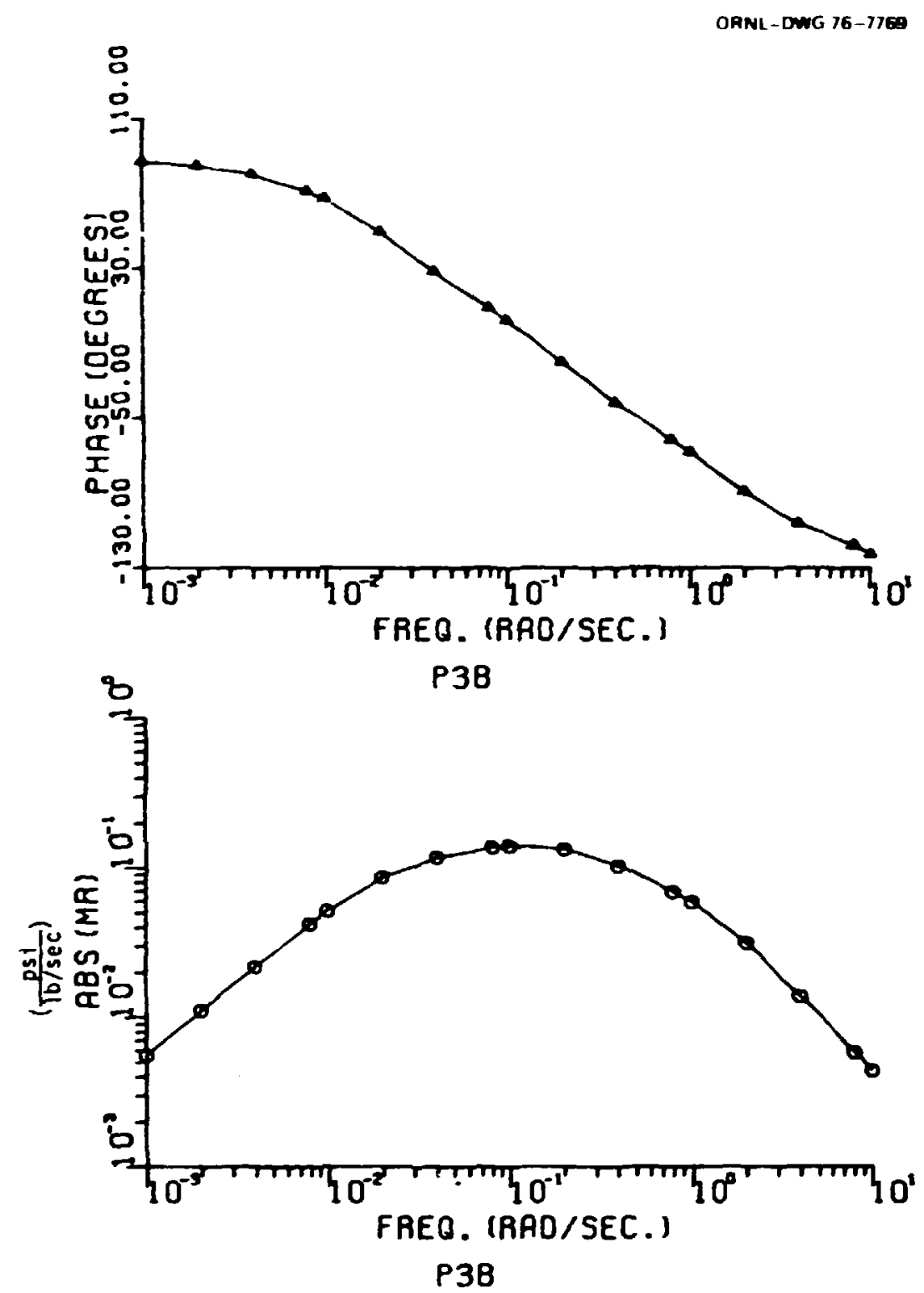

Fig. I.11. Frequency Response of Main Steam Pressure to Helium Flow (CPSG).

responses for "P3B" and "TG1B" are similar to those of Run Ho. 1 (CFSG). There is large difference in the early transient of "TS4" response. For the CPSG mode1, the fast rise of main steam pressure (P3B) reduces the feedwater flow rate which makes the response of "TS4" faster than for the CFSG model in Run No. 1. 


\section{i.6.3 Ũiscussion of Results for Run No. 3 (Figure I.12 to I.15)}

Run No. 3 shows the CFSG model time response and frequency response for a $1^{\circ} \mathrm{F}$ increase in helium inlet temperature. Increased temperature of the primary helium coolant increases the rate of transfer of thermal energy from the primary to the secondary coolant. "P3B" increases initially, ther returns to the original value due to the constant
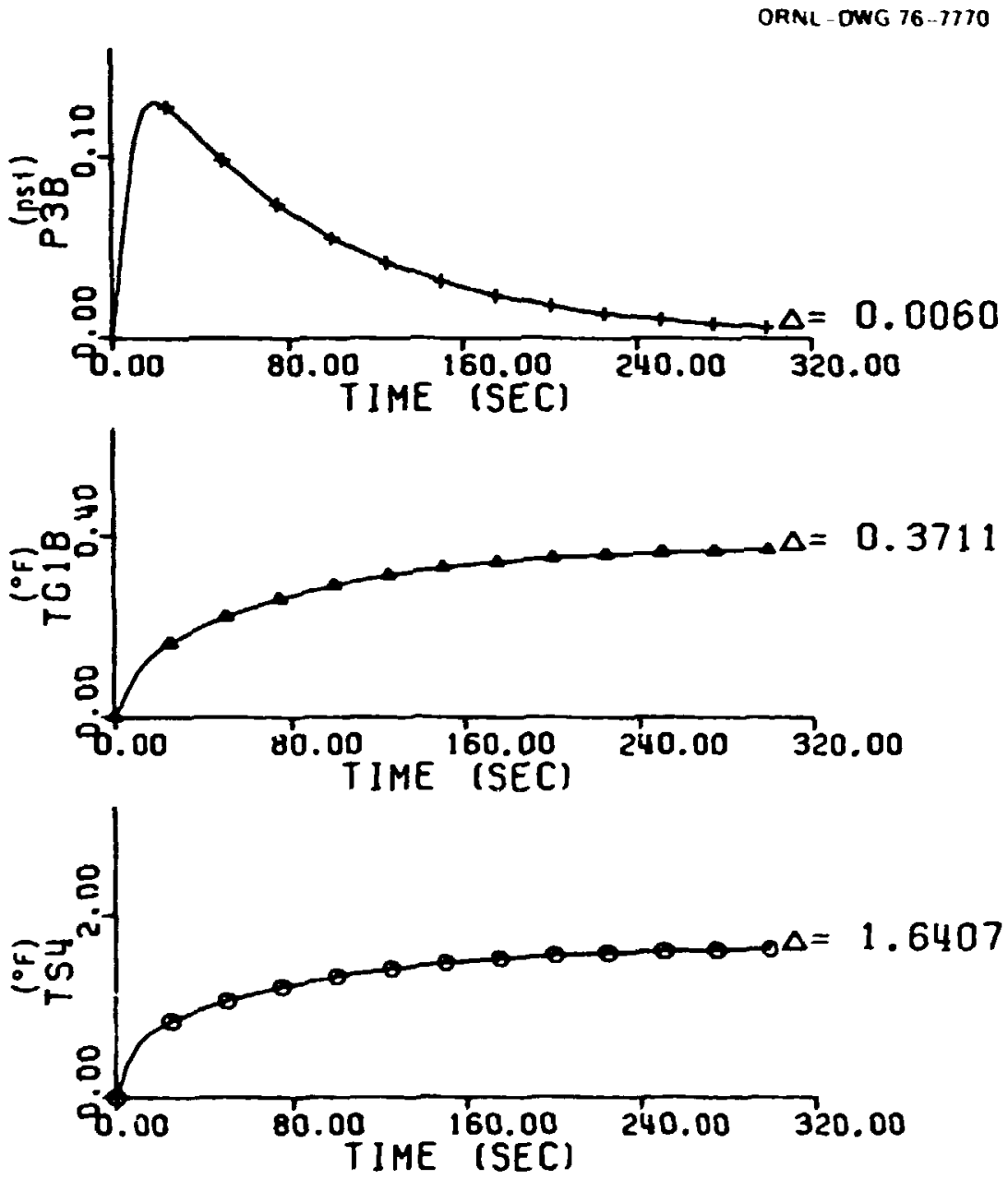

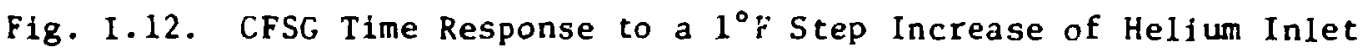
Temperature. 

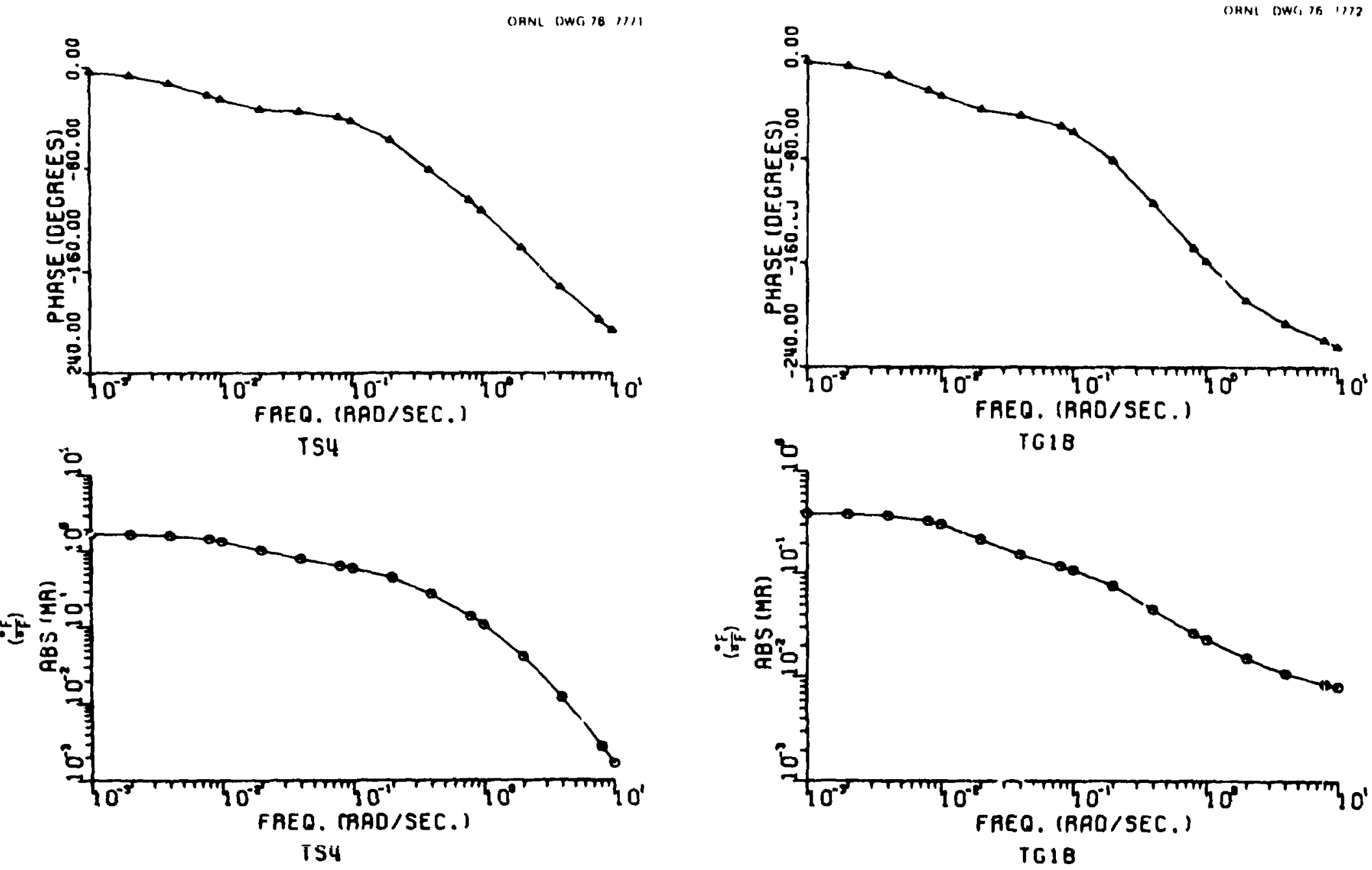

$\vec{\omega}$

F18. I.13. Frequency Response of Maln Temperature to Helfum Inlet Temperature (CFSG).

Fig. 1.14. Frequency Response of Hellum Outlet Temperature to Hollum Inlet Temperature (CFs). 

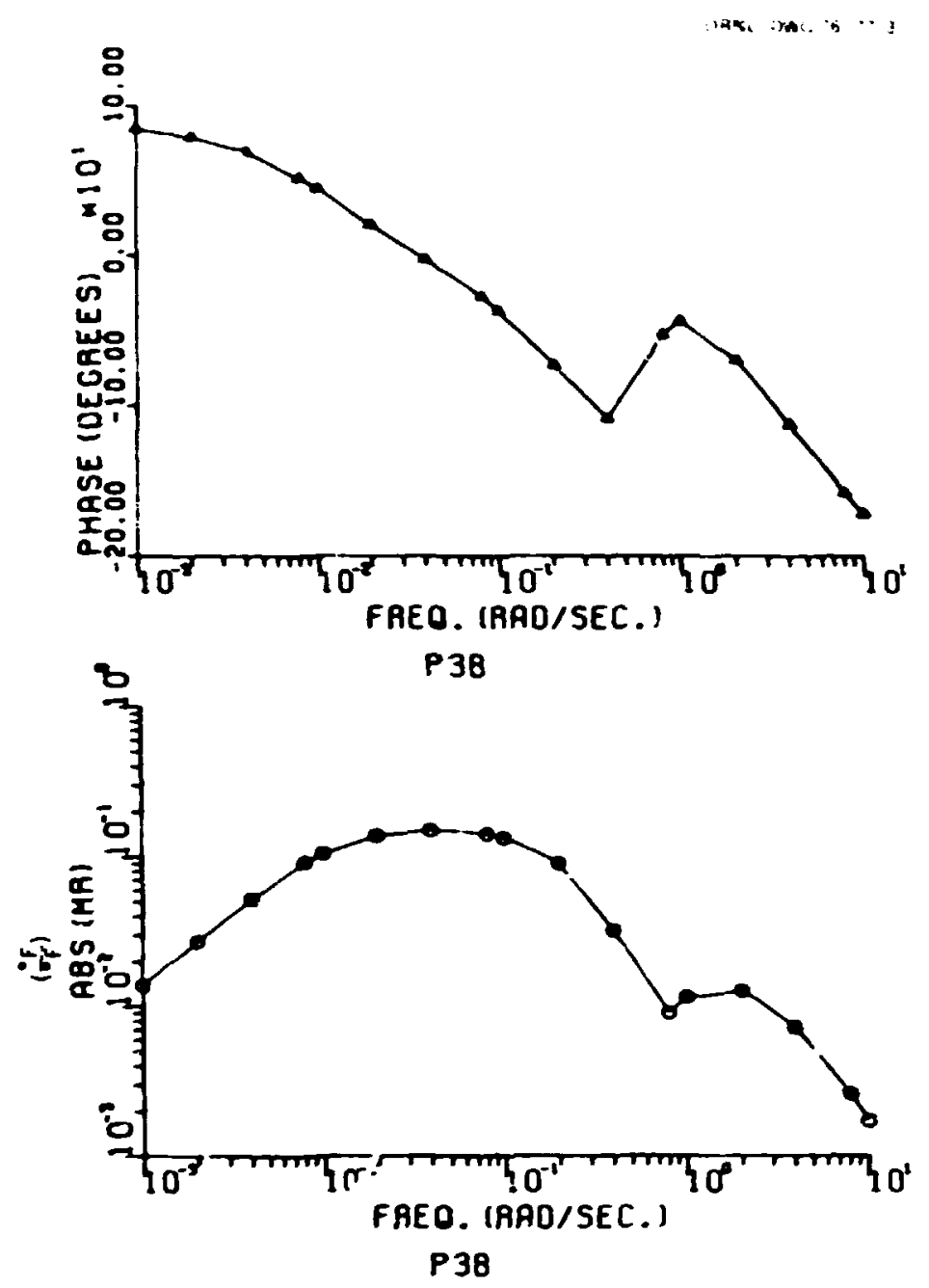

Fig. I.15. Frequency Response of Main Steam Temperature to Helium Inlet Temperature.

feedwater flow. All the temperature nodes increased following the increase of helium inlet temperacure.

1.6.4 Discussion of Results for Run No. 4 (Figure I.16 to I.19). Run No. 4 shows the CPSG model time response aild frequency response for a $l^{\circ} \mathrm{F}$ increase in helium inlet temperature. The system transient responses for this run are similar to those of Run No. 3. 
OANL DWG $76 \quad 774$
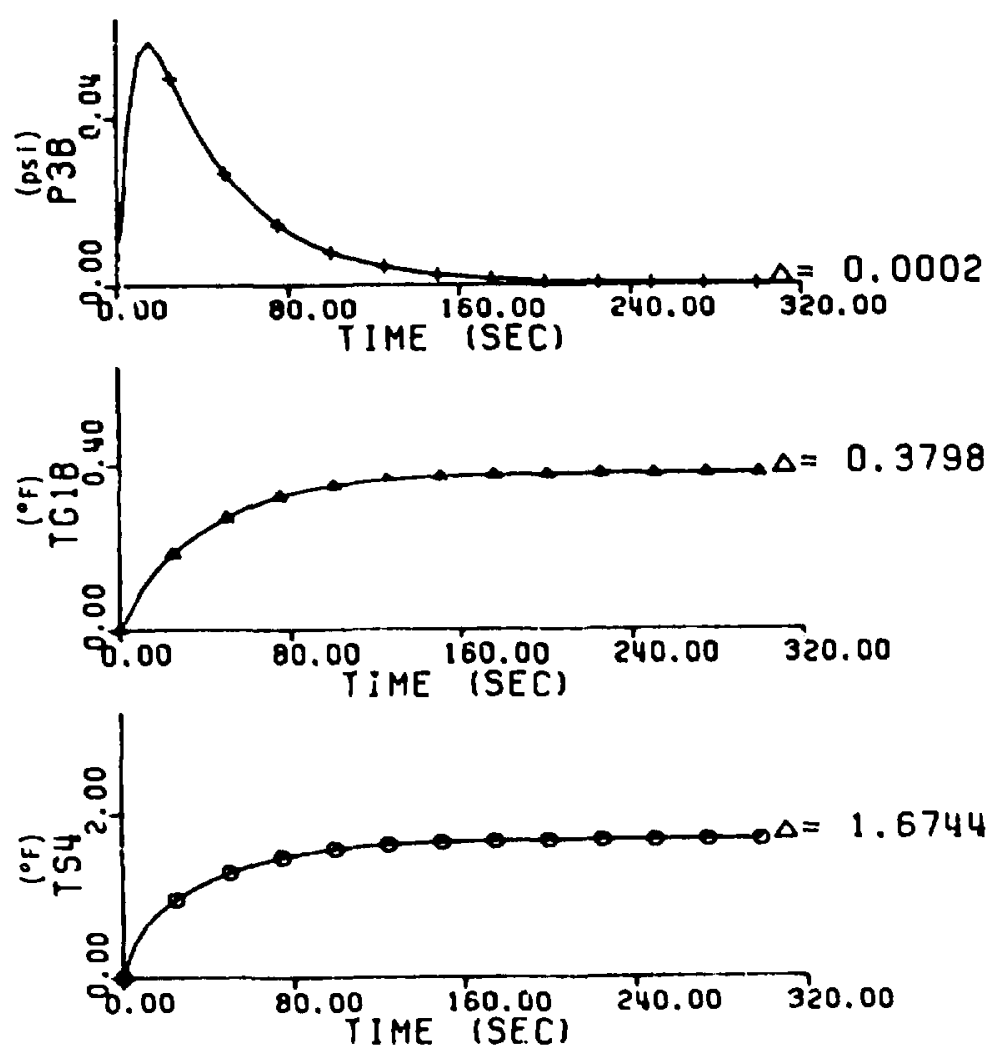

Fig. I. 16. CPSG Time Response to a $1^{\circ} \mathrm{F}$ step Increase of Helium Inlet Temperature.

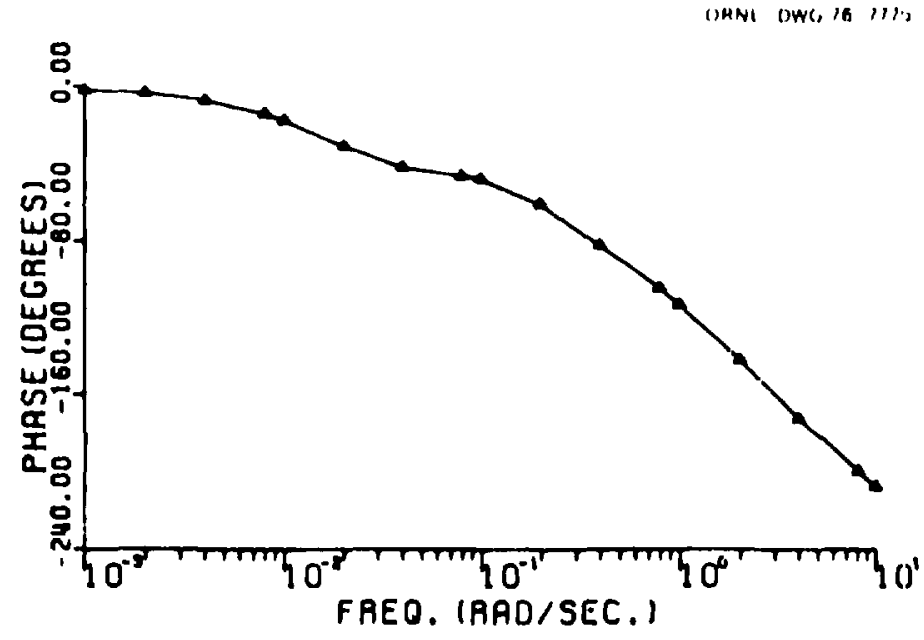

TS4

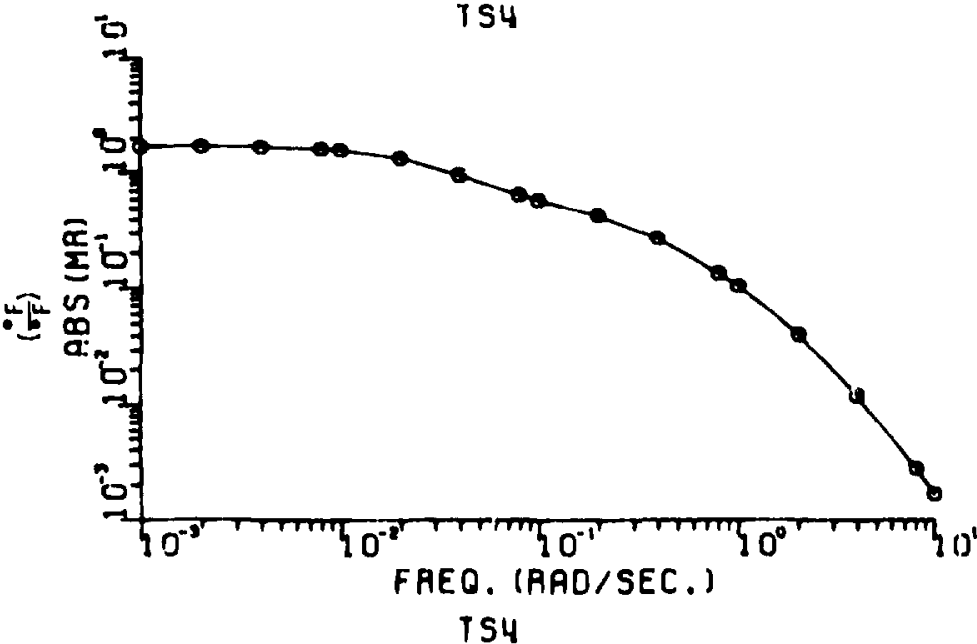

H1g. I.17. Frequency Response of Maln Stean lemperature to Hel lum Inlet Temperature (CPSG). 

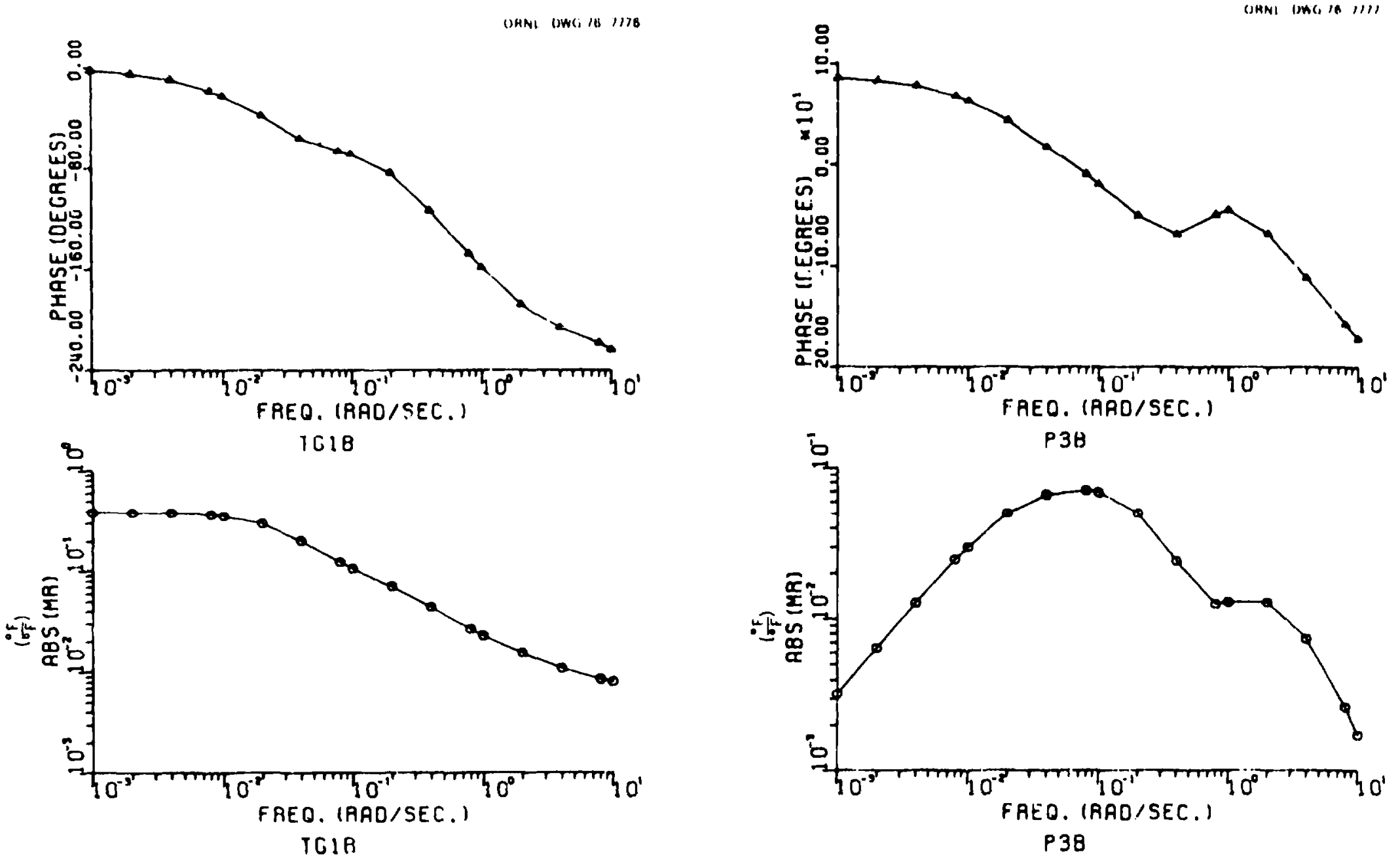

Fig. 1.18. Frequency Response of Helium outlet Temperature to Helium Inlet Temperature.

Fig. 1.19. Frequency Response of Main Steam pressure to Hellum Inlet Temperature (CPSi). 
1.6.5 Ũiscussion of Resuits för Rün Ho. 5 (figute !.2n te I.23). Run No. 5 shows the CFSG time response and frequency response for a $11 \mathrm{~b} / \mathrm{sec}$ increase in feedwater flow rate. For constant helium flow rate and inlet teriperature, the temperature in the secondary coolant circuit drops $\left(-2.74^{\circ} \mathrm{F}\right)$ following the increased feecwater rlow rate. The helium outlet temperature also drops $\left(-0.60^{\circ} \mathrm{F}\right)$. For constant outlet steam header pressure, the main steam pressure increases by 0.71 psi in order to sustain the increase in the feedwater flow rate.
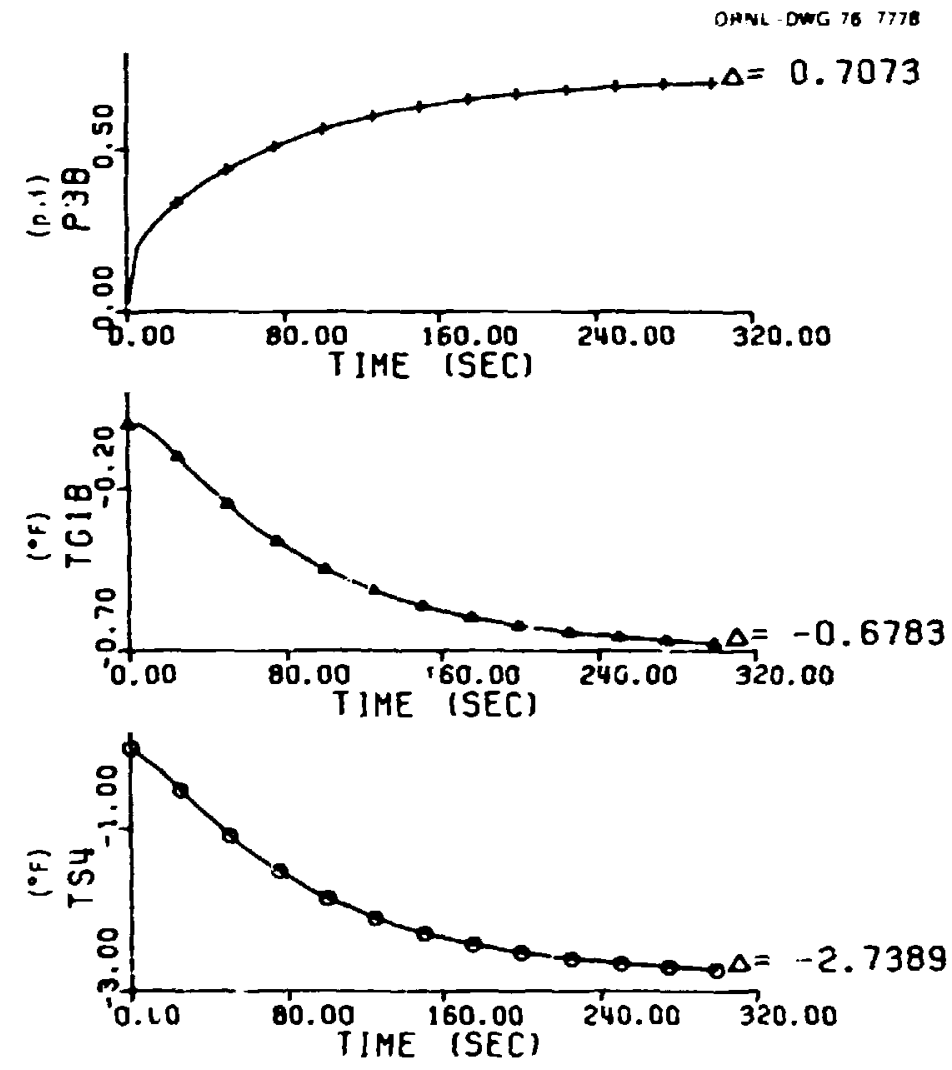

rig. I.20. CFSG Time iesponse to a $11 \mathrm{~b} / \mathrm{sec}$ Step Increase of Feedwater Flow. 

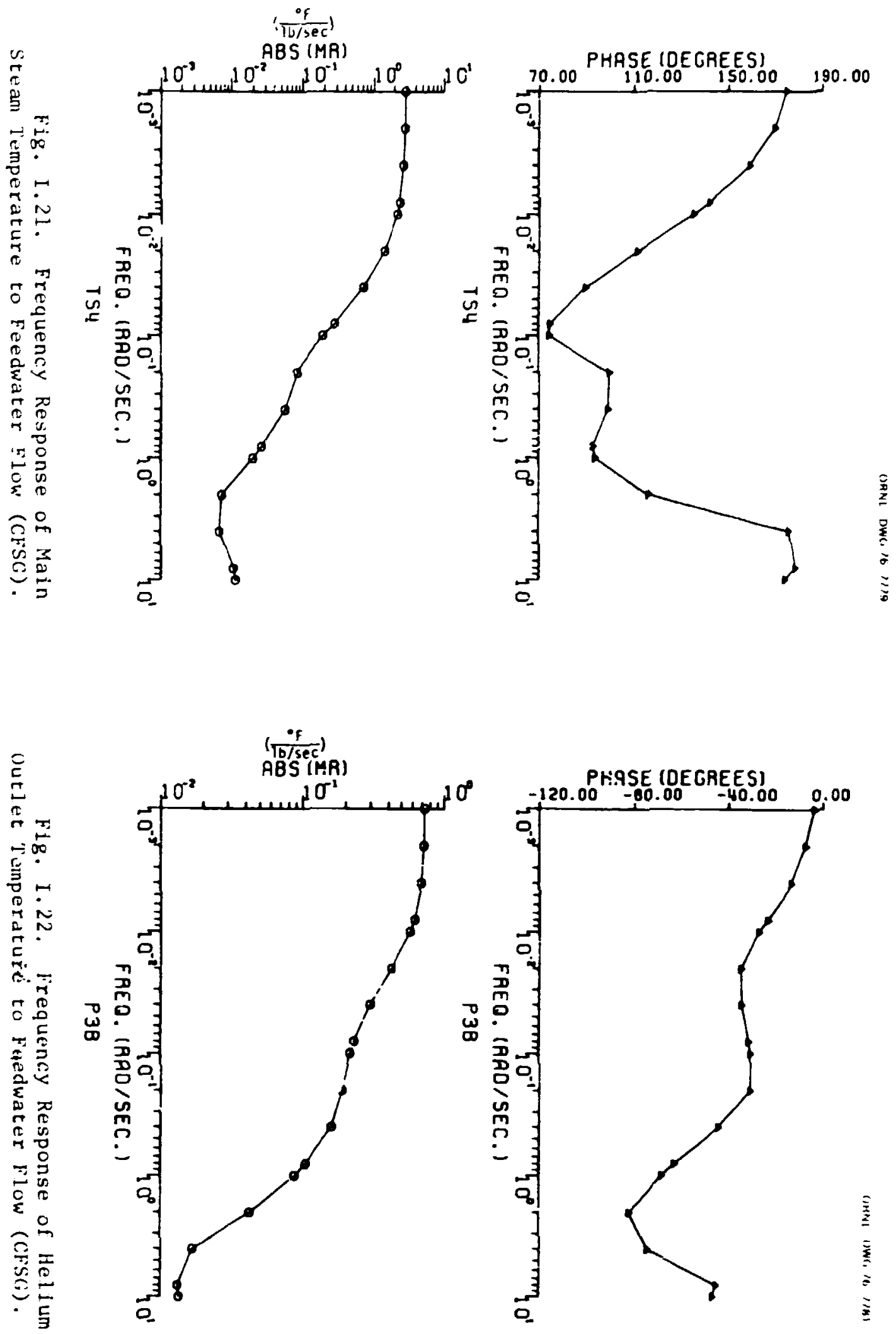

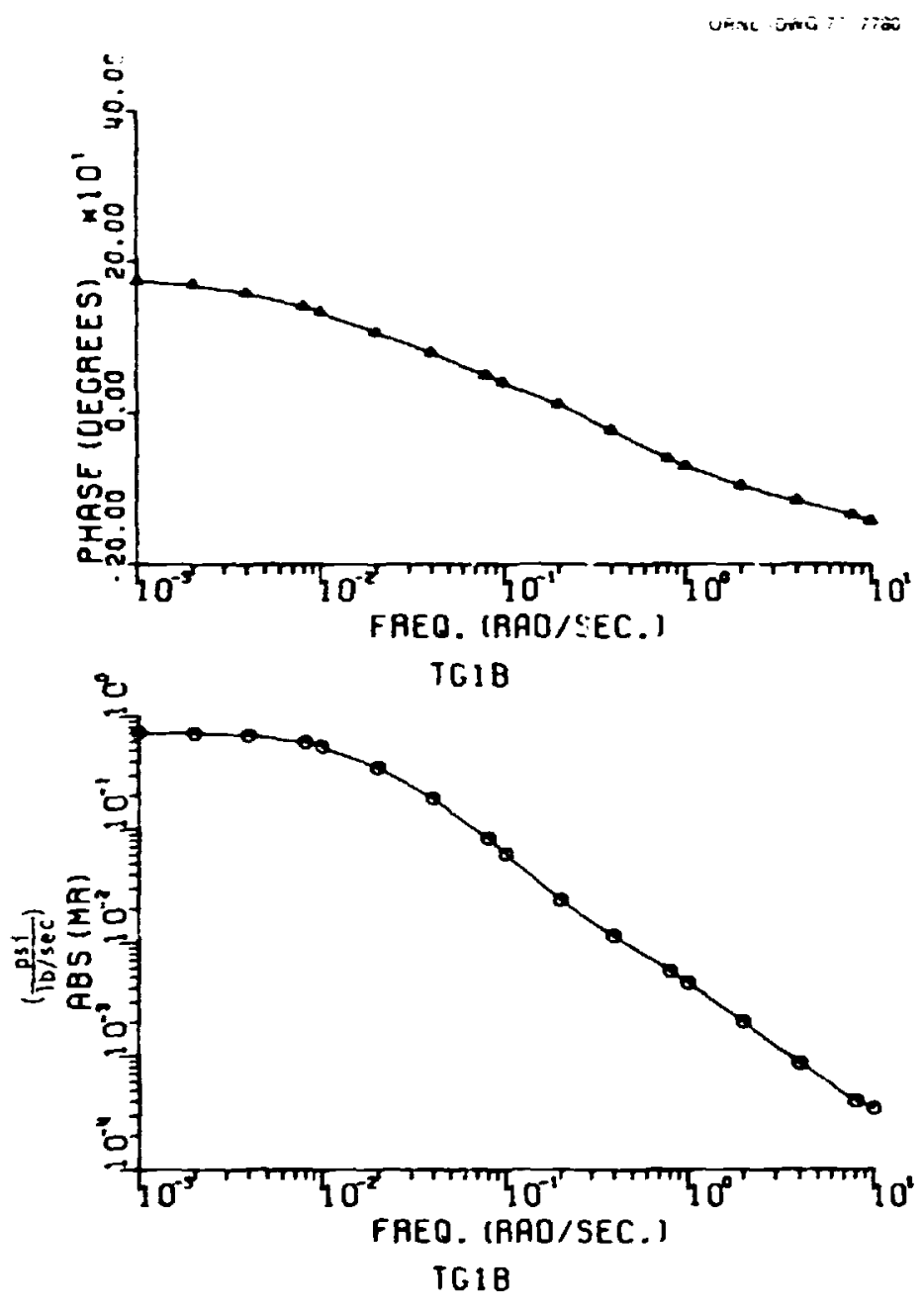
(CFSG).

Fig. I.23. Frequency response of Main Steam Pressure to Feedwater Flow References

1. Richardson, D. C., "Linear Modeling and Control of High Temperature Gas-Cosled Reactor (HTGR) Power Plant," Gulf-GA-Al2062, April 1972.

2. Savery, C. W., et al., "TAP - A Fortran IV Program for tne Transient Analys is of the HTGR Power Plant Performance," Gulf General Atomi c, 1966. 
3. Leech, H. D., Personal communication, General Atomic, 1974.

4. Ray, A., "A Nonlinear Dynamic Model of a Once-Through Subcritical Steam Generator," M. S. Thesis, Northeastern University, 1974.

5. Harrington, R. M., Personal communication, The University of Tennessee, 1974.

6. Ball, S. J. and R. K. Adams, "MATEXP: A General Purpose Digital Computer Program for Solving Ordinary Differential Equations by the Matrix Exponential Method," ORNL-TM-1933, Oak Ridge National Laboratory (August 1967). 
CHAPTER I!

NONL INEAR MODELING OF A "'GR STEAM GENERATOR

Ming-Huei Lee

A nonlinear model is important wher large disturbanzes or various operation levels are involved in the simulation of a steam generator. It is expected to be more accurate than the linear model for these concitions. The nonlinearity of the system parameters and the spatial effects cause the problem to be quite complex, Moreover, since the unrertainties in the system parameters are significant, the results will have uncertainties. Therefore, in the noniinear model, the spatial effect, the nonlinear parameters and the uncertainties in system parameters are three major areas of concern.

Practical methods for handling spatial effects require the use of lumps to represent the spatial distributions. However, as the number of lumps is increased, the computing time is also increased. It is important to know whether a few-lump model can provide the necessary accuracy. In this study, the number of lumps will be varied to find the number required to reach asymototic performance as the number of lumps increases.

The system parameters which are involved in major nonlinear terms include the therma? conductivities, heat capacities, and viscosities of iluid (steam, water, helium) and/or tube metal, surface tension of water, supression factor and some other factors in the heat transfer correlations. These parameters may be evaluated using available subroutine programs or by using emperical equations which are obtained by least square methods. 
In studying the uncertainties iñ systen responses due to parameter uncertainties, the heat transfer coefficients and two phase multiplier factors are most importunt, since their inaccuracy could be up to 0.0 . 0ther important parameters with significant uncertainties will also be studied for their sensitivities.

The dynamic equations for the transient state were derived from the fundamental differential equations. The conservation of energy, the conservation of momentum, and the conservation of mass equations were developed into a moving boundary model. The developed model was compared to different models from a literature survey so that an assessment of model assumptions and approximations could be made.

The literature was revi wed to find the data and correlations required for the steam generator model. A short summary of the information from the literature and other preparations for the model development follow:

A. Survey on heat transfer correlations and/or friction factors.

1. For single phase

a. Seban and McLaughlin's (l) correlation

b. Mori and Nakayama's (2) correlation

c. Pressure Drop and Heat Transfer in Coils summarized by Srinivasan et al. (3)

d. Srinivasan's ${ }^{(4)}$ correlation e. Ito's ${ }^{(5)}$ correlation.

All of the above correlations are for helical coils one of the features of the HTGR steam generator. The correction factor for helical tubes from (e) or (a) was 
seierted to use in this stuudy, since the applicable range of Reynold numbers and curvature is adequate for HTGR steam generator.

2. For two phase, steam/water

a. Borishanskij, et al's. (6) correlation (straight tube)

b. Owhadi, Bell, and Crain's (7) correlation (coil tube)

c. Crain and Beill's ${ }^{(8)}$ correlation (coil tube)

d. Chen's ${ }^{(9)}$ correlation (straight tube)

e. Rohsenow's $(10,11)$ correlation (straight tube)

f. Miropolskii's (12) correlation (straight tube)

(a) through (e) are for nucleate boiling and subcooled boiling. (f) is for film boiling.

Chen's correlation, Rosenhow's correlation, and Borishanskij's corrections were selected for use in the nucleate boiling and subcooled boiling region. The last two correlations were evaluated at high pressure and high temperature. The Miropolskiy's correlation was selected to use in the dryout region for high press:ure and high temperature. The correction factor for helical coils is the same as for single phase, which is based on the discussion in (b) or the suggestion of Collier.

3. For single phase, steam

a. Miropolskii and Shitsman ${ }^{(13)}$ correlation

b. Bishop et al's. (14) correlation

The correction factor for coil tube is the same as for single phase water. 
4. For helium side heat transfer and pressure drop through banks of helical tubes, the Gilli's $(15)$ correlations were selected to use. Grimicon's (16) straight tube bank data are necessary for evaluating Gilli's ccrrelations.

Development of the two phase pressure gradient in homogeneous models by employing colebrook (17) friction coefficient, Ito's curve pipe correction factor, and IfcAdams' $(9,18)$ two phase viscosity's definition was made.

B. Obtaining the emperical equations for the nonlinear parameters. The thermal conductivities of the fluid and tube metal, the viscosities of fluid, the surface tension of water, suppression factor and so on are necessary to obtain their emperical equations for practical use. Some of these equations may be found in the literature survey; some of them were obtained by using linear regression methods to fit the numerical table:

C. Steady State Program.

The steady state program has been developed in the multilump case so that the steady state distributions of the state variables can be obtained. This was done for three purposes: (a) To study the differerces of the results by using different correlations and to compare them to the HTGR steam generator design data; (b) to evaluate the adequate average state variables for the transient study in a few lump models; (c) to provide the initial conditions to the transient case. 


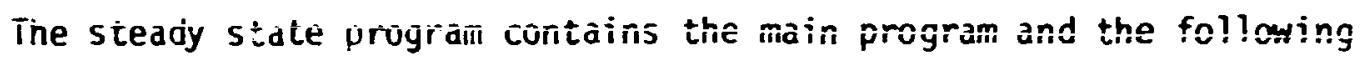
subroutines:

1) SUBROUTIINE FRCTH

2) SUBROUTINE THOFRR.

3) SUBROUTINE SUBFRC

4) SUBROUTINE EQCKT

5) SUBROUTINE TWOEKI

6) SUBROUTINE TWOEK2

7) SUBROUTINE SUBEK

8) SUBROUTINE VSCST

9) SUBROUTINE VISFG

10) SUBROUTINE SUBVIS.

1), 2), and 3! are used to evaluate the Colebrook friction factors by an icoration method for single phase steam, two phase steam/ water and single phase water respectively. 4j, $, 1,6$, and 7) are used to evaluate the equivalent thermal conductances, tube temperature and tube surface temperature by an iteration method for single phase steam, two phase steam/water dryout region, two phase steam/water nucleate boiling region, and the single phase water region respectively. 8), 9), and 10) are used to evaluate the dynamic viscosities of the above three regions. The steady state program is now being tested and debugged.

In the future, the following are planned:

1. Comparison of steam generator models using different assumptions, approximations, and correlations.

2. Steam generator dynamic simulation. 
3. Sensitivity to the system parameters.

4. Study of small and large disturbances.

5. Extension of the model from a few lumps to many lumps.

Reíerences

1. R. A. Seban and E. F. McLaughlin, "Heat Transfer in Tube Coils with Laminar and Turbulent Flow," Int. J. Heat Mass Transfer, Vol. 6, pp. 387-395, 1963.

2. Yasuo Mori and Wataru ilakayama, "Study on roriced Convective Heat Transfer in Curveo Pipes," Int. J. Heat Ma:s Transfer, Voi. 10, pp. 681--695, 1967.

3. P. S. Srinivasan, S. S. Nandapurkar, and F. A. Holland, "Pressure Drop and Heat Transfer in Coils," The Chemical Engineer, London, 1968, Wo. 218, P.CE113.

4. P.S. Srinivasan, S. S. Nandapurkar, and F. A. Holland, "Friction Factors for Coils," Trans. Instn. Chem. Engrs., Vol. 48, p. T156, 1970.

5. H. Ito, "Friction Factors for Turbulent Flow in Curved Pipes," J. of Basic Engineering, p. 123, June 1959.

6. A. A. Andreevskii, V. M. Borishanskii, V. S. Fokin, V. N. Fromzel, G. P. Danilova, G. S. Bykov, and V. A. Chistyakov, "Recommendations for Computing the Heat iransfer Coefficient in a Two-Phase Steam-Water Flow in the Pipes of a Steam Gerierator," Heat Transfer-Soviet Research, Vol. 5, Ho. 3, p. $130,1973$.

7. Ali Owkadi, K. J. Bell, and B. Crain, "Forced Convection Boiling Inside He ically-Coiled Tubes," Int. J. Heat Mass Transfer, Vol. 11, pp. 1779-1793, 1968.

8. B. Crain and K. J. Bell, "Forced Convection Heat Transfer to a Two-Phase Mixture: of Water and Steam in a Helical Coil," American Institute of Chemical Engineers Symposium Series, No. 131, Vol. 69, p. 30, 1973.

9. J. G. Collier, Convective Boiling and Condensation, McGraw-Hill Book Company, 1972. 
10. W. M. Rohsenow, "A Method of Correlating rieat-Transfer Data for Surface boiling of liquids," Trans. of AS!AE, Vol . 74, pp. 968-976 (1952).

11. L. Duchatelle, L. de Nucheze, and M. G. Robin, "Theoretical and Experimental Study of Phenix Steam Generator Prototype Modules," iluclear Technology, Vol. 24, p. 123, 1974.

12. Z. L. Miropoiskii, "Heat Transfer in Film Boiling of a Stean-Water iixture in Steam-Generating Tubes," AEC-tr-6252, 1963

(Jeploenergotika, Vol. 10, No. 5, p. 49, 1963).

13. L. Miropolskii and M. E. Shitsman, "Heat Transfer to Water and Steam at Variable Specific Heat (in near critical region), "Soviet Physics-Technical Physio, Vo1. 2, No. 10, p. $2196,1957$.

i4. A. A. Bishop, F. J. Krambeck, and R. 0. Sanderg, "High Temperature Supercritical Pressidre Water Loop, Part III, Forced Correction Heat Transfer to iuperheated Steam at High Prossure and $\mathrm{High}$ Prandtl Numbers," WCAP-2056 (pt. 3) 1964.

15. P. V. Gilli, "Heat T:-ansfer and Pressure Drop for Cross Flow Through Banks of Multiscart Helical Tubes with Uniform Inclinations and Uniform Longitudinal Pitches," Nuclear Science and Engineering, 22, pp. 298-314 (1965).

16. E. D. Grimison, "Correlation and Utilization of New Data on Flow Resistance and Heat Transfer for Cross Flow of Gases Over Tube Banks," Transactions of the American Society of Mechanical Engineers, Vol. 59, p. 583, 1937.

17. L. F. Moody, "Friction Factors for Pipe Flow," Transactions of the ASME, Vol. 66, p. $671,1944$.

18. W. M. McAdams, et al., "Vaporization Inside Horizontal Tubes-11Benzene-0il Mixtures," Transactions of the ASME, Vol, 64, p. 193 (1942). 


\section{CHAPTER I II}

GAS-COGLED STEAM GENERHTOK LINEAR MDDEL

David G. Penfro

This phase of the project is concerned with the development and investigation of a linear, moving-boundary model for the fort St. Vrain steam generators. In this progress report, a brief history of the work in this area will be reviewed before describing the current project in greater detail.

General Atomic uses a linear model of the HTGR-IHSS in part of the design process of the plant control system. This matinematical model is Called LAP (Linear Analysis Program). It was developed by combining linear modeis of the reactor, primary coolant, and secondary coolant systems. Each of these models was derived from first principles using the basic conservation equations exceft for the steam generator. GA was unable to develop a satisfactory steam generator model by the time the rest of the system nodels were ready, so an empiricai or transfer function model was substituted. However, a model based on the physical system still was considered to be more desirable. The development and evaluation of this model is the purpose of the current project.

General Atomic developed a lumped-parameter, three-node, movingboundary mcdel for the steam generator. The lumped parameter approach was chosen for its simplicity and ease of solution. The moving boundary tecionique attempts to account for movement of the beginning and end of the boiiing zone as steam generator conditions change. This is accomplished by divining the main steam section into three regions with moving boundaries between the economizer and evaporator zones and the evaporator 
and superheater zones. This representation allows a fairly simple model that overcomes the difficulties encountered by fixed boundary models.

The oroject at The University of Tennessee is based on the criginal GA model just described. A version of LAP and the siecessary data including steady state parameters were supplied by GA. To improve the physical validity of the time responses of the original GA steam generator mode?, S. I. Chang at UT made several basic modifications. These included the revision of equations to allow for replacement of node-boundary state var ables by node-averaged state variables where applicable. A sketch of the stean generator model with variables as modified by Chung is shown in Figure III.1. As indicated by the figure, both node-boundary state

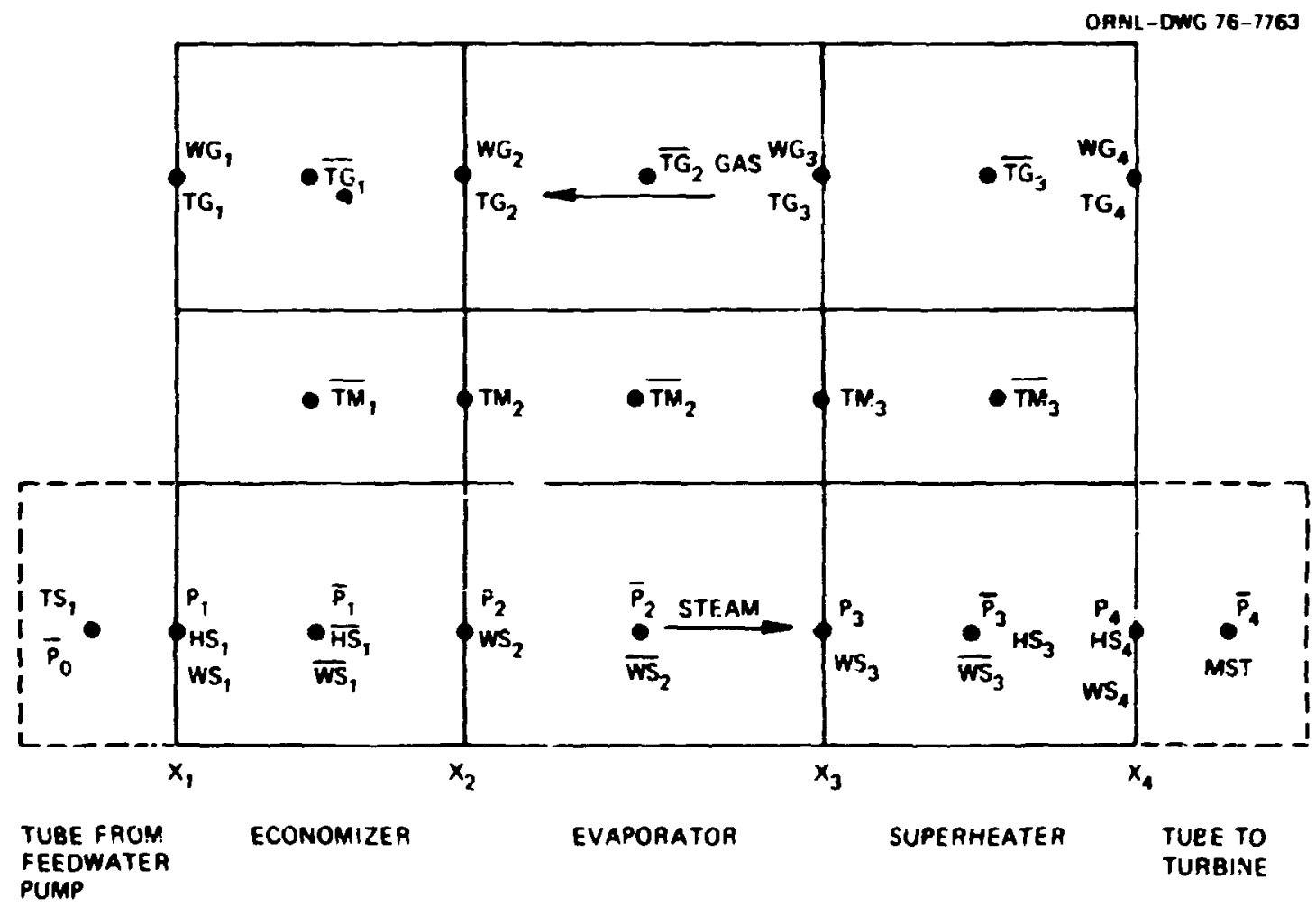

TS: : FEEDWATER TEMPERATURE

MST: MAIN STEAM TEMPERATURE

Fig. III.1. Steam Generator Nodal Structure. 
node-averaged variables remain in the model equations. As suggested by $G A$, these are related by using a weighting factor, $W_{i}$, as follows.

$$
\nabla_{i}=Y_{i} H_{i}+Y_{i+1}\left(1-H_{i}\right) \quad, 0 \leq H_{i} \doteq 1,
$$

where $Y_{i}$ and $Y_{i+1}$ are the boundary quantities and $\bar{Y}_{i}$ is the node-dveraged quantity. The weighting tachnique is very significant since it is used for temperatures on the primary side and for flow rates, pressures, and enthalpies on the secondary side. In addition, internal energies. specific volumes, and temperatures on the secondary side are calculated from the previously-averaged pressures and enthalpies. GA suggested specific vaiues for each of the three nodal weight factors at which they arrived by physical and intuitive reasoning. These suggested weight factors influenced the calculation of the steady state parameters they supplied. Chang used these weight factors and the rest of the GA-supplied data to produce time responses that seemed reasonable and valid. However, when he varied the weight in the helium entrance node, $\left(W_{3}\right)$ to invsstigate its effect, the time responses were similar in character to the previous ones but markedly uifferent in magnitucic. This resu?t was a subject for some concern for at least two reasons. First, the primary side inflisence was not thought to be so significant. Also, $C_{2} A$ arrived at the suggested weight: fairly arbitrarily so there was little reason to believe they were the ideai ones to ise.

This area of concern with weighting factors along with a basic need to verify the model madifications independently were the reasons behind this phase of the project at UT. The initial purposes were to verify the unexpected significant dependicnce on the primary side weighting 
f̂cturs, next to investigate these effects on both the primary and secondary sides, and then to offer some explariation for the dependence or to suggest changes to improve the weighting technique. Since the beginning of this work, the investigation has broadened to include a more general sensitivity study of the mode? and examination of model assumptions made in its development.

The rirst task was to verify the work done on the model at UT by S. I. Chang. An attempt was made to duplicate his development and compare the rosults at each stage. First, the model equations were derived from the basic censervation equations. Next, the equations were linearized and set up in the model form with specified state variables and perturbation variables. All discrepancies found were resolved by mutual agreement and the final model form was decided upon. The next task involved a review of LAP and its STMGEN subroutine to insure that the mode 1 in its latest form was being ir.plemented properly. After the program was corrected for necessary changes, it was revised into a simplified form with all extraneous statements in STIGEN that remained from the GA version deleter. Finally, the input data for LAP as suppliec by GA was checked for accuracy. The entire process just described that was done in preparation for the model investigation was time-consuming but necessary. Although several mistakes were located and corrected, no major errors were discc'ered that completely invalidated results obtained previously. The major benefit was an added degree of confidence that could be placed in the steam generator model. In the investigation of various model assumptions, the assumption that the gas dynarics are negligible was questioned. The appropriate 
modification to the model was made to include such effects but no results have been obtained at this time.

As a first step in the investigation of the dependence on the weight factors in Chang's model, some of the stozm generator time response results that he had obtained were verified. Although slightly different due to the corrections made, the responses were still affected greatly by the variation of $W_{3}$. The resoonses of main steam temperature (TS4) and helium outlet node temperatcire (TGIB) to a $1^{\circ} \mathrm{F}$ helium inlet step and a $1 \mathrm{lbm} / \mathrm{sec}$ helium flow step are shown in Figures III.2-5. As shown, the asymptotic values of some variations can more than double when $W_{3}$ is increased from 0.45 10 0.55 . (Note: the $G A$ value suggested for use is 0.30 . Some responses for this value are shown later.)

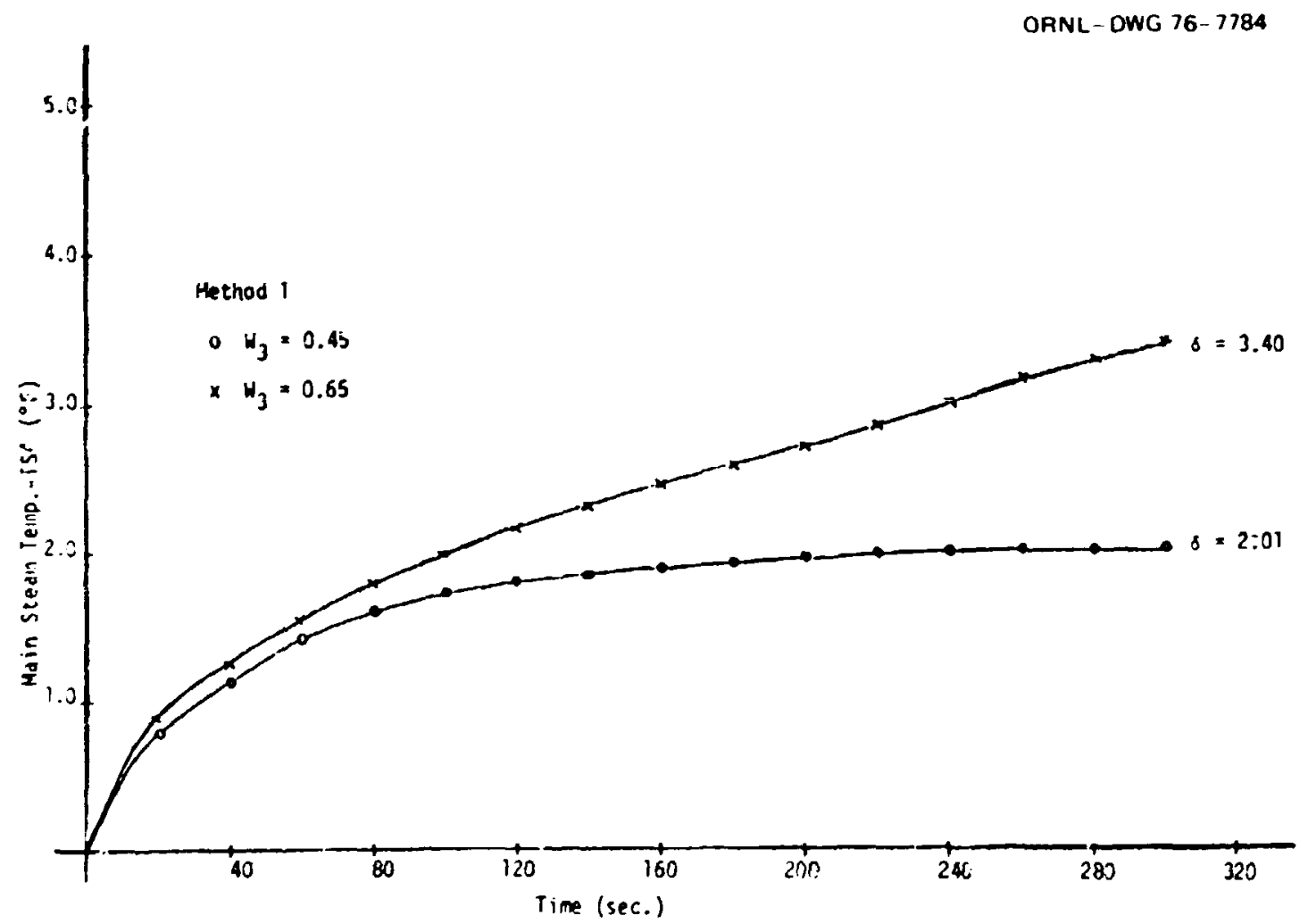

Fig. III.2. Helium Inlet Temperature Perturbation $\left(1^{\circ} \mathrm{F}\right)$. 


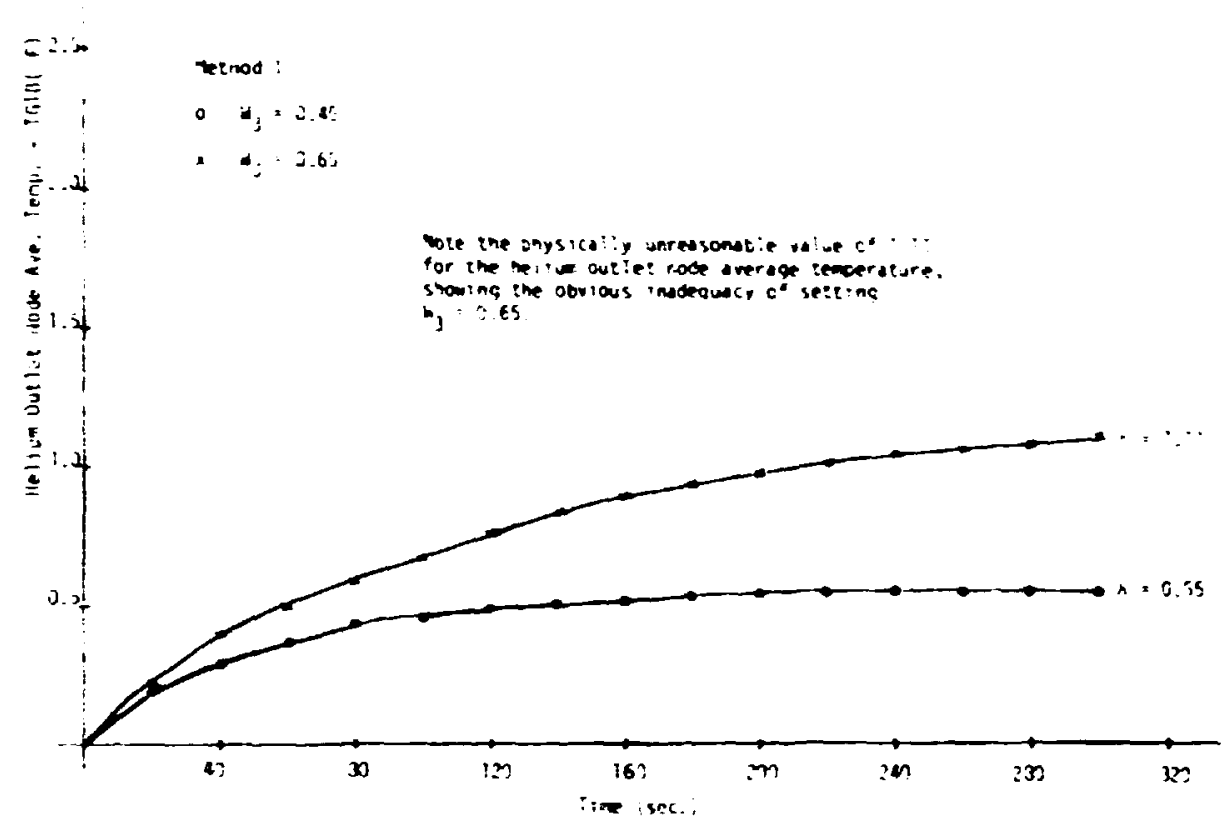

Fig. III.3. Helium Inlet Temperature Perturbation $\left(1^{\circ} \mathrm{F}\right)$.

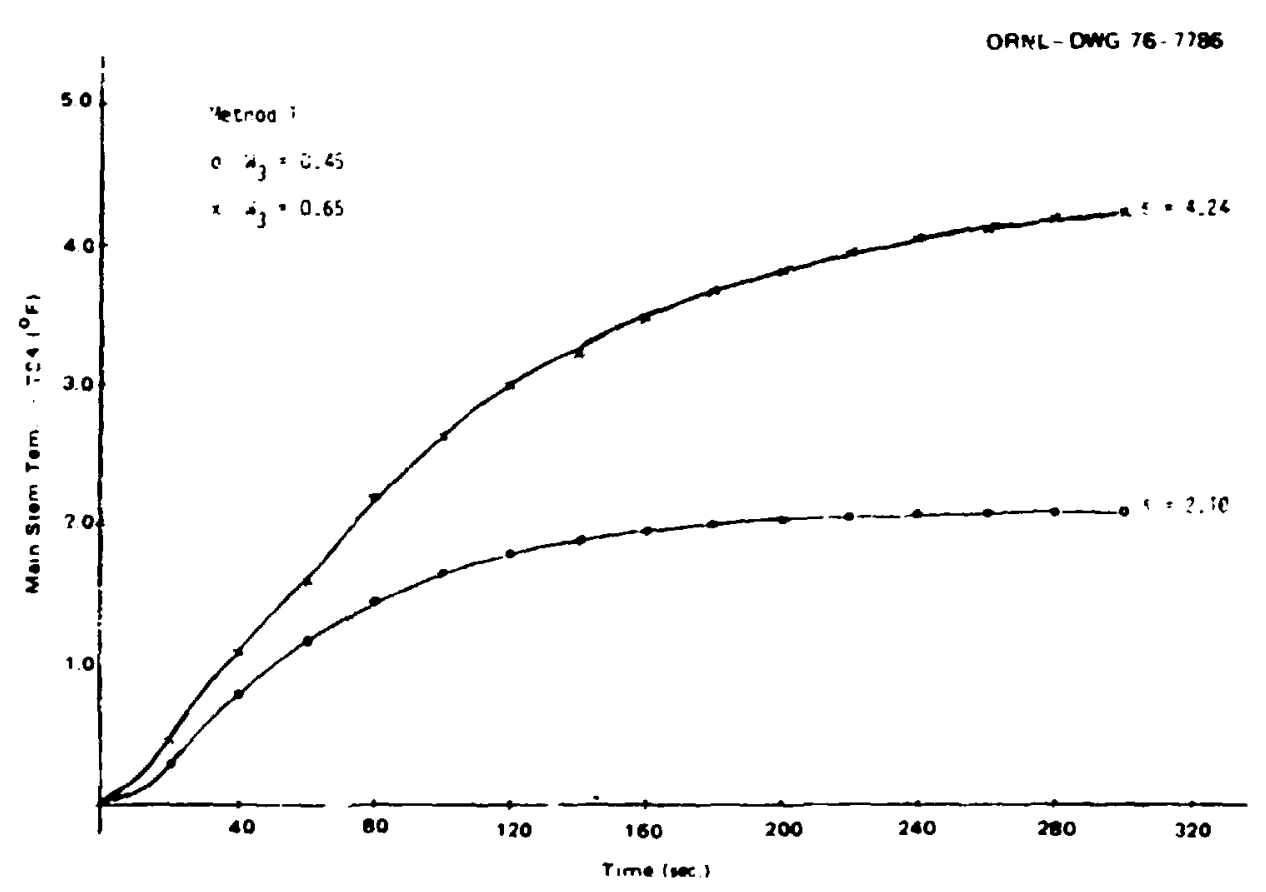

Fig. III.4. Hellum Flow Rate S'.ep (1 $1 \mathrm{bm} / \mathrm{sec})$. 


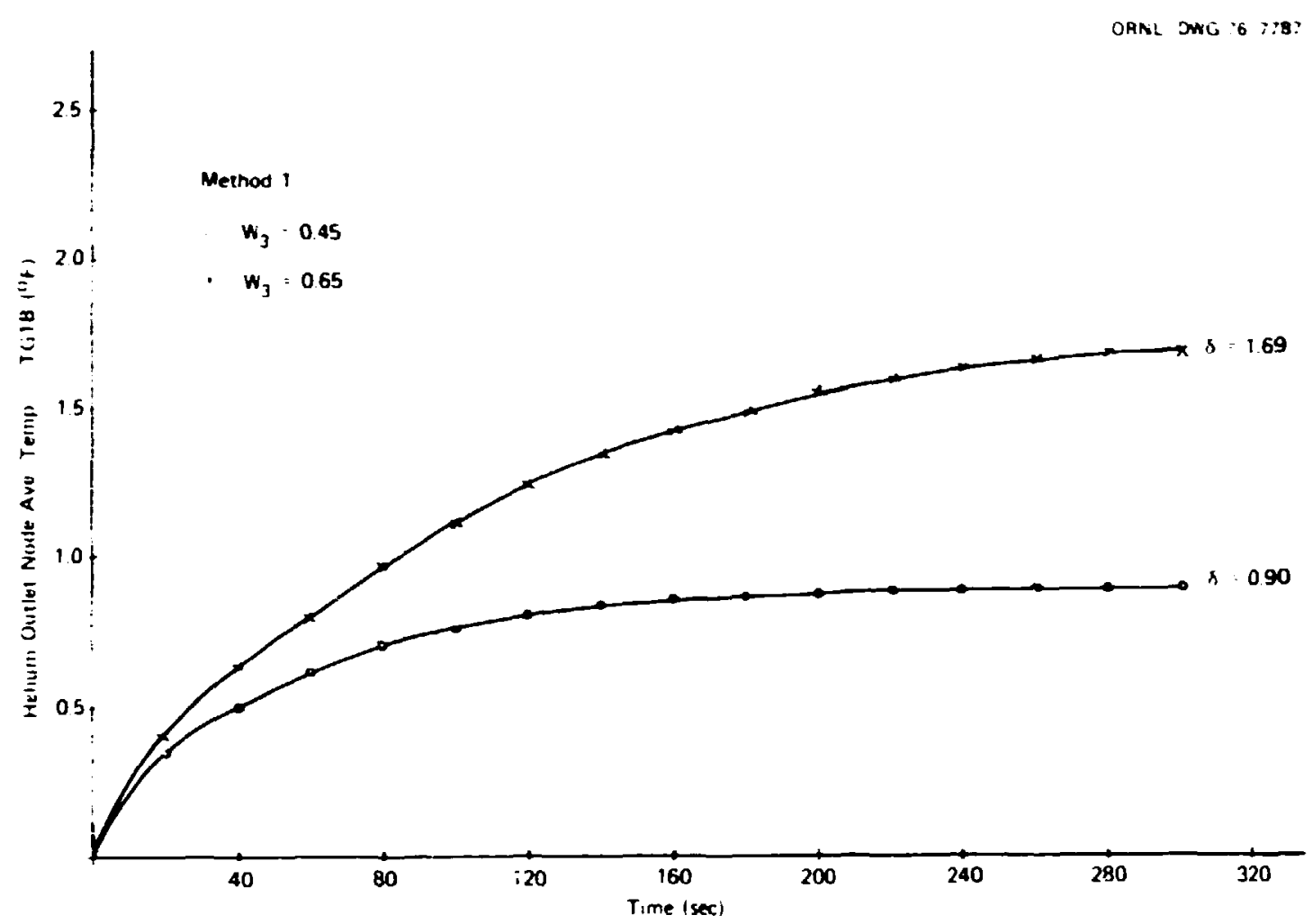

Fig. ill.5. Helium Flow Rate Step (1 $1 \mathrm{bm} / \mathrm{sec})$.

This behavior led to an examination of tine equations and the program for an explanation of this dependence. Included in the input data, GA supplied steady-state values for the helium node boundary temperature $\left(T G_{j}\right)$ but supplied no values for the helium node-averaged temperatures $\left(\overline{T G}_{i}\right)$. These were calculated in the subroutine by Chang using whatever weigh: factors the user chose tc input. This meant that steady state va? ues of $\overline{T G}_{j}$ were being varied as the weights were varied. This change affected the heat transferred out of the primary side as well as other tarms where $\overline{T G}_{i s s}$ appeared in the equation coefficients. So it seemed that the variation of $\overline{T G}_{i s s}$ with weight was responsible for the large dependence on weight factors. 
To avoid this proùien, it ias necessary to caliculate steady-state values of $\overline{i G}_{j}$ that were not weight-dependent. This was accomplished by calculating $\overline{T G}_{\text {iss }}$ with the original GA weight factors. In this way, the steady-state $: a l u e s$ of $\overline{T G}_{j}$ and $T_{i}$ were constant and the weights could be varied independently. Results using this approach are shown in Figures III.6-9. As shown, the time responses were no longer very dependent on weighting factors. This method seemed adequate until the question was raised that variation of weight factors while $\overline{T G}_{i s s}$ and $T G_{\text {iss }}$ were fixed violated the relation in Equation (III.1). To avoid this, it it was decided to calculate $\overline{\mathrm{TG}}_{\text {iss }}$ with the original weights as before but to recalculate $T_{\text {iss }}$ with each new set of weight factors input.

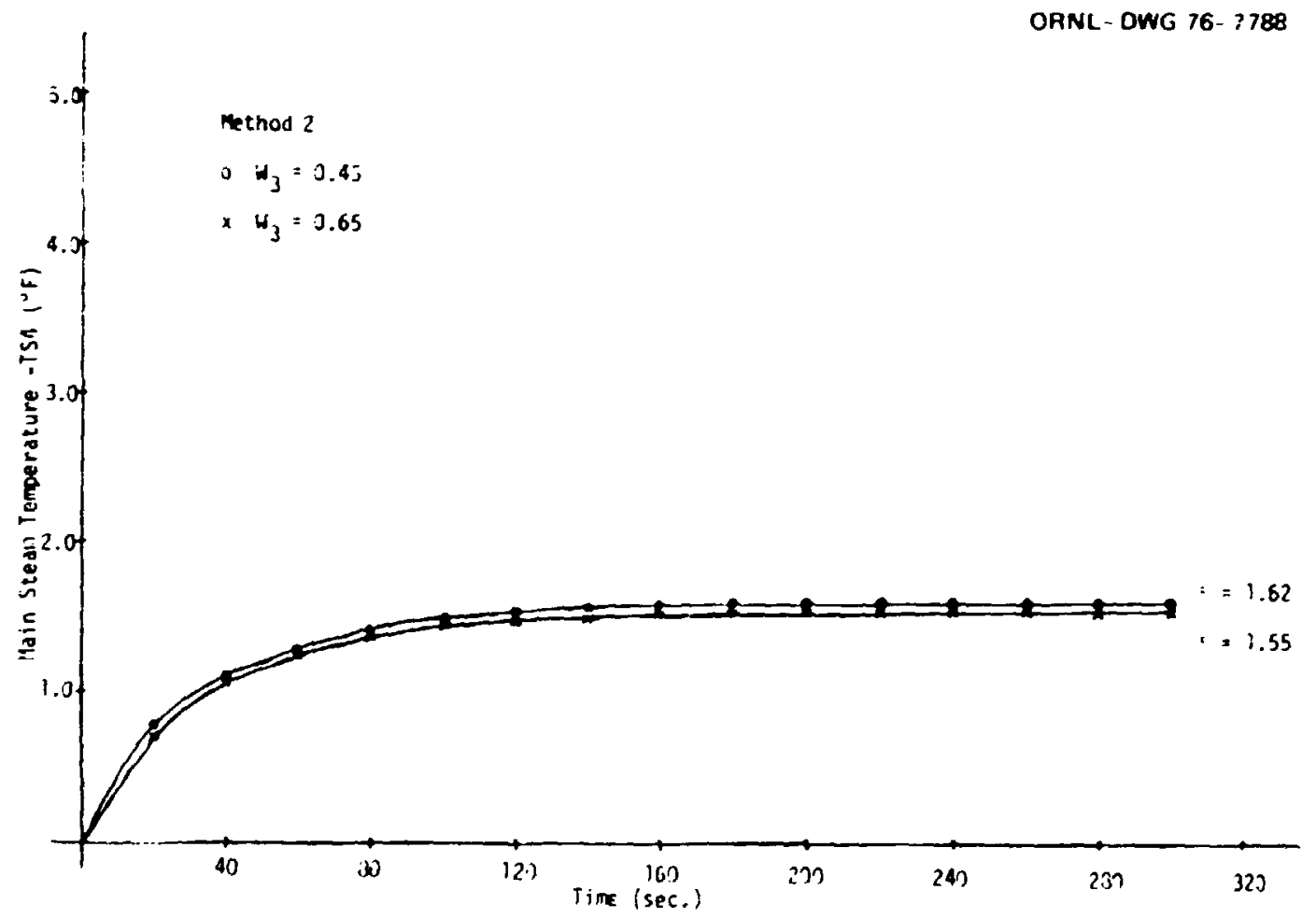

Fig. Ill.6. Helfum Inlet Temperature Perturbation $\left(1^{\circ} \mathrm{F}\right)$. 


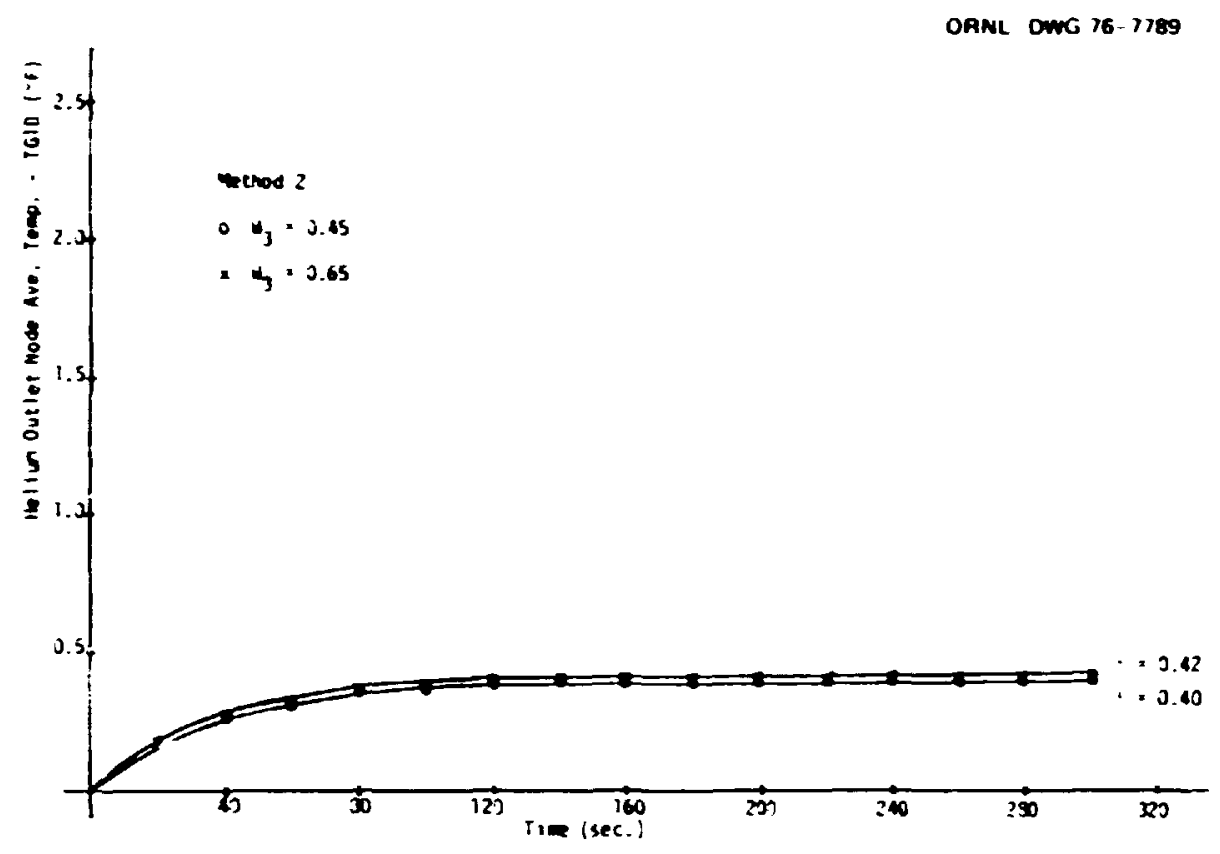

Fig. III.7. Hilium Inle: Temperature Perturbation $\left(1^{\circ} \mathrm{F}\right)$.

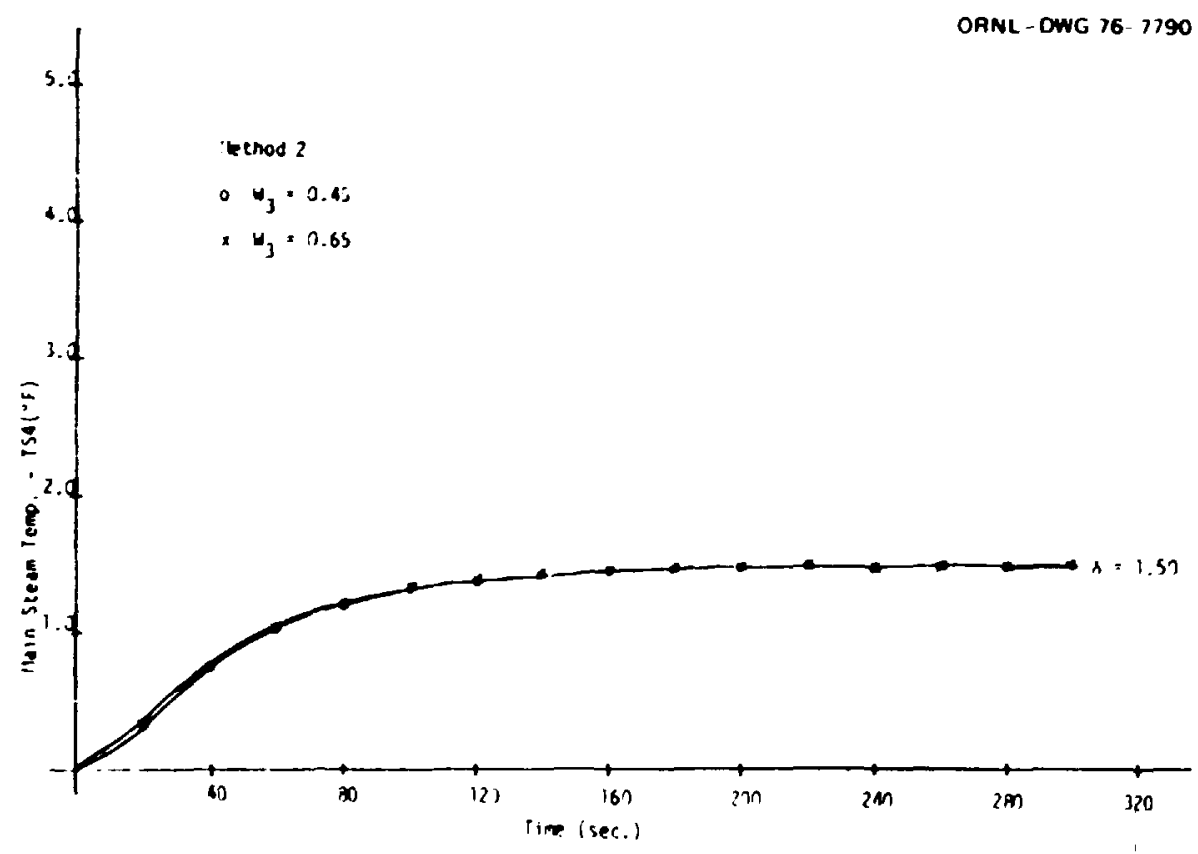

Fig. III.8. Helium Flow Rate Step (1 $1 \mathrm{bm} / \mathrm{sec})$. 


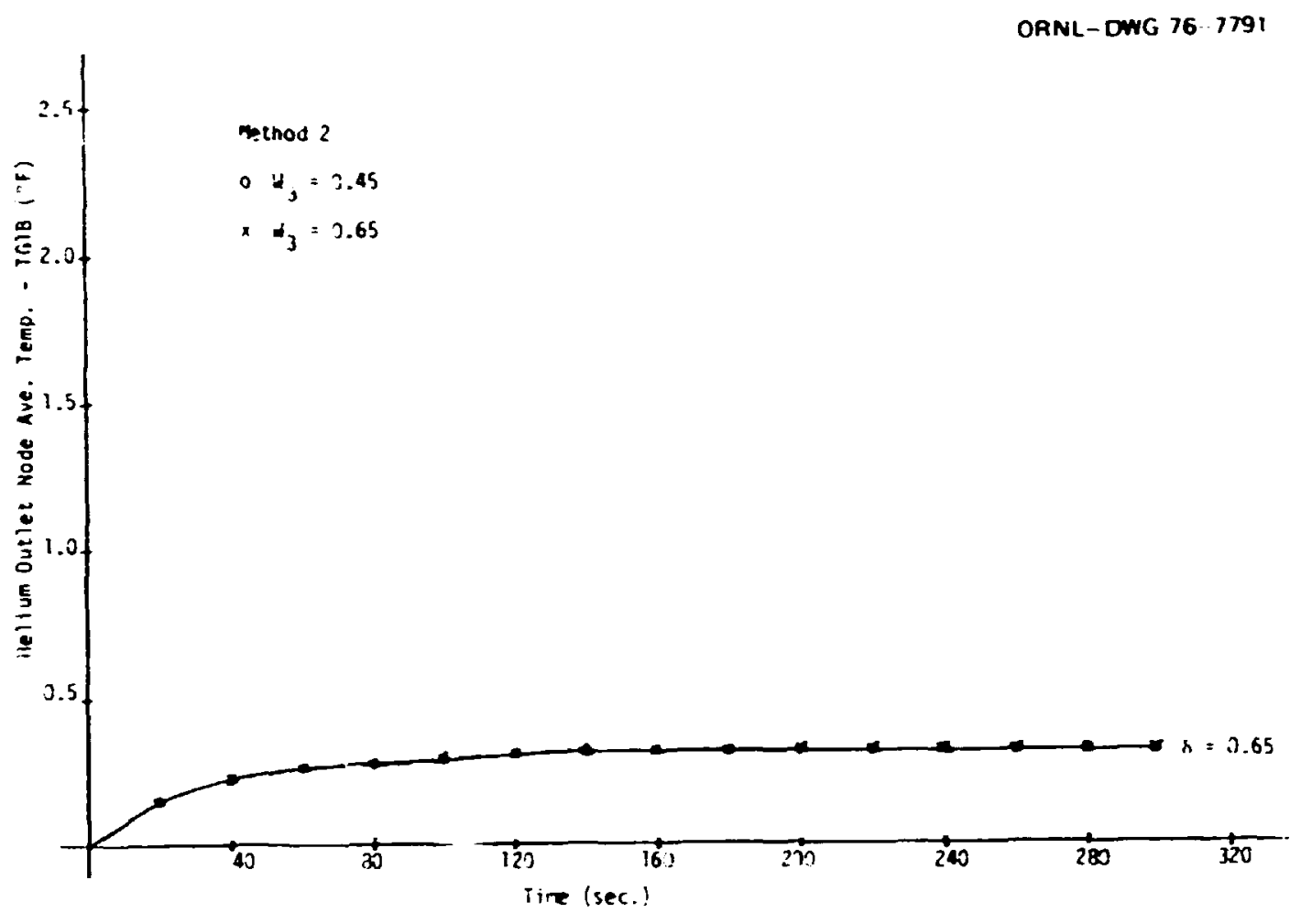

Fig. III.9. Helium Flow Rate Step (1 $\mathrm{lbm} / \mathrm{sec})$.

Results using this method are shown in Figures III.10-13. As shown, the responses are more weight-dependent than those of the second method (Figures III.6-9) Dut less weight-dependent than those of the first method (Figures III.2-5). The third method is actually more closely related to the first since weight changes lead to changes in various steady-state parameters in both schemes. As a result, the flaws inherent in the first method due to steady-state values being adjusted are present in the third as well. However, from the results, the effect of changing $T G_{i s s}$ is not as important as changing $\overline{T G}_{i s s .}$ The previous discussion of weighting schemes illustrates flaws in all three methods. However, when dealing with a linear model, several 


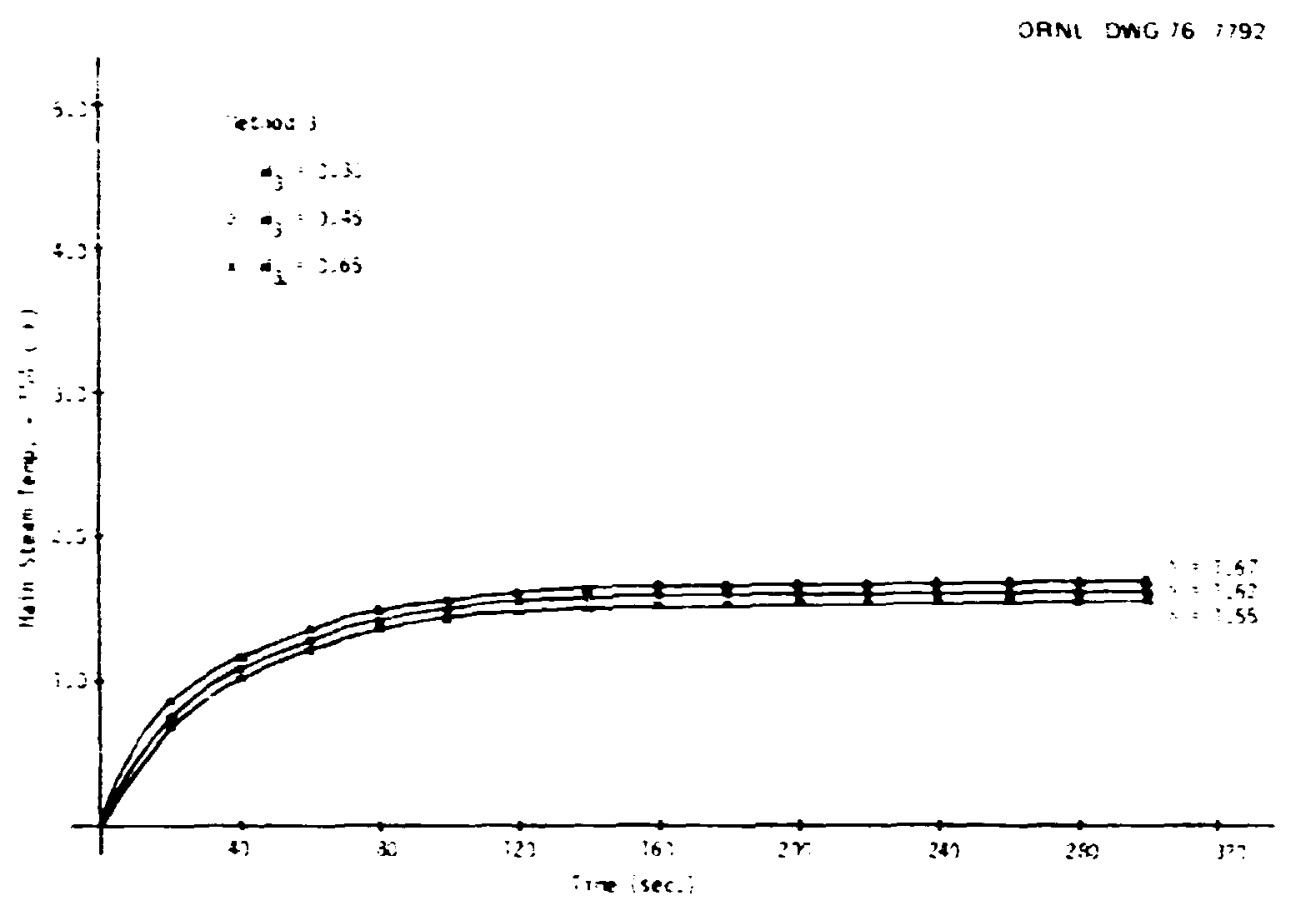

Fig. III.10. Helium Inlet Temperature Perturbation $\left(1^{\circ} \mathrm{F}\right)$.

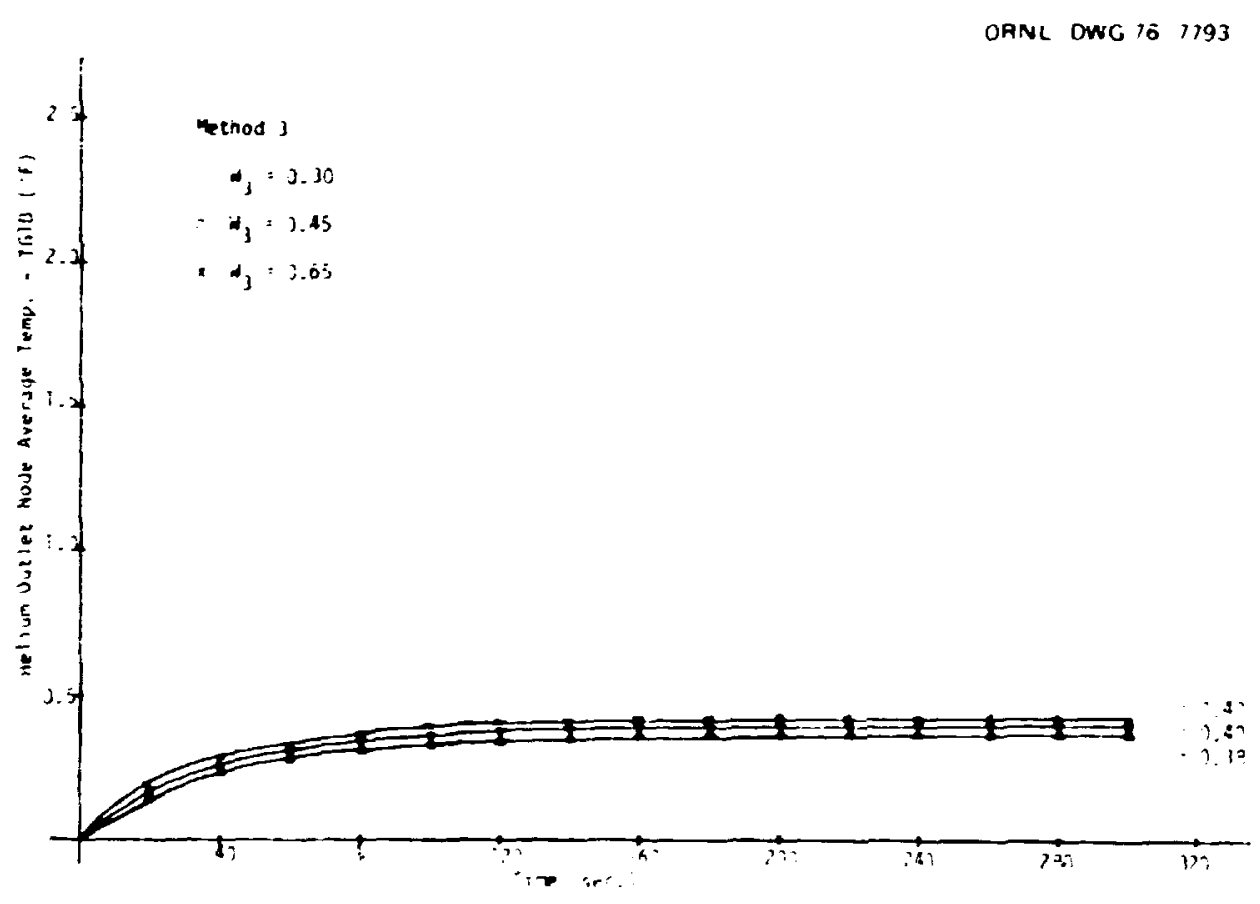

Fig. II i.ll, Helium Inlet Temperature Perturbation $\left(L^{\circ} \mathrm{F}\right)$. 


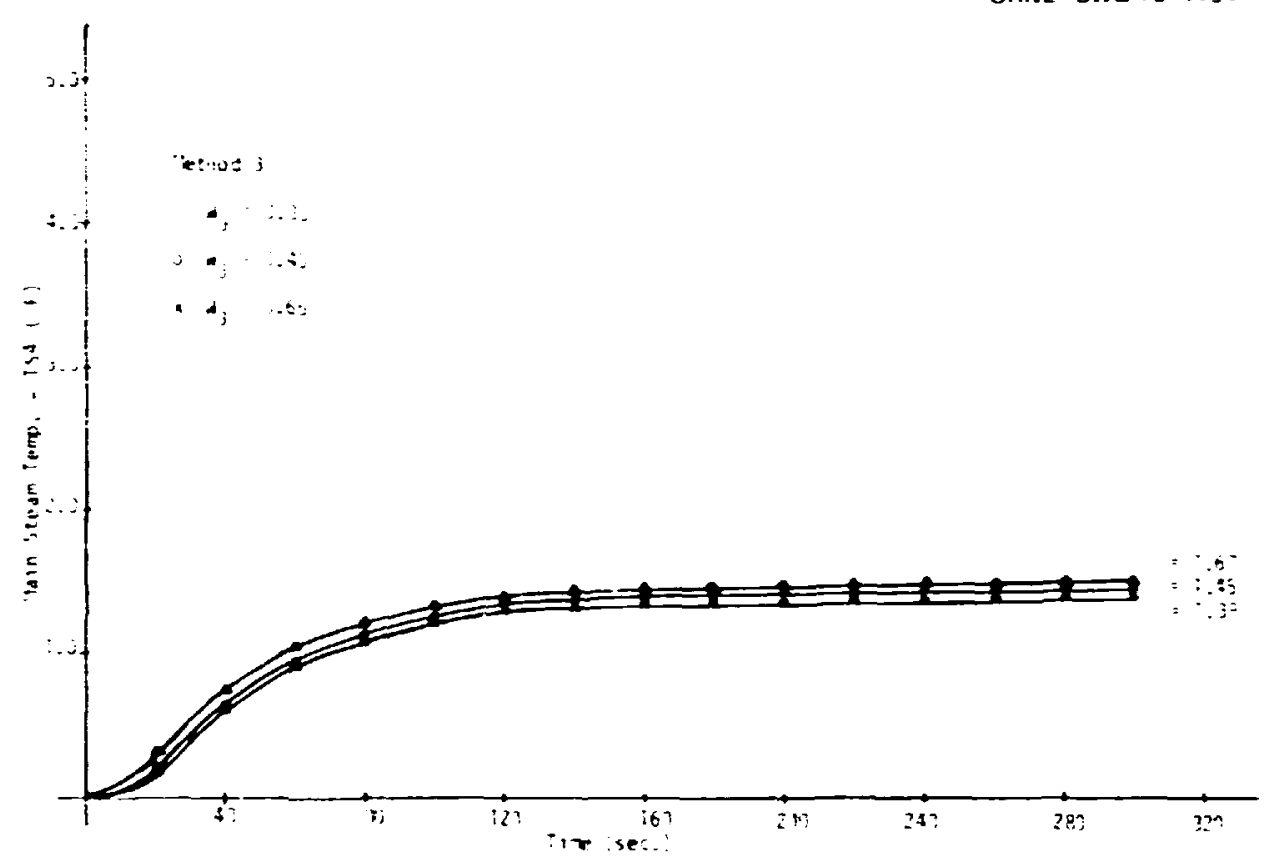

Fig. III.12. Helium Flow Rate Step (1 $1 \mathrm{bm} / \mathrm{sec})$.

ORNL-OWG 76-7795

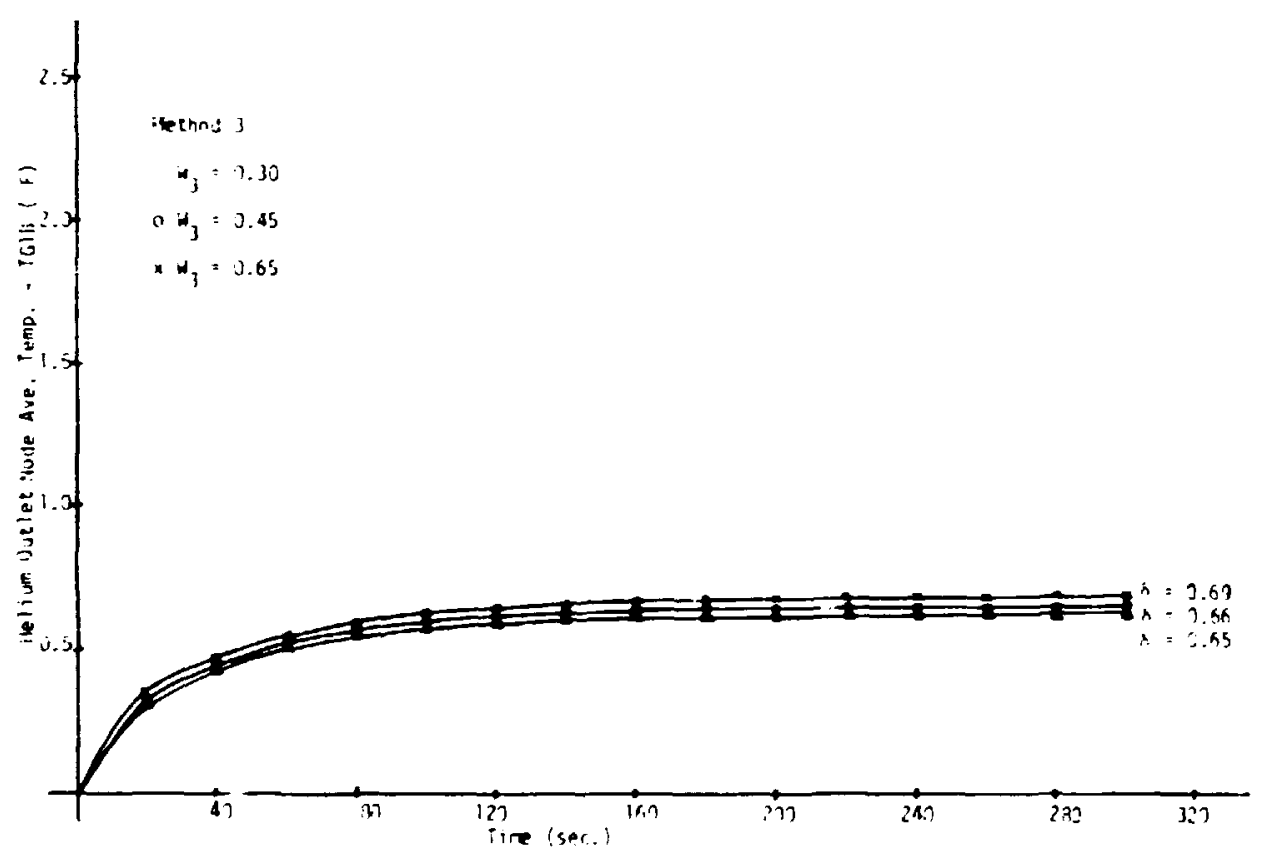

Fig. III.13. Helium Flow Rate Step (1 $1 \mathrm{bm} / \mathrm{sec})$. 
approximations and assumptions have been made already. The question of importance is what additional approximations or assumptions are implied when weighting factors or any other parameters are adjusted. For example, the methods given above modify the model in ways ranging from the amount of heat transferred from the primary side into the tube metal (riethod 1) to the helium boundary outlet temperature (method 3).

The next step in the investigation will be to classify the possible weighting factor schemes along with the assumptions they imply and the results they produce. In addition, methods need to be developed for the variation of the weighting factors on the secondary side independently from the primary. Examination of the relationship between primary and secondary sides throi , the heat balance should produce these methods. If such parameters as pressure and enthalpy on the secondary side could possibly be weighted independsntly of each other, another weighting factor effect could be observed. All of these results have led to a íroadening of the scope of the work in the project to a consideration of the variation of other parameters besides the weight factors. A more general sensitivity study will be performed where various parameters that are fairly arbitrary (like weight factors) or not precisely known (like heat transfer coefficients) can be examined and the accompanying assumptions and consequences outlined.

Since two of the techniques discussed for variation of the primary weight factors involved changing steady-state parameters, possibly the most accurate way to determine the effects of such variations would be to recalculate a completely new set of steady-state values for each 
proposed variation. This method will $b ?$ investigated to see if it is feasible to develop such a capability at UT.

In conclusion, results from the investigation of the effects of variation of weighting factors on the steam generator response have been obtained and have led to a broader sensitivity study of the model. A more general classification of the assumptions made and the results obtained when parameters are varied is desirable and will be developed. Assumptions such as the neglect of gas dynamics have been selected for further verification. These future studies should yield valuable information about significant parameters and assumptions used in this steam generator model. 
CHAPTER IV

NOHLIPEAR, NOOAL SYSTEM MODEL

\section{J. G. Thakkar}

This section briefly describes work being done in formulating the nonlinear system models, for the Fort St. Vrain nuclear steam supply system. The helium circulator model has been completed and some improvenents were made in the reactor, steam generator and reheater models.

\section{IV.1 Steam Properties}

ASMC steam tables were added in the steam generator program using available function generating capability, in CSMP III, of the forms $y=f(x)$ and $y=f(x, z)$. This is a convenient way to calculate thermodynamic properties of both saturated and superheater steani. The results were found to be comparatle with the previous model where stean properties were calculated using polynomial fits. The comparison of two cases for the change in main steam outiet temperature (for $10 \mathrm{r}$ step cirange in ilelium inlet temperature) is shown in Figure [V.I.

\section{IV.2 Reactor}

The reactor model was reformulated using the prompt jump model (1) i.e., setting

$$
\frac{d P}{d t}=\frac{-}{\therefore} P+\because i_{i} C_{i}=0
$$

This gives an algebraic equation for the reactor power, wich allows us to take larger time steps while integrating the heat transfor equations. For small reactivity perturbations $(-56)$ results from the 
- Reference Case (Polynowial fit)

* ASTe stean Iables

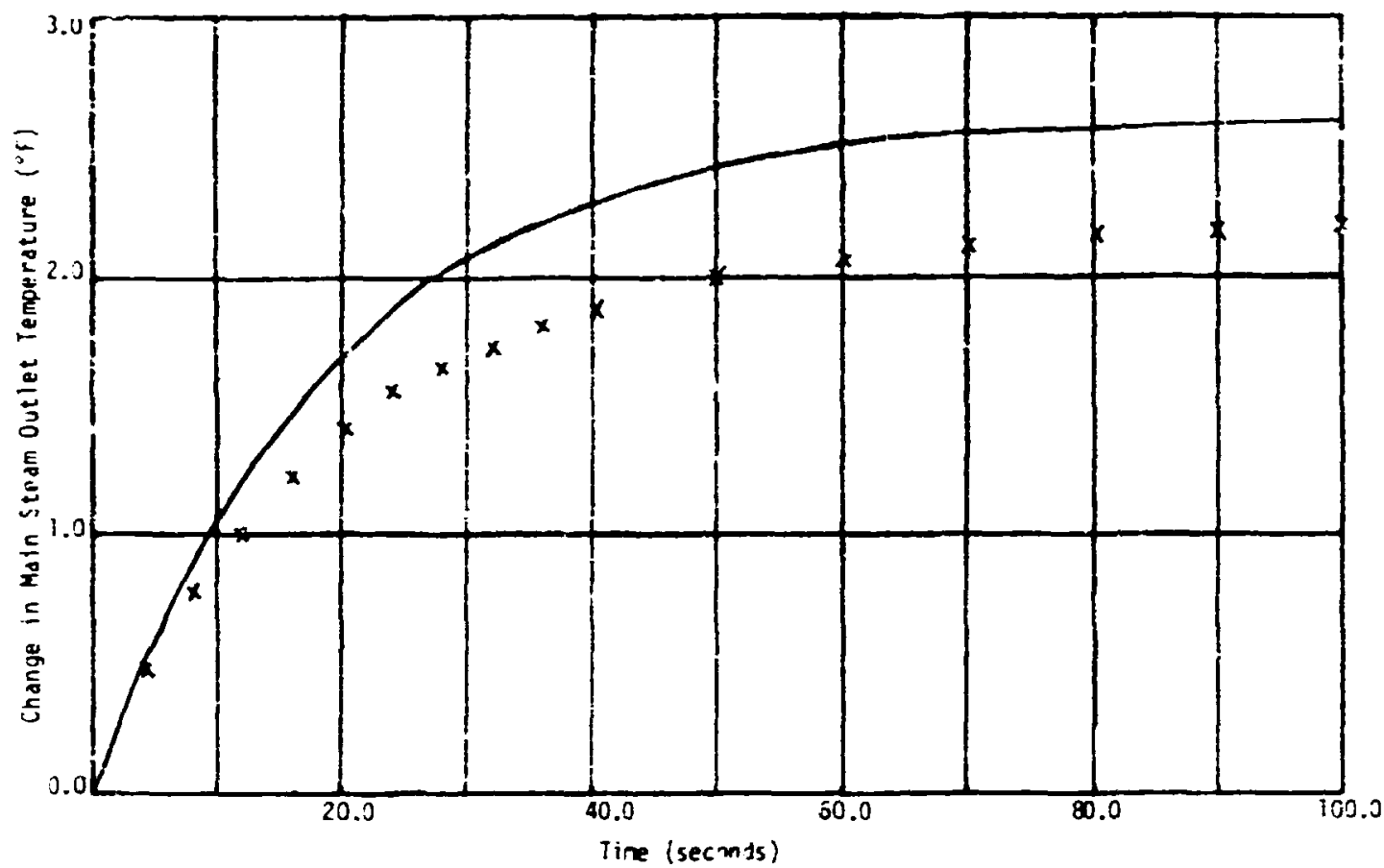

in. IV.I. $1^{\circ} \mathrm{F}$ Step Change in Helium Inlet Temperature.

prompt jump model give less than 2 ; error compared with the reference case. For larger reactivity perturbations $(-406)$ the error increases to about $20 \%$. This is probably due tc the comparatively large reutron life time $\left(-1.85 \times 10^{-4} \mathrm{sec}\right)$ in the HTGR. (The prompt jump model gives accurate results for shorter neutron life times, i.e., in fast reactors $\left.{ }^{(1)}\right)$.

\section{IV.3 Reheater}

The reheater model was updated using new constants and steady state values from the General Atomic memo. (2) This is being tested 
for perturbations in primary inlet temperature, primary flow, and steam flaw. The preliminary results are shown in Figures IV.2-3.

\section{IV.4 Helium Circulator}

Fort St. Vrain has four identical helium circulators, two in each 1oop. Each circulator unit consists of an axial flow compressor, a single stage steam-turbine main drive, and a single stage water-turbine auxillary drive. The schematic of the system is shown in Figure IV,4.

The circulator steam turbine operates on cold reheat steam from the exhaust of the high pressure turbine. The circuiator turbines are in series with the main turbine. Steam enters the blower turbine at about 875 psia and $748^{\circ} \mathrm{F}$ and leaves it at 685 psia and $671^{\circ} \mathrm{F}$. Steam from the exhaust of the circulator turvine flows to the reheater section of the steam generator, About $5 \%$ of trie steam flow bypusses the circulator turbine, at full load, to provide a control margin. The bypass flow is adjusted to the desired value by maintaining a nearly constant pressure ratio across the blower turbine.

Helium from the steam generator exit enters the circulator at about $638^{\circ} \mathrm{F}$ and $686 \mathrm{psia}$ and leaves the circulator at $647^{\circ} \mathrm{F}$ and 700 psia and enters the reactor inlet plenum.

The mathematical model of the circulator is based on conservation equations and performance maps for the turbine and the circulator.

The circulator dynamic characteristics are evaluated finm the conservation of momentum equatic 7

$$
I \frac{d w}{d t}=T_{t}-T_{c}
$$


$\vdots$
$\vdots$
0
$\vdots$
$\vdots$
$\vec{z}$
$\frac{x}{3}$

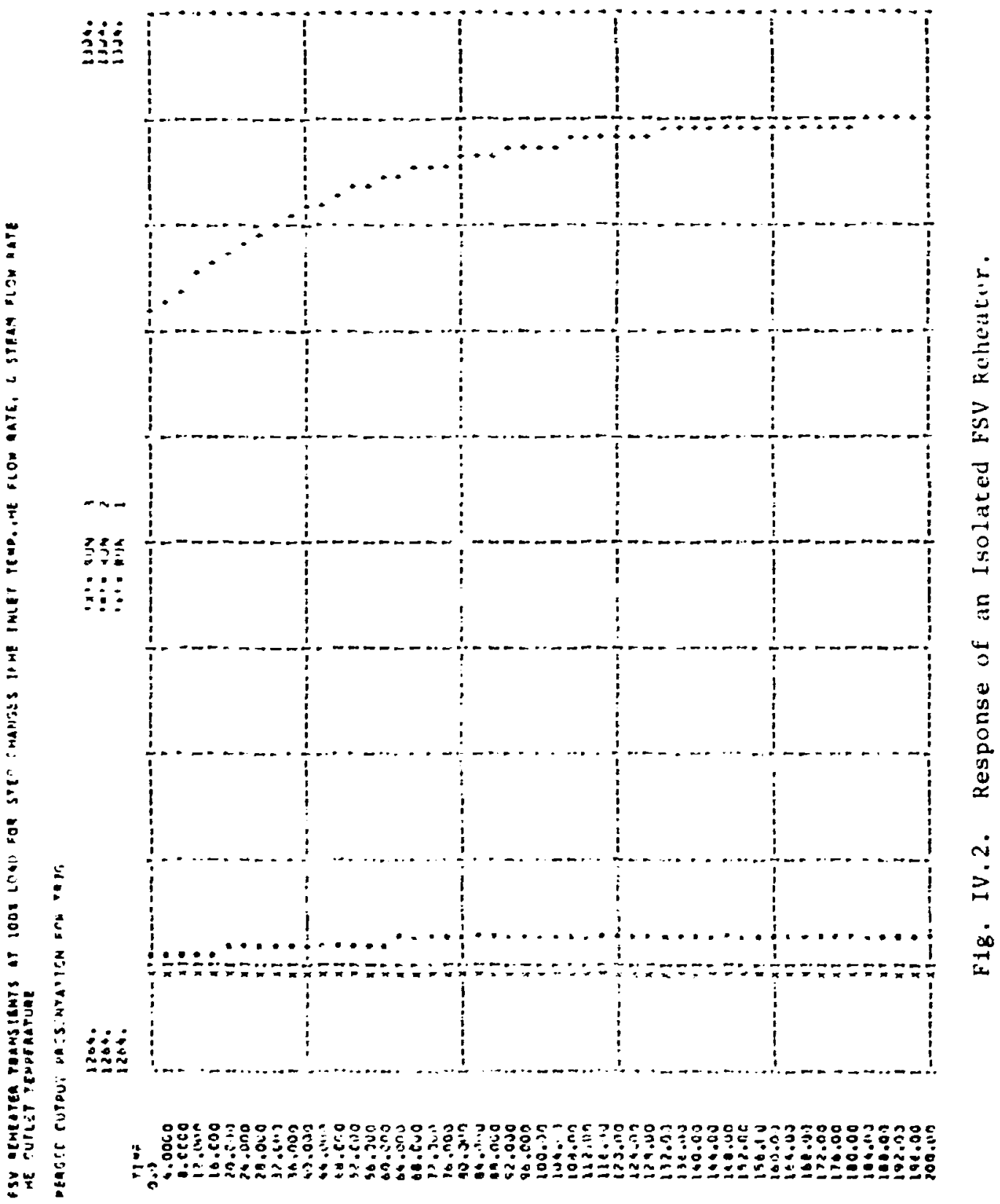




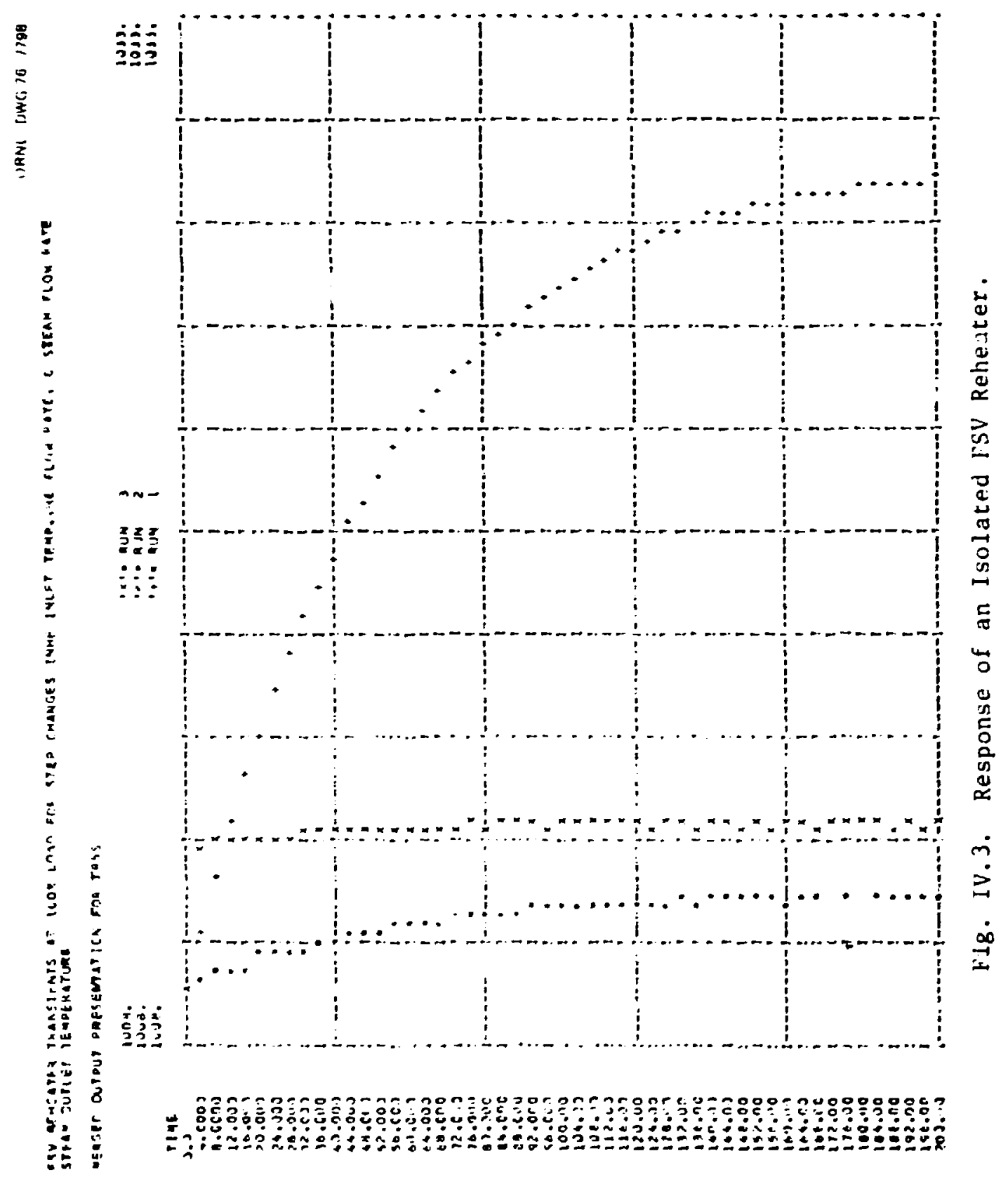


OHNL - OWG 76-7799

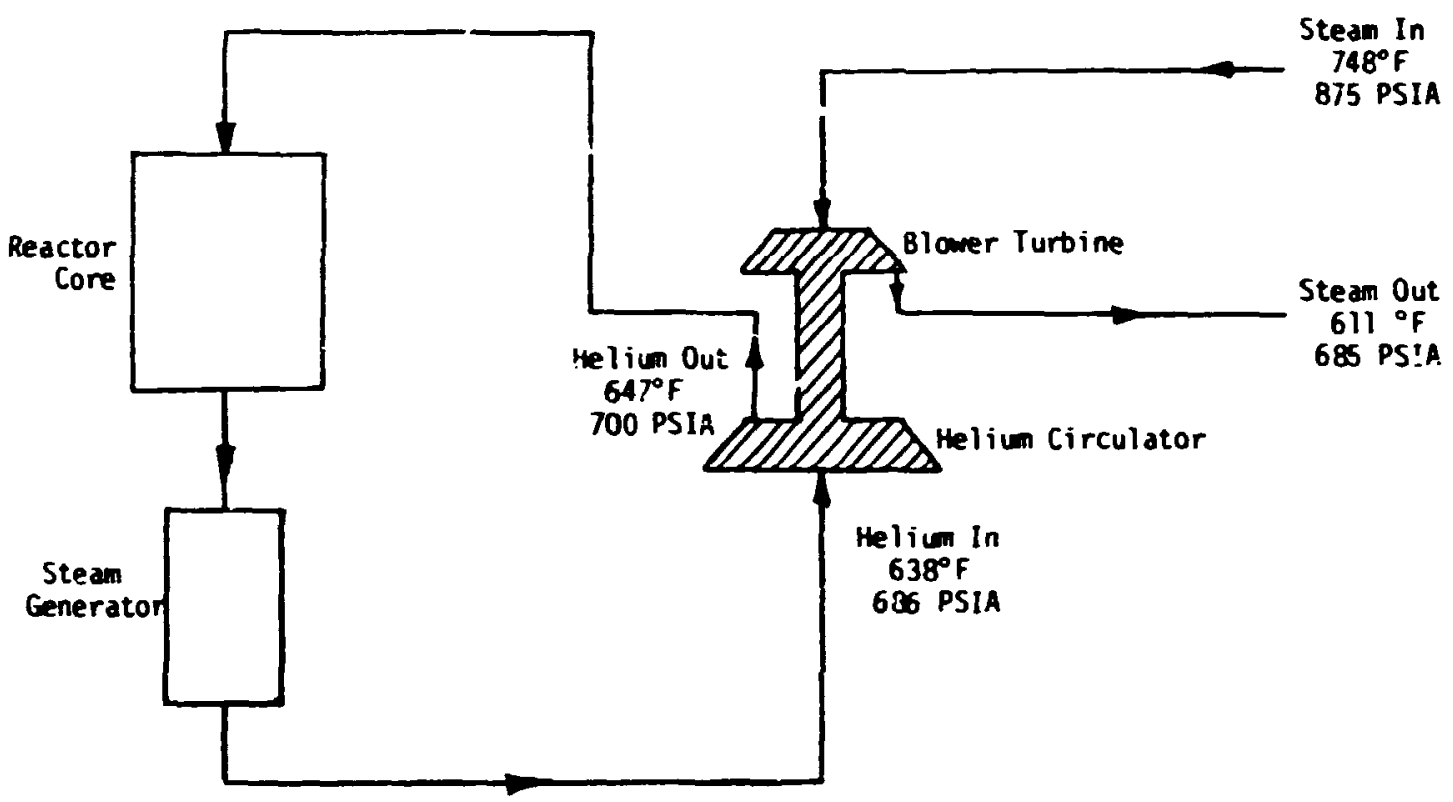

Fig. IV.4. Schematic of Blower Turbine Helium Circulator.

$I$ = mass moment of inertia of the blower turbine rotor shaft

$w=$ angular velocity

$T_{t}=$ Torque exerted by steam

$T_{c}=$ Torque exerted by helium.

$T_{t}$ and $T_{c}$ are calculated from energy balances, as follows

$T_{t}=P_{t} / w$

$T_{c}=P_{c} / w$

$P_{t}=$ power transmitted to the turbine

$P_{C}=$ power required by the compressor.

$$
p_{t}=w_{\text {steam }} \times \Delta_{h s} \times n_{T}
$$




$$
\begin{aligned}
& w_{\text {steam }}=\text { steam flow rate through the turbine } \\
& i_{\text {hs }}=\text { isentropic steam enthalpy drop through the turbine } \\
& { }^{n} T \text { = turbine efficiency } \\
& P_{\mathrm{C}}=w_{\mathrm{He}}{ }^{\star \Delta h} \mathrm{He}^{/ n} \mathrm{c} \\
& w_{\text {He }}=\text { Helium flow rate through the compressor } \\
& \mathrm{ih}_{\mathrm{He}}=\text { Helium enthalpy rise through the compressor } \\
& \eta_{c}=\text { compressor efficiency. } \\
& { }^{7} \mathrm{C},{ }^{\mathrm{T}} \mathrm{T}, \dot{\mathrm{h}}_{\mathrm{hs}} \text {, and } \Delta \mathrm{P}_{\mathrm{He}} \text { are calculated from the compressor and the }
\end{aligned}
$$

turbine performance maps.

The computer program for the circulator model has been completed and is being debugged and tested for the steam flow perturbation.

\section{Future Hork}

1. Model the steam turbines

2. Review the steam generator model, to explain the differences with the linear steam generator model currently used in LAP, particularly for the primary inlet temperature perturbation.

3. Improve pressure drop and heat transfer correlations in the models.

4. Couple all subsystems of Fort St. Vrain to obtain an overall model for the plant and test it for different perturbations and sensitivity to changes in different system parameters.

5. Prepare for correlation of model predictions with trip test results. 
References

1. Saphier, D., L. W. Kirsch, etc., "A Reactor Core Model for a Fast Breeder Power Plant Simulazion," 2nd Power Plant Dynamics, Control and Testing Symposium, Knoxville (1975).

2. Tang, C. K., Fort St. Vrain Linear Anai,sis Program Documentation with Data for $100 \%$ and 25\% Loads, General Atomic Memo SAB:

CKT: 545: 75 (November 1974).

3. Versteegen, P. L., and D. A. Sargis, A Program to Evaluate the Series Steam Turbine Helium Circulator Performance Under Dynamic Conditions, Trpical Report SAI-75-562-LJ (April 1975). 